\title{
WestVirginiaUniversity
}

THE RESEARCH REPOSITORY @ WVU

Graduate Theses, Dissertations, and Problem Reports

2014

\section{A Study of Dynamical and Emission Variabilities in Pulsars}

Andrew D. Seymour

West Virginia University

Follow this and additional works at: https://researchrepository.wvu.edu/etd

\section{Recommended Citation}

Seymour, Andrew D., "A Study of Dynamical and Emission Variabilities in Pulsars" (2014). Graduate Theses, Dissertations, and Problem Reports. 390.

https://researchrepository.wvu.edu/etd/390

This Dissertation is protected by copyright and/or related rights. It has been brought to you by the The Research Repository @ WVU with permission from the rights-holder(s). You are free to use this Dissertation in any way that is permitted by the copyright and related rights legislation that applies to your use. For other uses you must obtain permission from the rights-holder(s) directly, unless additional rights are indicated by a Creative Commons license in the record and/ or on the work itself. This Dissertation has been accepted for inclusion in WVU Graduate Theses, Dissertations, and Problem Reports collection by an authorized administrator of The Research Repository @ WVU.

For more information, please contact researchrepository@mail.wvu.edu. 


\title{
A Study of Dynamical and Emission Variabilities in Pulsars
}

\author{
Andrew D. Seymour \\ Dissertation submitted \\ to the Eberly College of Arts and Sciences \\ at West Virginia University \\ in partial fulfillment of the requirements for the degree of \\ Doctor of Philosophy in \\ Physics \\ Dr. Duncan Lorimer, Ph.D., Chair \\ Dr. Paul Cassak, Ph.D. \\ Dr. John Kuhlman, Ph.D. \\ Dr. Maura McLaughlin, Ph.D. \\ Dr. D.J. Pisano, Ph.D. \\ Department of Physics and Astronomy \\ Morgantown, West Virginia \\ 2014
}

Keywords: Pulsars; Neutron Stars; Chaos Theory

Copyright 2014 Andrew D. Seymour 


\title{
ABSTRACT
}

\section{A Study of Dynamical and Emission Variabilities in Pulsars}

\author{
Andrew D. Seymour
}

Pulsars are rapidly rotating highly magnetized neutron stars thought to have been formed in the core-collapse supernova of massive stars. Ever since their discovery, pulsars have shown complex behaviors. This is certainly true for their emission mechanism, which is still not fully understood. This is primarily because of the abrupt changes that appear in the pulse profile. Recent discoveries have shown that these emission changes effect the spin dynamics, particularly the spin-down rate. This indicates that pulsar emissions are even more complex than previously thought. The goal of this thesis is to apply new analysis techniques to help shed light on the pulsar emission problem.

Over the past decade, it has become apparent that a class of 'bursting pulsars' exist with the discovery of PSR J1752+2359 and PSR J1938+2213. In these pulsars, a sharp increase in the emission intensity is observed that then tends to systematically drop-off from pulse-to-pulse. We describe the discovery of such a relationship in high-sensitivity observations of the young (characteristic age of 90,000 yrs) $0.33 \mathrm{~s}$ pulsar B0611+22 at both $327 \mathrm{MHz}$ and $1400 \mathrm{MHz}$ with the Arecibo observatory. While it was previously shown that B0611+22 has mode-switching properties, the data presented here show that this pulsar emits bursts with characteristic time-scales of several hundred seconds. At $327 \mathrm{MHz}$, the pulsar shows steady behavior in one emission mode which is enhanced by bursting emission slightly offset in pulse phase from this steady emission. Contrastingly at $1400 \mathrm{MHz}$, the two modes appear to behave in a competing operation while still offset in phase. Using a fluctuation spectrum analysis, we also investigate each mode independently for sub-pulse drifting. Neither emission mode (i.e. during bursts or persistent emission) shows the presence of the drifting sub-pulse phenomenon. While further examples of this behavior and studies at different wavelengths are required, it appears that this phenomenon may be quite common among the pulsar population.

Until now, PSR J1752+2359 is the only one of the three bursting pulsars that has not been accompanied by a lower energy level normal emission mode. Rather, it has appeared to null between bursting events. We have been able to show through the pulse-energy distribution that PSR J1752+2359 does indeed have a normal mode with a peak flux at $0.17 \pm 0.03 \mathrm{mJy}$ with a pulse energy of $1.0 \pm 0.1 \mu \mathrm{Jy}$-s. It is also shown that PSR J1752+2359 presents no evidence for the drifting sub-pulse phenomenon in either emission mode. This is consistent with what has been seen in PSR B0611+22.

We also present techniques that searched for chaotic behaviors within the spin dynamics of 17 pulsars. These techniques allow us to re-sample the original spin-down 
rate estimates without losing structural information, and to search for evidence of a strange attractor within these frequency derivative time series. We demonstrate the effectiveness of our methods by applying them to a component of the Lorenz and Rössler attractors that were sampled with similar cadence to the pulsar time series. Our measurements of correlation dimension and Lyapunov exponent show that the underlying behavior appears to be driven by a strange attractor with approximately three governing non-linear differential equations. This is particularly apparent in the case of PSR B1828-11 where a correlation dimension of $2.06 \pm 0.03$ and a Lyapunov exponent of $(4.0 \pm 0.3) \times 10^{-4}$ inverse days were measured. These results provide an additional diagnostic for testing future models of this behavior.

Lastly, we introduce future plans to further improve our understanding of the bursting phenomena and spin-down rate changes seen here. Simultaneous observations at different frequencies will help determine how a burst is propagating through different emission regions. Along with this, a recent analogous discovery to bursting implies that bursting events may be accompanied by X-ray changes. Scheduled X-ray observations will soon determine if this is true. As data sets cover even larger amounts of time, the non-linear analysis will improve and can be utilized to test theoretical models. We also present data provided by Andrew Lyne that hints at a connection between bursting and spin-down changes. 


\section{Dedication}

The author wishes to dedicate this thesis to the memory of life long family friend Gerald P. Lough.

A truck driver by profession that knew of the author's

desire to travel and told him at an influential age,

"Keep doing good in school, because those books

will take you farther than my truck ever will..." 


\section{Acknowledgments}

Any endeavor is never truly done alone and as this endeavor comes to a close, I wish to acknowledge those who have made this possible and who have greatly influenced me to embark on such a task.

I would first like to thank my father, David Seymour. Simply, I would not be the person I am today without his constant support. I only began to see how science could be fun and exciting, when he help me built a simple hovercraft for a junior high science fair. This eventually steered me towards mechanical and aerospace engineering.

When finishing my degrees in engineering, I decided to minor in physics. There I rediscovered my excitement for science, mainly due to the wonderful teaching style of Dr. Martin Ferer. Because our great talks in his paper stacked office, I wanted to continue with a career in physics. I would then have to thank Dr. Earl Scime for giving me this opportunity by allowing me to enter the physics graduate program at West Virginia University.

I next want to acknowledge my $\mathrm{PhD}$ advisor Dr. Duncan Lorimer. Later in my graduate career, after a couple unsuccessful attempts to join a research group, he approached me to work with him. A decision I have never regretted. Through this work, I have had several fantastic experiences and travels that both Dr. Lorimer and Dr. Maura McLaughin have created.

I would like to particularly thank Dr. McLaughin for the spirited conversations that we have had. Without her great wisdom and knowledge, this paper would not be at the quality that it is today. I wish to thank my office mates Katie Rabidoux and Spencer Wolfe for helping expand my knowledge of astronomy. I would also like to express my gratitude for the thankless hours of proofreading that Katie has done for this thesis.

I would like to thank all my friends in both panhandles of West Virginia and the Metropolitan pool hall which have helped me relax and kept me grounded throughout my entire college career.

I wish to close, by thanking my loving and caring wife Jee Eun. She has been my rock through most of my graduate career. Her constant happiness and support has been the only thing to get me through to this point and I cannot wait to start the rest of our lives together. 


\section{Table of Contents}

List of Tables $\quad$ vi

List of Figures

1 Pulsars 1

1.1 Their discovery . . . . . . . . . . . . . . . . . . . . 1

1.2 Pulse origins . . . . . . . . . . . . . . . . . . . 3

1.3 Observed emission variations . . . . . . . . . . . . . . . 8

1.3.1 Drifting sub-pulses . . . . . . . . . . . . . . . . 9

1.3 .2 Nulling. . . . . . . . . . . . . . . . . . . 11

1.3.3 Mode switching . . . . . . . . . . . . . . . . . 13

1.3.4 Bursting ........................ 14

1.4 Emission variations accompanied by spin-down fluctuations. . . . . . 15

1.4.1 Basic pulsar spin dynamics . . . . . . . . . . . . . . . . 16

1.4.2 Abrupt spin-down rate variations . . . . . . . . . . . . . . 19

1.4.2.1 Intermittent pulsars . . . . . . . . . . . . . . . . . 19

1.4.2.2 Component appearance and drifting . . . . . . . . 22

1.4.3 Continuous spin-down rate variations . . . . . . . . . . . . . 24

1.5 Chaotic behaviors . . . . . . . . . . . . . . . . 26

1.5.1 Fractal dimensions ................ . . . 31

1.6 Thesis outline . . . . . . . . . . . . . . . . . . . . . . 32

2 Pulse to pulse study of B0611+22 34

2.1 Observations . . . . . . . . . . . . . . . . . 35

2.2 Data analysis . . . . . . . . . . . . . . . . . . . . . . . . . . . . . . . . .

2.2.1 Individual pulse profiles . . . . . . . . . . . . . . . 36

2.2.2 Revealing the emission modes . . . . . . . . . . . . . . 37

2.2.3 Gaussian fitting and skewness measurements . . . . . . . . . . 41

2.2.3.1 Gaussian and skewness results . . . . . . . . . . 41

2.2.3.2 Bursting rate . . . . . . . . . . . . . 45

2.2.4 Pulse energy distributions . . . . . . . . . . . . . . . . . . 46

2.3 Sub-pulse drifting analysis . . . . . . . . . . . . . . . . . . . . . . . . . . . . . . . . . . . . . . .

2.4 Conclusion . . . . . . . . . . . . . . . . . . . 52

3 A search for an underlying normal mode in J1752+2359.

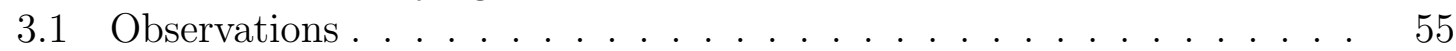

3.2 Pulse energy distribution . . . . . . . . . . . . . . . . . 56

3.3 Positive thinking . . . . . . . . . . . . . . . . . . . 59

3.3.1 Positives in a row .................. 61

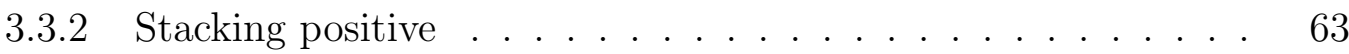

3.4 Sub-pulse drift . . . . . . . . . . . . . . . . . . . 65

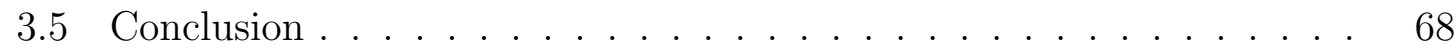


4 Dynamical Study of Pulsar Spin-down Rates 70

4.1 Linear Analysis . . . . . . . . . . . . . . . . . . . 70

4.1.1 Mind the gap . . . . . . . . . . . . . . . . 70

4.1.2 Turning point analysis . . . . . . . . . . . . . . . 71

4.1.3 Cubic spline . . . . . . . . . . . . . . . . . . . 75

4.1.4 Fourier transform . . . . . . . . . . . . . . . . . . 75

4.1.4.1 Noise reduction . . . . . . . . . . . . . 76

4.2 Non-linear Analysis . . . . . . . . . . . . . . . . . . . . . 77

4.2.1 Attractor reconstruction . . . . . . . . . . . . . . . 78

4.2.1.1 Method of delays . . . . . . . . . . . . . 78

4.2.1.2 Time delay . . . . . . . . . . . . . . . . . . 78

4.2.1.3 Correlation dimension ............. . . 81

4.2.1.4 Theiler window . . . . . . . . . . . . . . 85

4.2.1.5 Space time separations . . . . . . . . . . . 85

4.2.1.6 Embedding to higher dimensions . . . . . . . . . . 87

4.2.1.7 Surrogate data . . . . . . . . . . . . . . . . 89

4.2.1.8 Correlation benchmark testing . . . . . . . . . 90

4.2.2 Measuring the butterfly effect . . . . . . . . . . . . . . . . . 93

4.2.2.1 Lyapunov exponent. . . . . . . . . . . . . . . 93

4.2.2.2 Lyapunov Benchmark Testing . . . . . . . . . . . . 97

4.3 Conclusions . . . . . . . . . . . . . . . . . . . . 99

5 Conclusions 102

$\begin{array}{ll}\text { A Skewness equations } & 109\end{array}$

B Turning point variance derivation 111

C Correlation dimension error calculations 114

$\begin{array}{ll}\text { D Chaos figures } & 117\end{array}$ 


\section{List of Tables}

1.1 Effects on the braking index . . . . . . . . . . . . . . . . . . . 19

2.1 Summary of B0611+22 observations . . . . . . . . . . . 35

4.1 Distance from $\mu_{T}$ for 17 pulsars . . . . . . . . . . . . . . . . . 74

4.2 The benchmark results for the correlation dimension for 12 pulsars . . 93

D.1 Fourier amplitudes and low pass time series . . . . . . . . . . 117

D.2 Reconstructed attractors . . . . . . . . . . . . . . . . . . 120

D.3 Correlation Sums and Correlation Dimension Measurements . . . . . 123 


\section{List of Figures}

1.1 First pen recording of CP1919 . . . . . . . . . . . . . . . 2

1.2 The pulsar model . . . . . . . . . . . . . . . . . . . . 4

1.3 The Goldreich-Julian model . . . . . . . . . . . . . . . . . 5

1.4 Drifting sub-pulses example . . . . . . . . . . . . . . . . 10

1.5 Nulling pulse energy histograms . . . . . . . . . . . . . . . . . . 12

1.6 Mode switching example . . . . . . . . . . . . . . . . . . . 14

1.7 Bursting event example . . . . . . . . . . . . . . . . . . . 15

$1.8 \quad P-\dot{P}$ diagram . . . . . . . . . . . . . . . . . . . . 18

1.9 Spin frequency and timing residuals of B1931+24 . . . . . . . . . 20

1.10 Variations in the profile shape and spin-down rate seen in J0738-4042 23

1.11 Spin-down rate variations presented in Lyne et al. (2010) . . . . . . . 25

1.12 'Butterfly effect' example . . . . . . . . . . . . . . . . . . 28

1.13 Three dimensional visualization of two chaotic attractors . . . . . . . 29

1.14 The route to chaos for the Rössler equations . . . . . . . . . . . . . . 30

1.15 Iterations leading to the von Koch curve . . . . . . . . . . . . . . . . 31

$2.1 \quad$ B0611+22 enhanced emission at $327 \mathrm{MHz} \quad \ldots \ldots \ldots$

$2.2 \mathrm{~B} 0611+22$ bursting events at $327 \mathrm{MHz} \ldots \ldots \ldots \ldots$

2.3 B0611+22 undulation at $1.4 \mathrm{GHz} \ldots \ldots$. . . . . . . . . . . . . . . . . 39

2.4 Structure profiles of left and right side of B0611+22 at $1.4 \mathrm{GHz}$. . . 40

2.5 Histograms of Gaussian parameters at $327 \mathrm{MHz}$. . . . . . . . . . . . 42

2.6 Histogram of Gaussian parameters at $1.4 \mathrm{GHz}$. . . . . . . . . . . . . 43

2.7 Histograms of Gaussian parameters with normal mode removed . . . 44

2.8 Pulse energy distributions . . . . . . . . . . . . . . . . . . 46

2.9 Off pulse energy distribution . . . . . . . . . . . . . . . . . . . 48

2.10 The fluctuation spectra for bursting mode at $327 \mathrm{MHz}$. . . . . . . . 49

2.11 The fluctuation spectra for normal mode at $327 \mathrm{MHz}$. . . . . . . . . 50

$3.1 \quad$ J1752+2359 mean profile . . . . . . . . . . . . . . . . . 56

3.2 Energy values for $\mathrm{J} 1752+2359 \ldots \ldots \ldots$. . . . . . . . . . . . . . . . . . . . . . . . 57

3.3 Low energy profile with distribution of the peak flux . . . . . . . 58

3.4 Positive probability profile base on positives in each bin . . . . . . . . 60

3.5 Positive probability profile base on two positives in a row . . . . . . . 62

3.6 Positive probability profile base on summed positive-in-a-row statistics 65

3.7 Fluctuation spectra of J1752+2359 . . . . . . . . . . . . . . . 66

4.1 Example of observational gaps in spin-down rate data . . . . . . . . . 71

4.2 Turning point examples . . . . . . . . . . . . . . . . . . . . . . . . . . . . . 72

4.3 Turning point combinations . . . . . . . . . . . . . . . 73

4.4 The Fourier transform of B1642-03 with low pass time series . . . . 77

4.5 Lorenz attractor XY plane with reconstructed time delayed attractor $\quad 79$

4.6 An ideal example of autocorrelation coefficients . . . . . . . . . . . 80

4.7 The topology of the pulsar time series . . . . . . . . . . . . . . . 82 
4.8 Examples of $\frac{d \ln N_{x}}{d \ln R}$ producing the correct dimension . . . . . . . . . . 83

4.9 Space time separation plot . . . . . . . . . . . . . . 86

$4.10 \ln C$ versus $\ln R$ with correlation dimension measurements for B1828-11 88

4.11 Fourier series with correlation dimension measurement for B1540-06 90

4.12 Benchmark testing for B1828-11 . . . . . . . . . . . . . . 92

$4.13 S(\Delta t)$ versus time elapsed for B1828-11 and a surrogate data set . . 96

4.14 Benchmark results of $S(\Delta t)$ versus time elapsed for B1828-11 . . . . 98

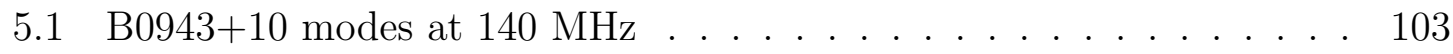

5.2 Spin-down rate changes for B0611+22 . . . . . . . . . . . . . . 104 


\title{
Chapter 1
}

\author{
Pulsars
}

\subsection{Their discovery}

In the mid-1960s, Cambridge Professor Antony Hewish designed a large radio telescope to find variations in radio signals coming from quasars which occurred when these signals traveled through turbulent solar winds in interplanetary space. This telescope spanned over 4.5 acres with over a thousand wooden posts, more than twothousand dipoles, and 120 miles of wire and cable (Bell Burnell, 1977). All of it was assembled by about five university students one summer, one of which was Hewish's Ph.D. student Jocelyn Bell Burnell.

Once operational, the telescope scanned the sky between declinations of $+50^{\circ}$ and $-10^{\circ}$ once every four days (Bell Burnell, 1977). The signals were recorded on four different 3-track pen chart recorders, and between them they produced 96 feet of chart paper every day. These charts were analyzed solely by hand by Jocelyn Bell, until the telescope and receiver behaviors could be quantified and computerized.

Nearly two months into the survey, Jocelyn Bell noticed that there was a repeating tick or 'scruff' on the paper that did not behave like other known sources, seen in Fig. 1.1. Looking back at a previous recording, she realized it had occurred in the same part of the sky (right ascension 19 hours and 19 minutes) before. By measuring

the spacing of the ticks, Jocelyn Bell found that they were occurring every $1 \frac{1}{3}$ seconds 


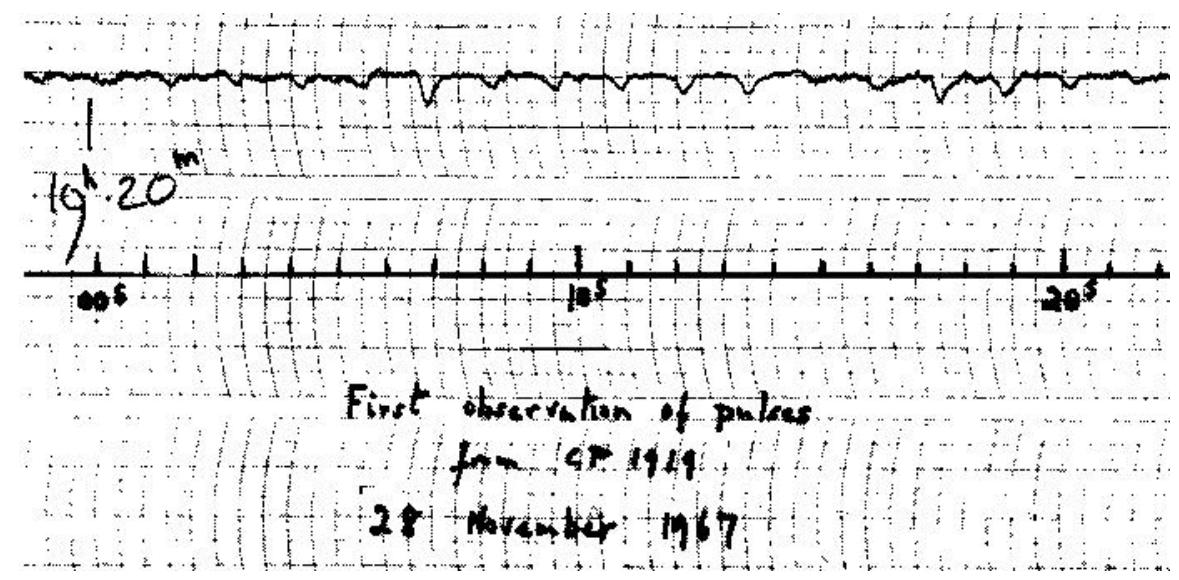

Figure 1.1: The original pen recording of the first discovered pulsar 'CP1919', later renamed PSR B1919+21. The top line is the recorded signal with a downward 'scruff' occurring periodically. Below is the time scale with one second intervals (Hewish et al., 1968).

(Bell Burnell, 1977). Pulses at a simple fraction first appeared manmade, but when Antony Hewish and Jocelyn Bell checked more recordings, they found these pulsations only occurred when that portion of sky was overhead. Whatever this object was, it definitely could not be from Earth.

When the pulsations were observed with another telescope, it eliminated the possibility that it was instrumental in origin (Hewish et al., 1968; Bell Burnell, 1977). Dispersion measurements of the signal determined that the source was outside the solar system and was within the Milky Way.

Such an artificial-looking signal from space had the research team thinking of extraterrestrials and intelligent life. In fact the object was nicknamed LGM-1 for 'little green men'. Antony Hewish then started taking accurate measurements of the pulse period to investigate whether the source originated from an exoplanet orbiting a star. This motion would cause Doppler shifts in the pulse intervals. These measurements only revealed our own orbit around the sun. 
We did not really believe that we had picked up signals from another civilization, but obviously the idea had crossed our minds and we had no proof that it was an entirely natural radio emission. It is an interesting problem - if one thinks one may have detected life elsewhere in the universe how does one announce the results responsibly? Who does one tell first?Bell Burnell (1977)

As more of these sources were discovered and confirmed, it became less likely that many different civilizations scattered throughout the Milky Way would be sending similar pulsed signals toward Earth. Because of this, a more natural explanation was needed. In the discovery paper (Hewish et al., 1968), it was agreed that these sources had to be a type of star. Therefore, it should come as no surprise that these 'pulsating stars' were quickly abridged to become known as 'pulsars'.

\subsection{Pulse origins}

When pulsars were found in supernova remnants, it was quickly pointed out that the signals originated from a class of star known as a neutron star (Staelin \& Reifenstein, 1968; Large et al., 1968). Neutron stars begin their lives as normal mainsequence stars. These stars are in equilibrium, where there is a balance between the compressing gravity forces and the expanding internal pressure forces. For most of their existence, these main-sequence stars are able to maintain this balance effectively. Eventually, the star runs out of 'fuel' for nuclear fusion to generate the necessary 
radiation pressure to hold off gravity. If massive enough, the star will explode as a supernova.

During the supernova explosion, the core of the star collapses, which can result in two different extreme objects. In the most massive stars, gravity will compress all the material to form a black hole, which is an object so dense that light itself cannot even escape the effects of its gravitational potential. If the mass is insufficient to form a black hole, a neutron star is formed by the compression of electrons and protons into neutrons and neutrinos.

Because of this compression, neutron stars are highly compact, smaller than some major cities, and massive, with masses up to nearly two times the mass of the Sun (Oppenheimer \& Volkoff, 1939; Bombaci, 1996). As the star collapses, the magnetic flux and angular momentum are conserved; this causes the magnetic field strength on the surface of the star to reach typical levels of $10^{12}$ Gauss and rotating nearly once every second (Lorimer \& Kramer, 2005).

Within a year of the discovery of the first pulsar, Gold (1968) proposed a scenario where a spinning neutron star has an off-axis dipole magnetic field. This scenario creates a 'lighthouse' effect where the magnetic field confines the

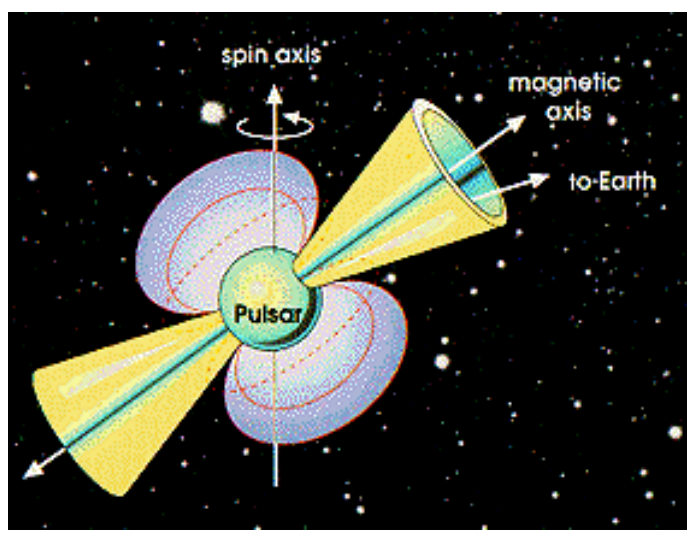

Figure 1.2: A simple diagram from Mattson et al. (2010) of the pulsar model, where the spin axis and the magnetic axis are offset. This combined with a confined emission cone produces a pulsed signal towards Earth.

emission to a narrow beam that only points toward Earth once with each rotation, 
as seen in Fig. 1.2. This situation best explains why the pulses occur so rapidly and regularly.

One of the first attempts to mathematically model this situation was by Goldreich \& Julian (1969), who considered a model with a neutron star which had an aligned rotation and magnetic axes, seen in Fig. 1.3. Along with the fact that these aligned axes will not generate a pulsed signal, there are several reasons why this model is not entirely realistic (see criticisms by Michel \& Li, 1999). Yet, it demonstrates the basic principles that are useful in understanding observed behaviors of pulsars (Lorimer \& Kramer, 2005). Because of this, we quickly review the Goldreich-Julian model to lay the ground work for future discussions in this thesis.

In the Goldreich-Julian model the star itself is believed to be a conductor, implying that electromagnetic forces cancel within the material. A charge $q$ in this material will be co-rotating with an angular velocity of $\Omega$, which gives the particle a linear velocity

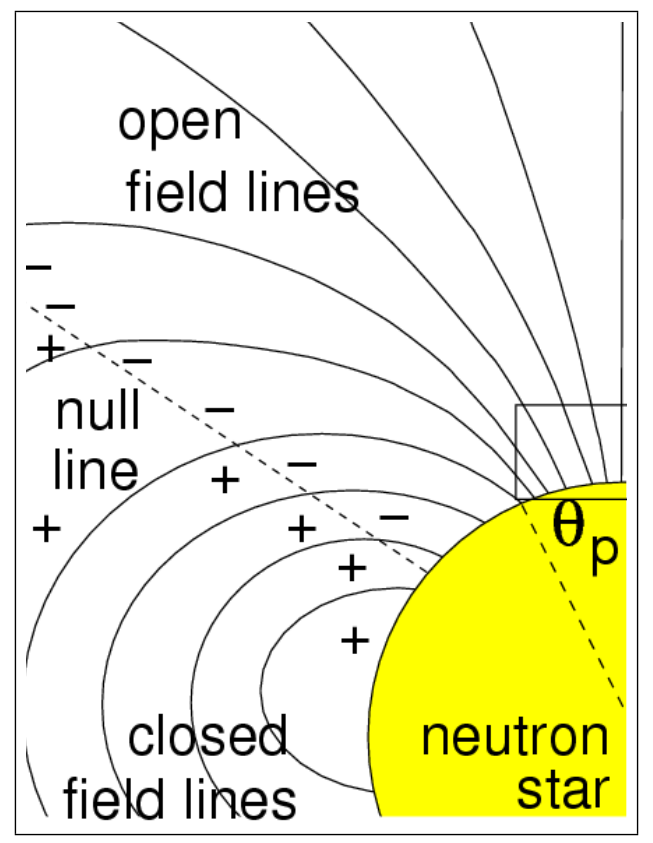

Figure 1.3: The Goldreich-Julian model

$$
\mathbf{V}=\Omega \times \mathbf{r},
$$

as presented in Lorimer \& Kramer (2005) where the magnetic and the rotational axes are aligned vertically. 
where $\mathbf{r}$ is the location vector of the particle. This velocity results in a force

$$
\mathbf{F}=q(\boldsymbol{\Omega} \times \mathbf{r}) \times \mathbf{B}
$$

from the magnetic field, B. Within the conducting material of the star this magnetic force is cancelled by an electric field $\mathbf{E}$ such that

$$
\mathbf{F}=q \mathbf{E}+\frac{q}{c}(\boldsymbol{\Omega} \times \mathbf{r}) \times \mathbf{B}=0
$$

In order to generate such an internal electric field, there must be a surface charge on the star. Assuming the exterior is in a vacuum, this surface charge would produce a quadrupole potential $\Phi_{\text {vac }}$ around the star

$$
\Phi_{\mathrm{vac}}(r, \theta)=\frac{B_{S} \Omega R^{5}}{6 c r^{3}}\left(3 \cos ^{2} \theta-1\right)
$$

where $(r, \theta)$ are polar coordinates originating from the center of the star, $B_{S}$ is the magnetic field strength on the surface, $R$ is the radius of the star and $c$ is the speed of light. This potential generates an electric field at the surface such that

$$
E_{\mathrm{vac}_{\|}}=\left.\frac{\mathbf{E}_{\mathrm{vac}} \cdot \mathbf{B}}{B}\right|_{r=R}=-\frac{\Omega B_{S} R}{c} \cos ^{3} \theta
$$

This force $\left(F=q E_{\mathrm{vac}_{\|}}\right)$on a charged particle located on the surface exceeds the gravitational force by 10 orders of magnitude or more for a typical pulsar. This quickly accelerates the particles into the surroundings to form a dense plasma environment. 
This dense plasma acts as an extension of the conducting material such that the forces cancel, as in Equation 1.3. The way the charges are distributed throughout the environment results in a charge density

$$
\rho_{e}(r, \theta)=\frac{\nabla \cdot \mathbf{E}}{4 \pi}=-\frac{\Omega \cdot \mathbf{B}}{2 \pi c}=-\frac{B_{S} \Omega R^{3}}{4 \pi c r^{3}}\left(3 \cos ^{2} \theta-1\right) .
$$

From this equation, we can see that there is an expected change in the polarity about $\cos \theta=\sqrt{1 / 3}$, shown in Fig. 1.3. If it is assumed that there is a complete charge separation, the charge density has its maximum at the magnetic pole, (i.e. where $r=R$ and $\theta=0)$. This is the Goldreich-Julian density

$$
\rho_{\mathrm{GJ}}=\frac{\Omega B_{S}}{2 \pi c} \simeq \frac{B_{S}}{c P}
$$

where $P$ is the pulse period.

The emission from pulsars is a product of these charges interacting with the magnetic field. High-energy radiation results from charged particles being accelerated along curved magnetic field lines which generates radiation similar to synchrotron known as curvature radiation (Condon \& Ransom, 2010). High-energy photons produced by this curvature radiation interact with the magnetic field and lower-energy photons to produce electron-positron pairs that radiate more high-energy photons (Goldreich \& Julian, 1969). This cascade process eventually produces bunches of charged particles that emit coherently at radio wavelengths larger than the size of the bunch (Condon \& Ransom, 2010). These coherent volumes are larger at lower 
radio frequencies $f$, making them the dominant feature (Condon \& Ransom, 2010). This effect in combination with the curvature radiation gives most pulsars extremely steep radio spectra. Spectral intensities, $S$, follow a power law $\left(S \sim f^{-\alpha}\right)$ where the exponent $\alpha$ is the spectral index of the pulsar (Condon \& Ransom, 2010). Typical (negative) pulsar spectral indices are $\alpha=1.7$, although some can be much steeper $(\alpha=3)$ and a handful are almost flat $(\alpha=0.5)$ (Condon \& Ransom, 2010).

While there seems to be a firm understanding of the basic emission mechanisms, observed emission variations often challenge this knowledge. Therefore, being able to reveal and classify these diverse changes to the emission can give us further insight into their underlying mechanism. Because of this, we will introduce several known emission variation classifications in Section 1.3. Surprisingly, some emission variations are accompanied by changes in the spin dynamics. As a result, we will introduce the basic pulsar spin dynamics and some of these recent discoveries in Section 1.4. One such study (Lyne et al., 2010) has shown that the spin dynamics are coupled with changes in the pulse profile and have features that may be chaotic in nature. Consequently, in Section 1.5 we review classic chaotic behaviors that may be present in these spin-down rate changes.

\subsection{Observed emission variations}

As mentioned in the previous section, if all pulsars had constant emission, where every pulse is identical, the emission mechanism could be conceivably understood. However, in reality, the emission varies substantially from pulse to pulse in very sur- 
prising ways. In the subsections below, we review a few examples of these variations that makes the pulsar emission mechanism so complex. For in-depth discussions on pulsar emission variations see Lorimer \& Kramer (2005) or Lyne \& Graham-Smith (2012).

\subsubsection{Drifting sub-pulses}

When Drake \& Craft (1968) examined individual pulses from the first known pulsar PSR B1919+21 and other newly discovered pulsars, they noticed that prominent features would slowly move across the pulse shape or profile from pulse to pulse. An example is shown in Fig. 1.4a. The drifting sub-pulse features from PSR B1919+21 and PSR B2016+28 were later shown to have an even spacing and occurred periodically. Backer (1973) systematically defined all these different timescales as $P_{1}$ for the time between each main pulse, $P_{2}$ as the time between each sub-pulse, and $P_{3}$ as the time for the sub-pulse pattern to repeat.

Because these sub-pulse patterns occurs so regularly, often a Fourier transform along a fixed longitude or phase bin will return a peak at this pattern's frequency. The frequencies, in this Fourier spectrum referred as the fluctuation spectrum, are often expressed in terms of cycles per pulse period $\left(\mathrm{C} / P_{1}\right)$ (Lorimer \& Kramer, 2005).

The widely accepted explanation of this phenomenon is the carousel model shown in Fig. 1.4b, where there are several sub-beams circulating in a hollow cone centered on the magnetic axis (Ruderman \& Sutherland, 1975). We see a certain cross-section of this cone depending on our line of sight. Under these assumptions, 

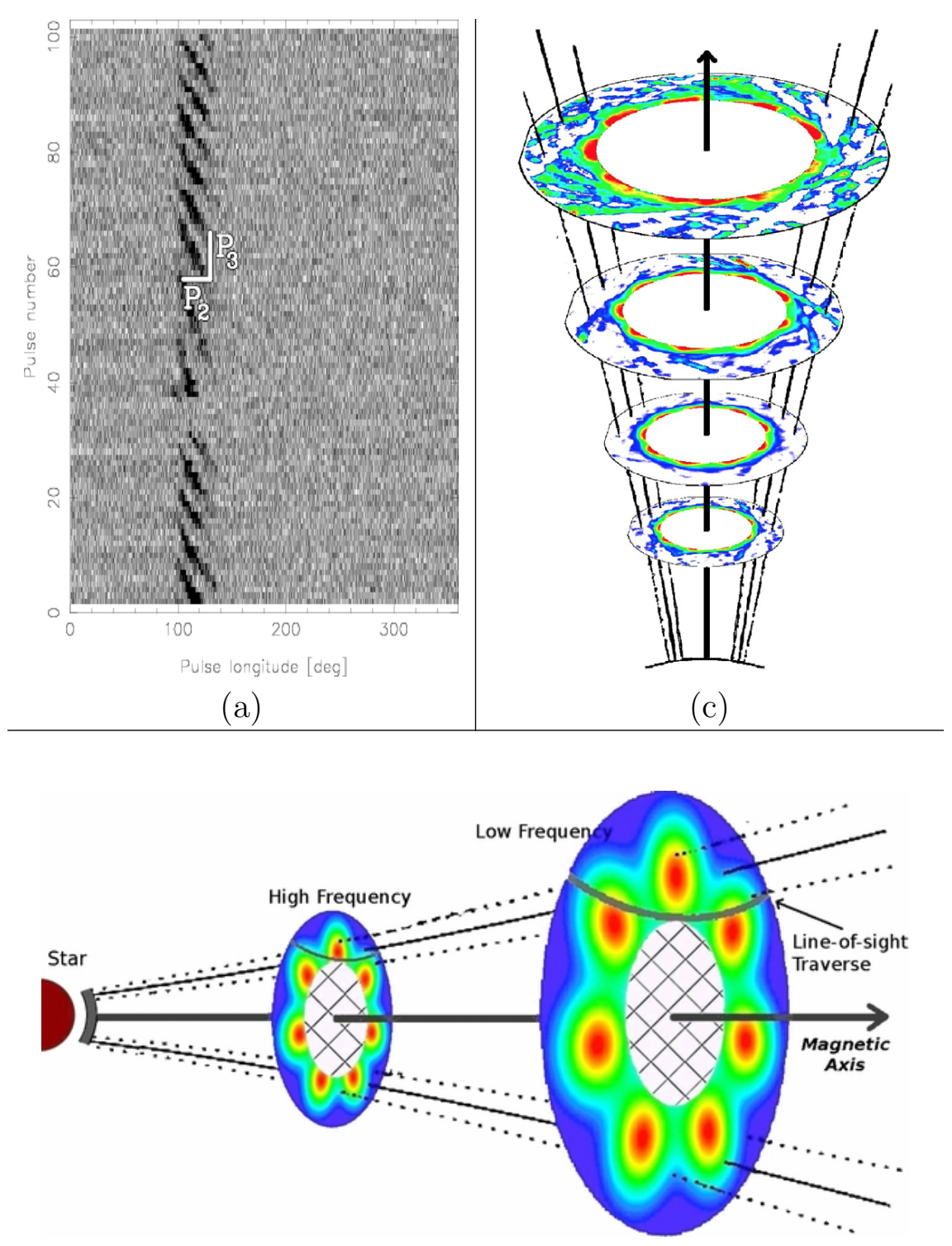

(b)

Figure 1.4: Drifting sub-pulses: (a) An observation with drifting sub-pulses from Serylak et al. (2009) for PSR B0031-07 observed at $92 \mathrm{~cm}$ with the Westerbork Synthesis Radio Telescope. Here the horizontal axis is the pulse longitude and the vertical axis is the pulse number during this observation. Along with this, $P_{1}$ is the pulse period and is the duration of the horizontal axis, $P_{2}$ is the time between subpulse, and $P_{3}$ is the time for the pattern to repeat. (b) An example of the carousel model presented in Maan et al. (2013). The size of the inaccessible inner region (cross-hatched) is determined by how close the line of sight gets to the magnetic axis. (c) An example of a cartographic transform of B0809+74 (for details, see Deshpande \& Rankin (1999)) presented by Maan et al. (2013) for four $16 \mathrm{MHz}$ frequency bands (centered at 117, 172, 233, and $330 \mathrm{MHz}$ ) observed with the Robert C. Byrd Green Bank Telescope. 
it is possible to recreate this sub-pulse geometry with a technique known as the cartographic transform (Deshpande \& Rankin, 1999). Based on $P_{2}$ and $P_{3}$, the cartographic transform converts the received intensities onto a rotating frame centered on the magnetic axis of the pulsar, an example of which can be seen in Fig. 1.4(c) (Deshpande \& Rankin, 1999). This is done by converting the pulse longitudes to different radial distances away from the magnetic axis such that the loop closes after $P_{3}$. The effectiveness of this mapping has repeatedly supported the model in Ruderman $\&$ Sutherland (1975).

\subsubsection{Nulling}

While observing additional moving features in pulse profiles was confusing, seeing a pulsar miss a beat or null is even more perplexing. More precisely, nulling is when the pulses abruptly disappear for several to hundreds of pulses and the emission suddenly returns as if it never left (Backer, 1970d). This can be seen in Fig. 1.4a around 30 pulses into the observation. At the extreme end of the nulling phenomenon are the rotating radio transients (RRATs). These objects sporadically emit pulses that occur at integer multiples (McLaughlin et al., 2006).

An effective analysis for determining nulling is looking at the energy statistics of the pulses (Smith, 1973). These energies are calculated by integrating the flux density across each pulse. By examining the distributions of these pulse energies, it can easily be determined whether the nulling that is being seen originates with the pulsar or is caused by missed pulses due to the telescope's sensitivity. If the nulling events 
were a simple result of the telescope's sensitivity, the energy values would produce a smooth distribution that includes zero. Yet if the nulling events are separate events, the energy values produce a combination of two distributions with one distribution centered on zero. An example of this is shown in the right side of Fig. 1.5, where a clear bimodal distribution can be seen. While this shows that nulling events are distinct, we will see that the results in Chapter 3 will call into question whether some nulling events truly have no flux or whether they are instead extremely weak pulses.

Although not universal, an analysis of pulse nulling across the population of isolated pulsars by Wang et al. (2007) tends to suggest that the fraction of the time spent in the null state increases with characteristic age (see Section 1.4.1). Because pulsar emission is thought to originate in the magnetosphere, nulling is believed to be a direct result of changes within this region.

This, coupled with the age correlation, has caused some authors to think that nulling is caused by a diminishing supply of charged particles from the surface
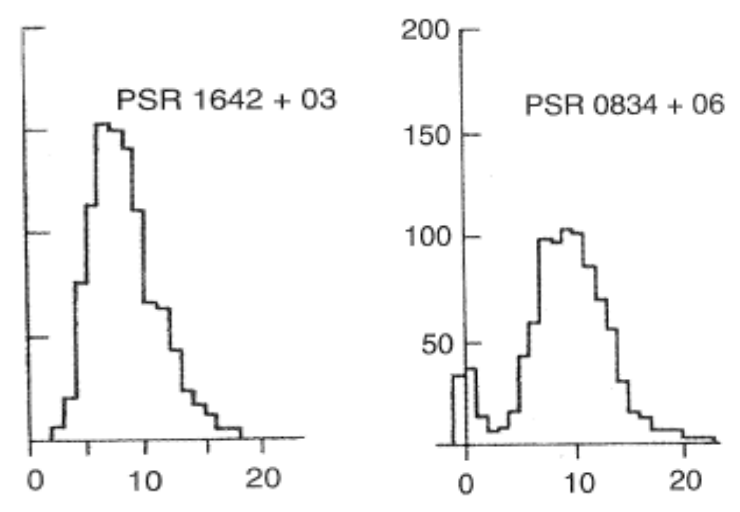

Pulse Energy (arbitrary units)

Figure 1.5: Distributions of single pulse energies for two pulsars from Smith (1973) observed at $408 \mathrm{MHz}$ with the Lovell radio telescope. On the left is the energy distribution for PSR $1642+03$, which has no nulling events that results in a smooth continuous distribution with only one peak. The right hand side is the energy distribution for PSR $0834+06$, which has nulling events. The nulling events form a second peak centered around zero.

(Manchester \& Taylor, 1977; Ruderman \& Sutherland, 1975; Sturrock, 1971). Shannon et al. (2009) has explored the possibility that asteroid interactions with the mag- 
netosphere may cause nulling. However, because of the greatly varying time scales on which nulling occurs a general explanation has so far not been found.

\subsubsection{Mode switching}

The dual personality of pulsars does not stop with nulling. In fact nulling may be related to another phenomenon known as mode switching (Wang et al., 2007). Mode switching is where the pulse profiles abruptly switch between two or more recurring stable pulse shapes (Backer, 1970c). Mode switching pulsars usually favor a particular mode where it spends most of the time. It has become common practice to label this favored mode as the normal mode.

As with nulling, mode switching will usually show a bimodal distribution in the pulse energies, but both modes will peak at non-zero energy values. The pulses of each mode can be isolated and averaged separately to form a mean profile for each state. The average mode profiles of J1326-6700 are shown in Fig. 1.6, where we can see drastic changes in the pulse profile between each emission mode.

Because mode switching and nulling both happen abruptly, occur on similar timescales, form bimodal energy distributions, and imply changes within the magnetosphere, it is widely believed that nulling is a particular emission state of the mode switching phenomenon (Wang et al., 2007). 


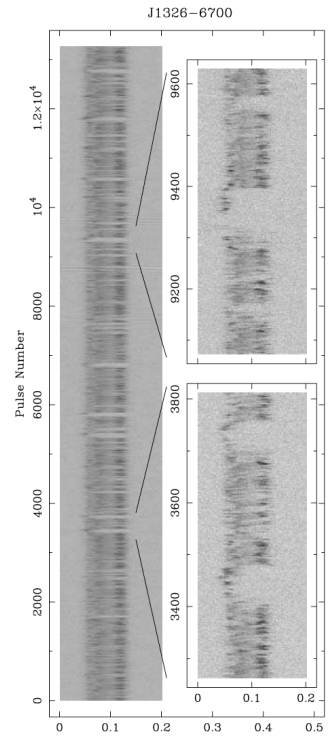

(a)

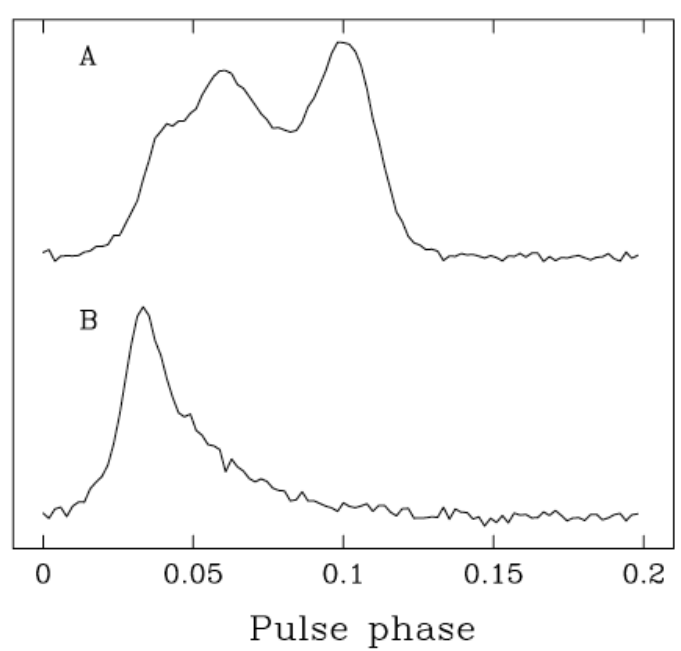

(b)

Figure 1.6: Mode switching in J1326-6700 observed at $1518 \mathrm{MHz}$ with the Parkes radio telescope that was originally presented in Wang et al. (2007). (a) Pulse number versus phase. Here we can see that there are clear recurring changes over several consecutive pulses. (b) The mean pulse profiles of the two modes.

\subsubsection{Bursting}

Both mode switching and nulling appear to be steady, with little change when in a particular state. Yet over the past decade it has become apparent that a new class of pulsars exist with the behaviors discovered in PSR J1752+2359 (Lewandowski et al., 2004) and PSR J1938+2213 (Chandler, 2003; Lorimer et al., 2013), where a sharp increase in the emission systematically drops off from pulse to pulse, as seen in Fig. 1.7a. These 'bursting pulsars' bear some resemblance to those which exhibit nulling/mode switching. After the bursts, as shown in Fig. 1.7b, the pulsar abruptly returns to a null or faint state.

Bursting may be a lot more common than just the above two pulsars. As seen in Chapter 2, we have been able to reveal bursting events in PSR B0611+22, a pulsar 


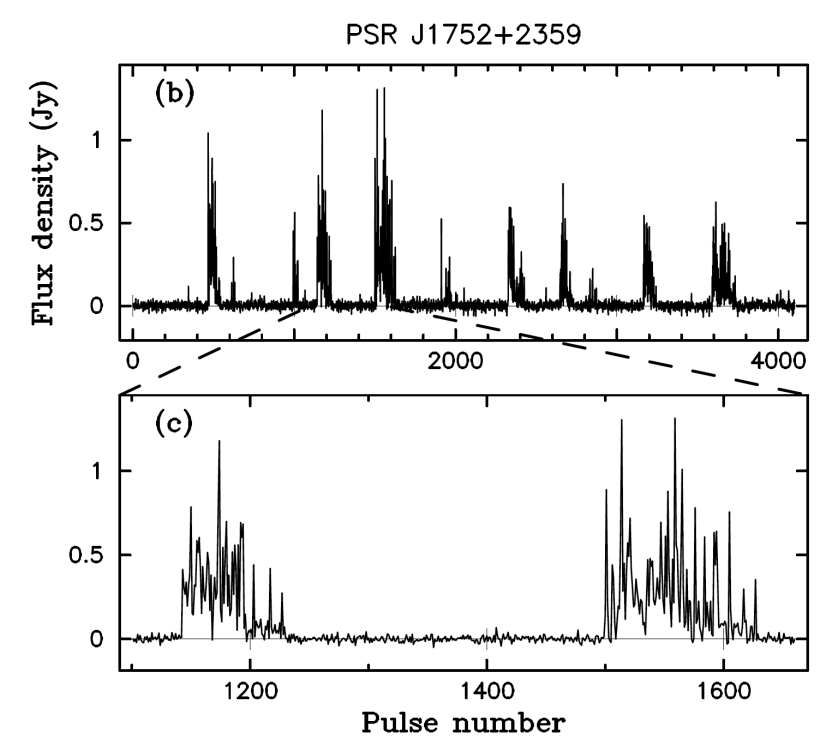

(a)

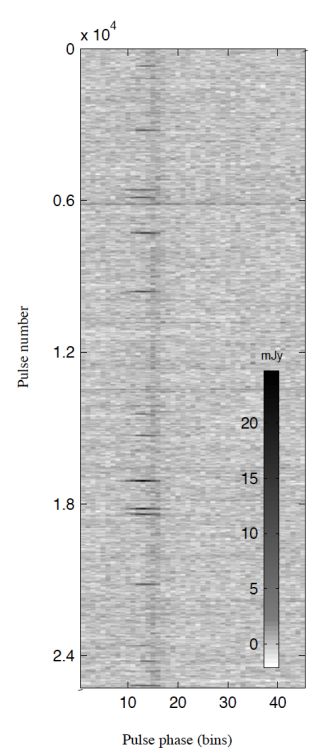

(b)

Figure 1.7: Bursting phenomena: (a) Flux versus pulse number for PSR J1752+2359 at $430 \mathrm{MHz}$ with the Arecibo observatory as presented in Lewandowski et al. (2004). Here we can see the decaying structure of the bursting events into what is considered to be a null state. (b) Pulse number versus pulse phase in bins for PSR J1938+2213 from Lorimer et al. (2013) which was observed at $20 \mathrm{~cm}$ with the Parkes radio telescope. Here the bursting events are the isolated black regions and the companion mode is a faint vertical stripe.

that has been observed for 40 yrs. Also shown in Chapter 2, the bursting events in PSR B0611+22 are similar to mode switching with apparent changes in the mean profile between the bursting and non-bursting events.

\subsection{Emission variations accompanied by spin-down fluctuations.}

In recent years, unexpected discoveries have shown that changes in the emission can be accompanied by changes in the spin dynamics. To understand why these changes are so unexpected, why they may be occurring, and to introduce some of the terminology, we first need to look at the expected spin dynamics of a pulsar. 


\subsubsection{Basic pulsar spin dynamics}

When pulsars are measured over time, their pulse periods increase. This increase in period implies that a star's rotation is slowing down. This slowdown requires a braking torque. By tracking the energy losses, we can see where this torque originates.

First, the rate at which the rotational energy, $E$, is being lost will be

$$
\dot{E}=-\frac{d E}{d t}=-\frac{d\left(I \omega^{2} / 2\right)}{d t}=-I \omega \dot{\omega}=4 \pi^{2} I \dot{P} P^{-3}
$$

where $I$ is the moment of inertia of the star, $\omega=\frac{2 \pi}{P}$ is the angular velocity, $P$ is the pulse period, and the dot above a quantity indicates a time derivative. If this energy loss is equal to the radiation power that is expected for a spinning magnetic dipole with a moment $|m|$, then we have

$$
\dot{E}=\frac{2}{3 c^{3}}|m|^{2} \omega^{4} \sin ^{2} \alpha,
$$

where $\alpha$ is the angle between the rotational and magnetic axis and $c$ is the speed of light (Jackson, 1962). Setting these equal we find that

$$
\dot{\omega}=-\left(\frac{2|m|^{2} \sin ^{2} \alpha}{3 I c^{3}}\right) \omega^{3} .
$$

This dynamical equation can then be converted to observable quantities,

$$
\dot{\nu}=-K \nu^{n},
$$


where $K$ assumed to be constant, $\nu=\omega /(2 \pi)$ and is the pulse frequency and the braking index, $n$, takes the value 3 in the rotating magnetic dipole model. Because $\dot{\nu}$ is negative, the spin frequency decrease with time. Thus, $\dot{\nu}$ is often referred to as the slowdown or spin-down rate of the pulsar.

From the above equation, it is possible to estimate the pulsar's age by knowing $\nu=1 / P$, solving for time, and assuming the initial period was much smaller than it is now. This age estimate, $\tau=P / 2 \dot{P}$, is known as the characteristic age.

Because of this relationship, $P$ and $\dot{P}$ are plotted against one another to trace the evolution of different pulsars, seen in Fig. 1.8. Under the previous assumptions, pulsars will follow lines of constant magnetic field strength as they age. Often binary pulsars can be spun-up by accretion of material from their companion star (Bhattacharya \& van den Heuvel, 1991), which reduces their period and causes them to migrate from the main group of pulsars in Fig. 1.8.

Admittedly, the pulsar environment is not simply dominated by magnetic dipole radiation. Therefore, $n$ varies from pulsar to pulsar. Some factors that can affect the braking index are listed in Table 1.1 (for an in depth discussion see Manchester \& Taylor, 1977). These braking indices are obtained for different characteristics of the pulsar. By changing the electromagnetic properties of the star, models have been able to incorporate multipole electromagnetic radiation, magnetic field decay, and plasma effects that deform the magnetic field lines. Varying the angle between the spin and the magnetic axis over time produces an additional effect on the braking that may either enhance the braking index, when these axes become more aligned, or reduce it, when the axes become counteraligned or drift apart (Manchester \& Taylor, 


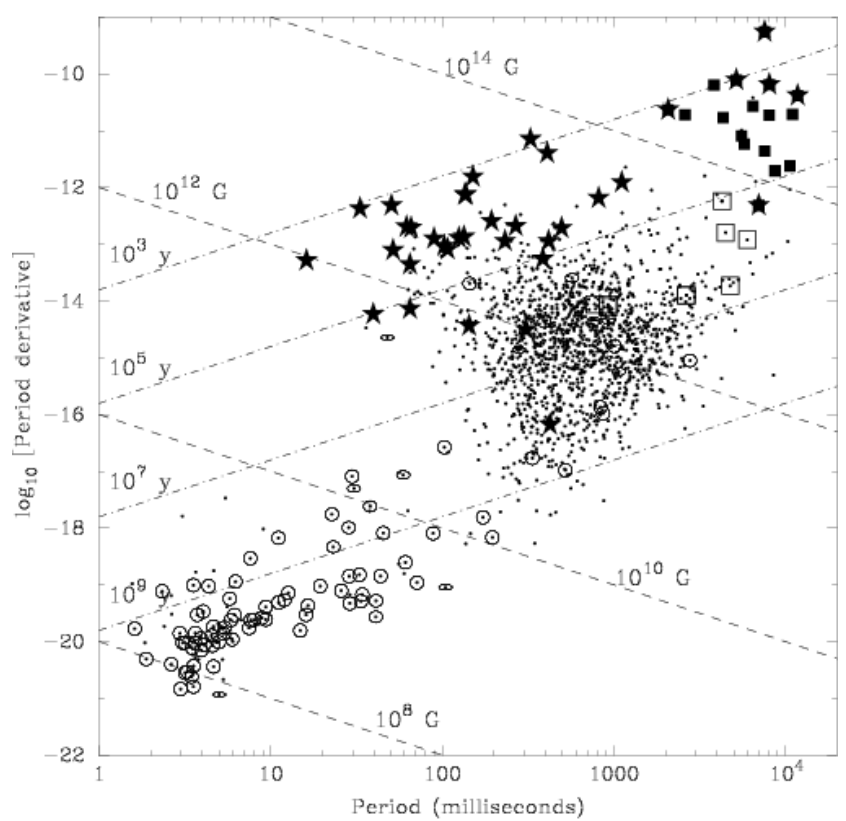

Figure 1.8: $P-\dot{P}$ diagram from Lorimer (2011) showing normal pulsars as dots, binary pulsars as circles or ellipses, magnetars as filled squares, RRATs as open squares and supernova remnant associations as filled stars. Lines of constant characteristic age $\tau$ and magnetic field strength are also shown. As a pulsar ages, it is theorized that it will follow lines of constant magnetic field strength downwards and to the right.

1977). Assuming a non-spherical star, it is theorized that the braking index will be equal to 5 because of the energy loss of gravitational radiation, but this has yet to be observed (Ostriker \& Gunn, 1969; Manchester \& Taylor, 1977). Others have investigated the possibility that the moment of inertia changes as the pulsar slows down, causing a relaxation of its equilibrium (Manchester \& Taylor, 1977). Also, there can be a perceived effect on the braking index which is not intrinsic to the pulsar that is a result of the observed transverse motion (Shklovskii, 1969; Manchester \& Taylor, 1977).

Regardless, pulsars are massive objects that have been compressed to nearly perfect spherical proportions. Their dynamics are incredibly stable, with the fastest spinning and oldest 'millisecond pulsars' generally being the most predictable. Petit 


\begin{tabular}{lc} 
Situations which affect the braking index & Braking index \\
\hline Multipole electromagnetic radiation & $n \geq 5$ \\
Gravitational quadrupole radiation & $n=5$ \\
Alignment of dipole field & $n>3$ \\
Decay of magnetic field & $n>3$ \\
Radial deformation of field-lines & $1 \leq n \leq 3$ \\
Counter-alignment of dipole field & $n<3$ \\
Relaxation of equilibrium-form of neutron star & $n<3$ \\
Transverse velocity & $n<3$
\end{tabular}

Table 1.1: List of situations and their theoretical effects on the braking index from Manchester \& Taylor (1977)

\& Tavella (1996) have shown that these millisecond pulsars can be as stable as atomic clocks on large timescales.

\subsubsection{Abrupt spin-down rate variations}

Fluctuations in the spin-down rate of pulsars was highly unexpected due to their stability and are a rather new discovery. These changes are only seen by combining several observations, because of the timescale on which they occur. Yet, the most abrupt changes are the best understood and have a similar explanation.

\subsubsection{Intermittent pulsars}

Intermittent pulsars have prolonged nulling and continuous emission events, each occurring on timescales of weeks to months. When inspecting the rotational frequency of the pulses over time, it becomes apparent that the emitted pulses do not follow the long term spin-down rate, as seen in Fig. 1.9A. Rather, it appears that the emitting events have a $50 \%$ steeper decline than the long term value, implying a larger 
and therefore a different spin-down rate when nulling occurs. In fact by abruptly switching between two different spin-down rates for each emission state, the pulse arrival times can be precisely modeled. As seen in Fig. 1.9B, the timing residuals, which are the differences between the observed pulse arrival times and those derived from the simple long-term slowdown model, indicate a different frequency derivative for each state (Kramer et al., 2006).

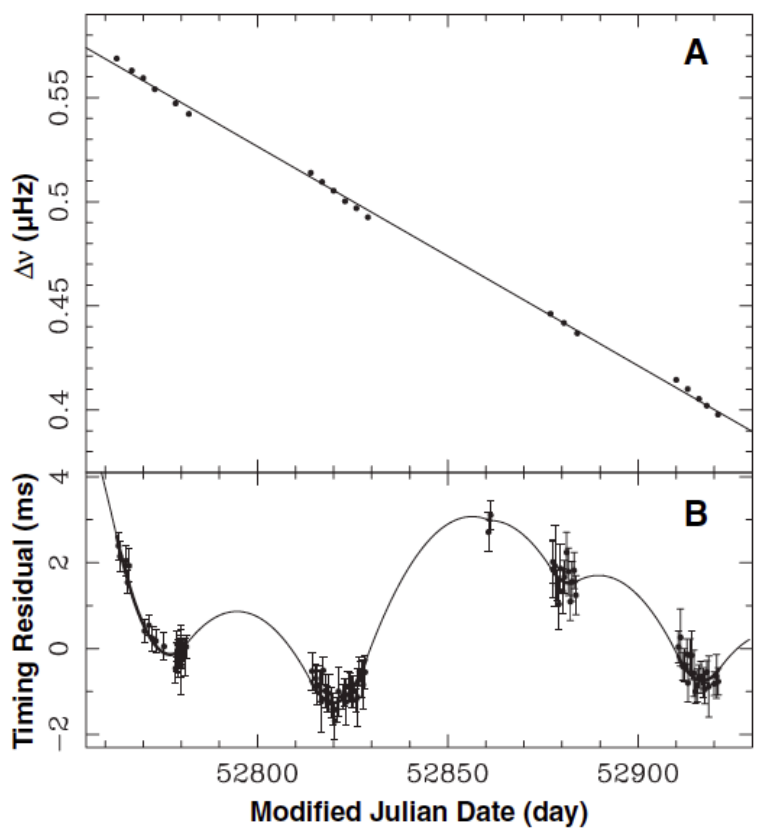

Figure 1.9: Spin frequency and timing residuals of the intermittent pulsar B1931+24 presented in Kramer et al. (2006). (A) Evolution of the rotational frequency over a 160 day period. The spin dynamics are dominated by a long-term spin-down indicated as a best-fit solid straight line through the points. However, when the pulsar is on, the slope and hence the spin-down rate increases. This suggests different spin-down rates during the off and on states. (B) The timing residuals that fit well with different frequency derivatives for each state (solid curve).

These large changes in spin-down rate that accompany the appearance of radio emission can be explained by fluctuations in the plasma charge density around the pulsar. This plasma, thought to be the source of coherent radio emission, would 
create a current flow that can account for an additional braking torque on the pulsar

$$
T \sim \frac{2 \pi}{3} \rho B_{S} R_{\mathrm{pc}}^{3}
$$

where $B_{S}$ is the dipole magnetic field at the neutron star surface, and $R_{p c}$ is the radius of the polar cap region with charge density $\rho$ (Kramer et al., 2006). The charge density can be calculated by tracking the loss of rotational energy, via Equation 1.8, during the on, $\dot{E}_{r_{\text {on }}}$, and off, $\dot{E}_{r_{\text {off }}}$, phases. The additional effect of the plasma density implies an energy loss for the on phase of

$$
\dot{E}_{r_{\mathrm{on}}}=\dot{E}_{r_{\mathrm{off}}}+\dot{E}_{T}
$$

where

$$
\dot{E_{T}}=2 \pi T \nu=\frac{4 \pi^{2}}{3} \rho B_{S} R_{\mathrm{pc}}^{3}
$$

Inserting the above $\dot{E}_{T}$ equation and Equation 1.8 for each state into Equation 1.13 results in a plasma density of

$$
\rho=\frac{3 I\left(\dot{\nu}_{\mathrm{off}}-\dot{\nu}_{\mathrm{on}}\right)}{R_{\mathrm{pc}}^{4} B_{S}},
$$

where $I$ is the moment of inertia and $\dot{\nu}$ is the spin-down rate during the phase of the subscript. When Kramer et al. (2006) did this calculation for B1931+24, they found that the charge density required for such a torque is $\rho=0.034 \mathrm{C} \mathrm{m}^{-3}$, which is remarkably similar to the Goldreich-Julian density, $\rho_{\mathrm{GJ}}=0.033 \mathrm{C} \mathrm{m}^{-3}$ for this star. 
Because these values are so similar, changes in charge density can reasonably explain the different spin-down rates for each state, but it is not clear what determines the long timescales or what could be responsible for suddenly changing the plasma flow in the magnetosphere (Kramer et al., 2006). Understanding the radiation disruptions in B1931+24 may also help us understand the origins of ordinary nulling (Kramer et al., 2006).

\subsubsection{Component appearance and drifting}

Another relationship between emission and spin-down has been seen recently from J0738-4042 in Brook et al. (2014), where there has been an appearance of a slow drifting component that increases in flux density until reaching a particular spin phase after several years (see Fig. 1.10). The appearance of this component is accompanied by a sudden increase, followed by an oscillation, in the spin-down rate before converging around a new steady rate, as seen in Fig. 1.10G.

These changes are believed to be from an asteroid interaction, where a small body, falling toward a pulsar, is evaporated and ionized by the pulsar radiation (Brook et al., 2014). This charged material can be electrically captured by the pulsar which increases the emission and reduces the net charge density surrounding the pulsar (Cordes \& Shannon, 2008; Brook et al., 2014), which is the opposite of what was seen in the previous section. Some charged particles are accelerated to relativistic energies, producing gamma rays, which give rise to a cascade of electron-positron pairproduction (Cordes \& Shannon, 2008). This pair-production can enhance or diminish 

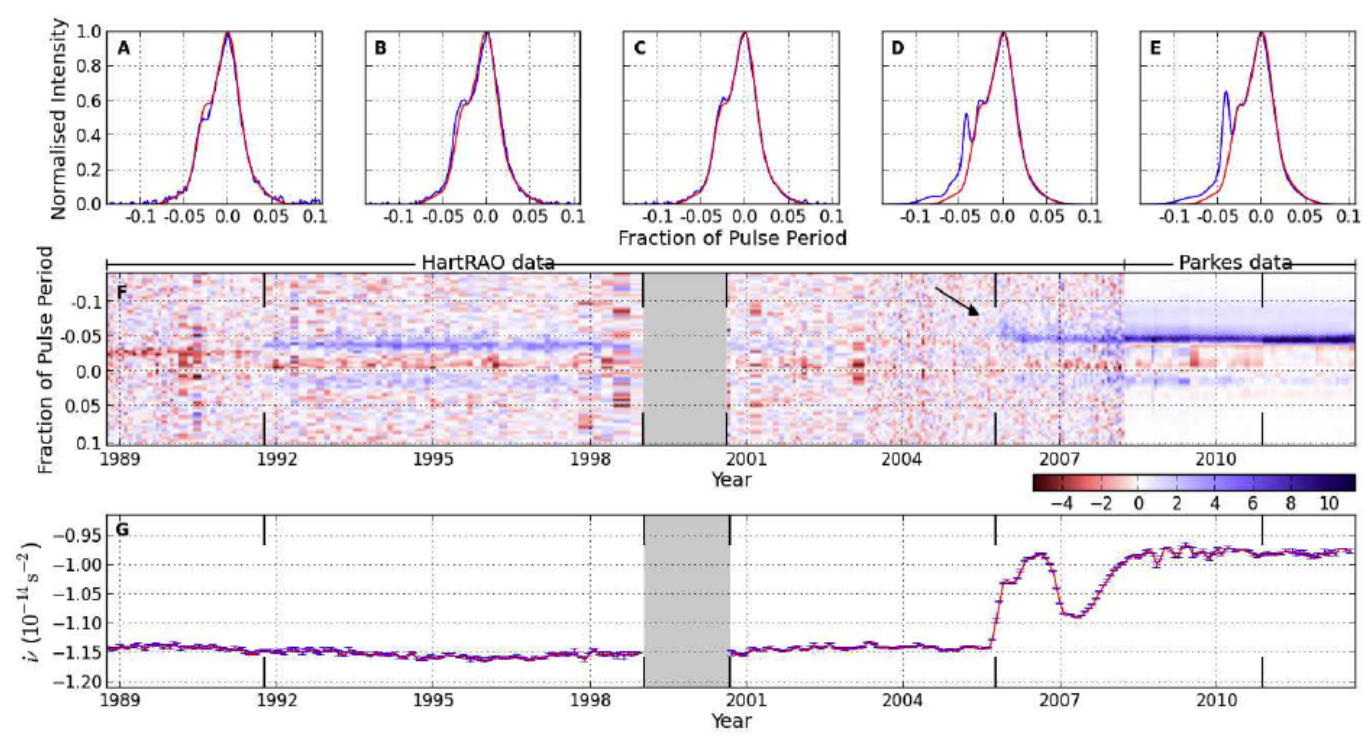

Figure 1.10: Variations in the profile shape and spin-down rate seen in J0738-4042 presented in Brook et al. (2014). Profiles were observed at $1600 \mathrm{MHz}$ with HartRAO and at $1369 \mathrm{MHz}$ with the Parkes telescope. Panels A-E: The blue trace denotes the median pulse profile for each of 5 intervals over the 24-year dataset, which are demarcated in panels $\mathrm{F}$ and $\mathrm{G}$. The red trace in each plot is a constant model profile which represents the median profile for all of the HartRAO data. Panel F: Map showing the difference between data and the constant model, in units of the HartRAO off-pulse standard deviation. The epochs at which data were collected from both telescopes were used to normalize the Parkes data to the HartRAO scale. The arrow points to drifting emission changes which precede the emergence of a new persistent profile component. Panel G: Pulsar spin-down rate as a function of time.

the emission within certain regions (Cordes \& Shannon, 2008; Brook et al., 2014), which can be seen as a component appearing or disappearing in the pulse profile. Because of the magnetic geometry of the pulsar, Cordes \& Shannon (2008) also states that such a change could also affect the emission height for a given frequency, Brook et al. (2014) attribute the phase shift of the component to a decrease in the emission height.

The reduction of the net charge density also results in a reduction in the braking torque (Brook et al., 2014). When Brook et al. (2014) inserted the values for J0738-4042 into Equation 1.15, they found a charge density drop of $\rho=0.007 \mathrm{C} \mathrm{m}^{-3}$. 
Converting this charge density to a mass density, Brook et al. (2014) approximated a mass flow rate by assuming relativistic speeds, $v \sim c$, across the polar regions. Given its current duration, the mass amounts to $\sim 10^{15} \mathrm{~g}$, which lies within the known range of solar system asteroids and is consistent with the mass range of asteroids around neutron stars proposed by Cordes \& Shannon (2008) (Brook et al., 2014). For this scenario, it is predicted that the pulsar will return to its previous spin-down rate after the injected mass has been fully depleted (Brook et al., 2014).

\subsubsection{Continuous spin-down rate variations}

Since long-term dynamical fluctuations in pulsars were unexpected, the bulk of the dynamical irregularities were considered to be 'timing noise', random errors about a kinematic model (Hobbs et al., 2006). When Lyne et al. (2010) observed some of these irregularities for more than 20 yrs, large timescale fluctuations in the spin-down rate became clear, as seen in Fig. 1.11a. Lyne et al. (2010) describe these irregularities as 'quasi-periodic' and notice that changes in spin-down rate were often accompanied by changes in the pulse duration or width. When the pulse width measurements were compared with the spin-down fluctuations, a clear correlation exists, as seen in Fig. $1.11 b$.

At least two different processes have been proposed to explain these fluctuations. Jones (2012) argues that free precession is one of the few processes that can occur on such long timescales. This paper also addresses the comments made in Lyne et al. (2010) that free precession is not likely because sudden fluctuations in the spin- 


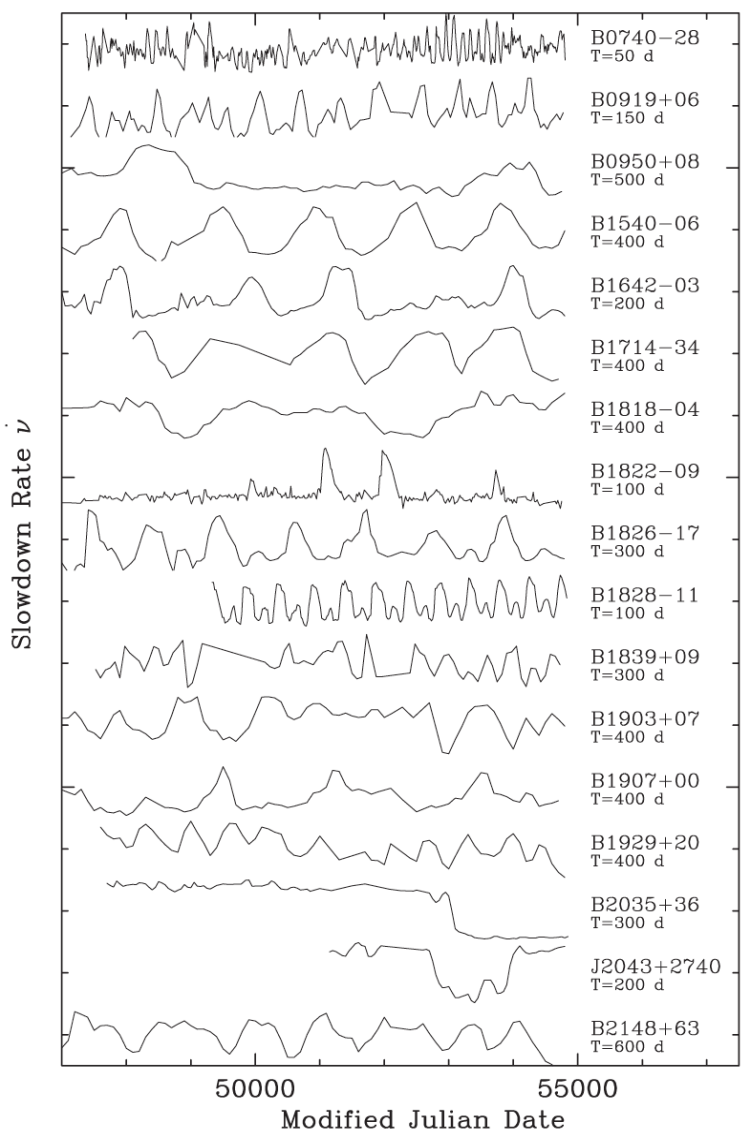

(a)

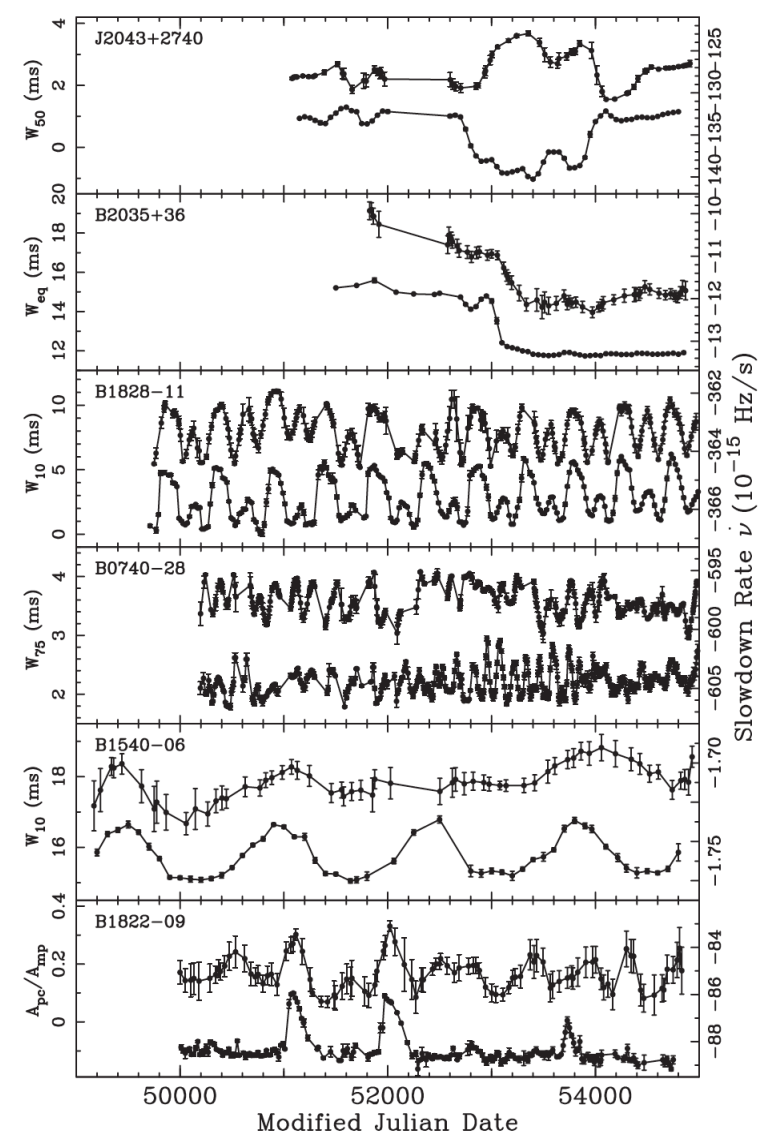

(b)

Figure 1.11: Spin-down rate variations presented in Lyne et al. (2010) (a) Slowdown rate changes for 17 pulsars. (b) Pulse width and spin-down changes for 6 pulsars. In each panel, there is a measurement of the pulse width (top) and the spin-down (bottom) changes. The two quantities are strongly correlated or anti-correlated.

down rate are accompanied by profile changes, seeming to suggest disturbances in the magnetosphere. Jones (2012) also proposed a test for this model where future observations need to find pulsars with very different periods than the ones listed in Lyne et al. (2010). Admittedly, Jones (2012) states that the fluctuations in larger period pulsar would be on too long of timescale to be seen, and, as seen in Fig. 1.8, finding shorter period $(P<100 \mathrm{~ms})$ isolated pulsars with this behavior is also unlikely. 
Another model presented in Rosen et al. (2011) suggests that velocity variations in the surface of the star influence the magnetosphere and the spin-down. The variations are due to the presence of non-radial oscillation modes at the stellar surface. These modes have rather short periods with the long-term variations being caused by an excitation of a single mode, or the redistribution of energy over several modes. The Rosen et al. (2011) model can only account for purely periodic fluctuations and Jones (2012) debates that this non-radial oscillation model does not provide an explanation for the long-term periodic structures seen in Lyne et al. (2010).

The preceding discussion shows that the debate continues concerning a mechanism that governs these long term spin-down rate fluctuations and their connection to the pulse shape. For all these fluctuations to have a similar cause, there must be a rather complex underlying interaction. In light of these uncertainties, it would be helpful to develop additional diagnostics to understand the level of complexity for these quasi-periodic behaviors.

\subsection{Chaotic behaviors}

One possible relationship for the changes seen in Lyne et al. (2010) is that these fluctuations are part of a non-linear chaotic system. Often the term quasi-periodic is also used to describe some of the behaviors seen in these systems. In everyday conversation, the word chaos is often interchangeable with randomness, but in dynamical studies these are two distinct ideas. Chaos is continuous and deterministic, 
while randomness is more complex and uncorrelated; values at an earlier time have no effect on the values at a later time.

One of the characteristics of chaos has been colorfully described by Edward Lorenz (1993) as the 'butterfly effect'. Lorenz asks, 'Does the flap of a butterfly's wings in Brazil set off a tornado in Texas?' This is used to illustrate an instability, where a system is highly sensitive to initial conditions. The consequence is that if there is a small displacement in the initial conditions, the difference between the two scenarios will grow exponentially to cause significant changes at a later time. This instability does not arise in linear dynamics and is a chaotic phenomenon in non-linear systems (Scargle, 1992). We will utilize this chaotic trait in Section 4.2.2.

An example of this behavior is shown in Fig. 1.12 for the Lorenz system of equations

$$
\begin{aligned}
& \dot{x}=\sigma(y-x) \\
& \dot{y}=x(\rho-z)-y \\
& \dot{z}=x y-\beta z .
\end{aligned}
$$

Here $\sigma, \rho$, and $\beta$ are positive parameters. Lorenz (1963) derived this system from a simplified model of convection rolls in the atmosphere. In the original derivation $\sigma$ is the Prandtl number, $\rho$ the Rayleigh number and $\beta$ has no proper name but relates to the height of the fluid layer. As for the governing variables, $x$ is proportional to the intensity of the convective motion, $y$ is proportional to the temperature difference of the ascending and descending currents, and $z$ is proportional to the distortion of the vertical temperature profile from linearity (Lorenz, 1963). Since then, the Lorenz equations have appeared in a wide range of physical systems. 


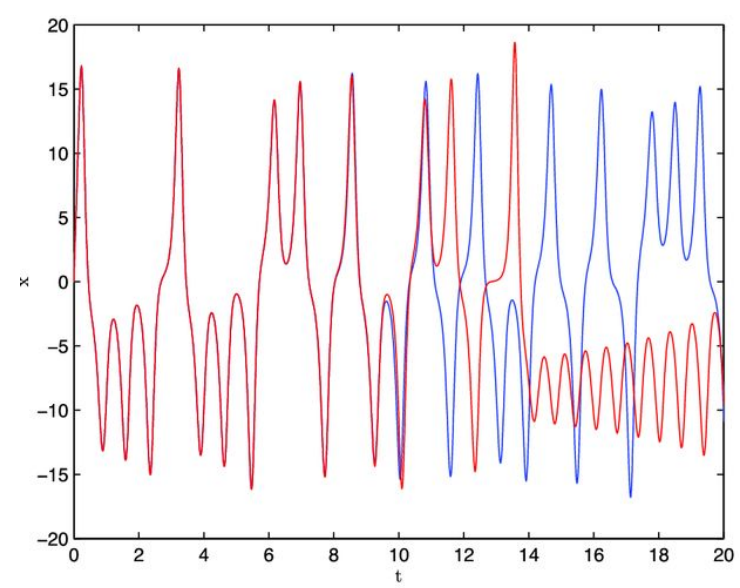

(a)

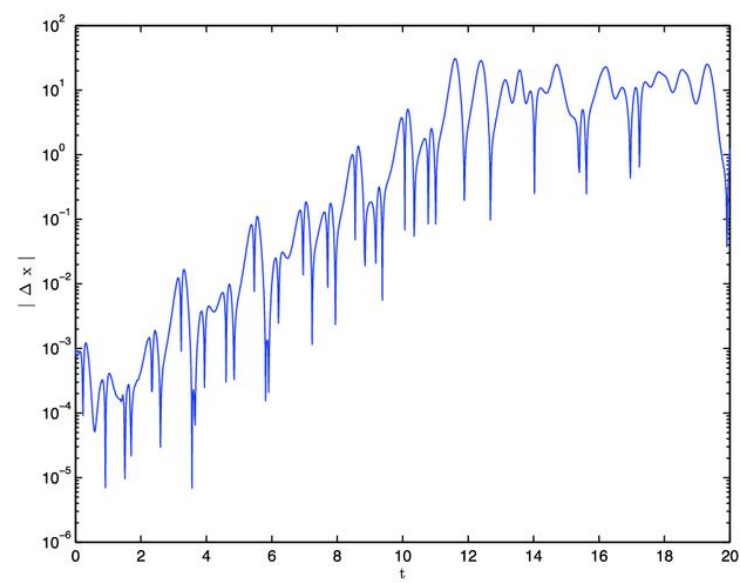

(b)

Figure 1.12: (a) Two $x$ component solutions for the Lorenz equations over time whose initial conditions differed by $10^{-3}$ in $x$. (b) The difference between the two scenarios in (a) over time. An overall increasing exponential trend can be seen.

It is important to note that there are no analytical solutions to most nonlinear equations. Often to produce a solution, as used in Fig. 1.13, the system is numerically solved with small enough time steps where linear relationships can be used to approximate a function. We can see from the governing equations that any function that is produced will be highly dependent on the other variables. When these time functions are plotted with respect to each other, as seen in Fig. 1.13a, they trace out a rather odd surface.

The Lorenz equations are not the only system in which this occurs. Rössler (1976) was in search of a simpler set of equations with similar chaotic behavior to the Lorenz attractor. He came up with with the following three equations with only one non-linear term $z x$

$$
\begin{aligned}
& \dot{x}=-y-x \\
& \dot{y}=x+a y \\
& \dot{z}=b-z(x-c) .
\end{aligned}
$$




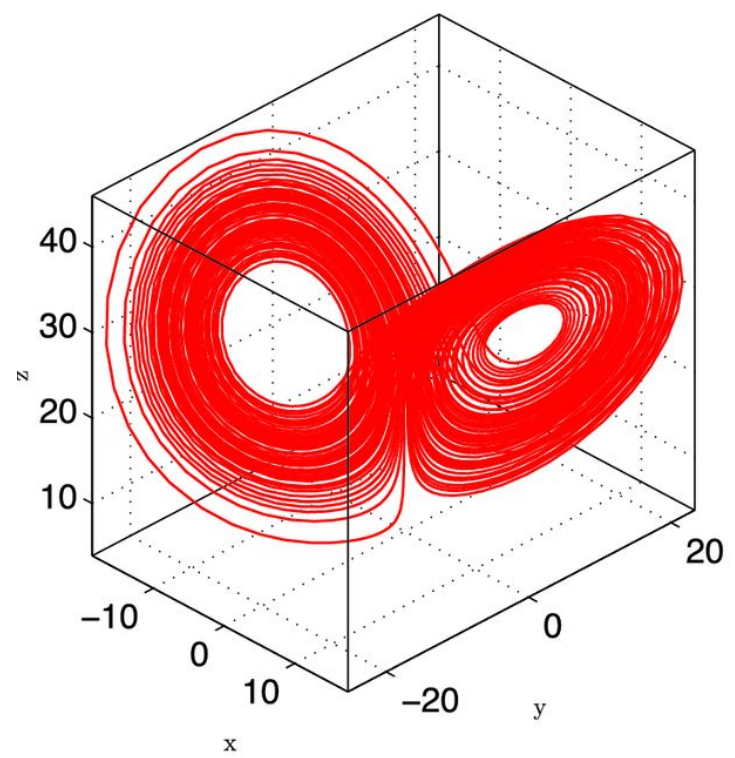

(a)

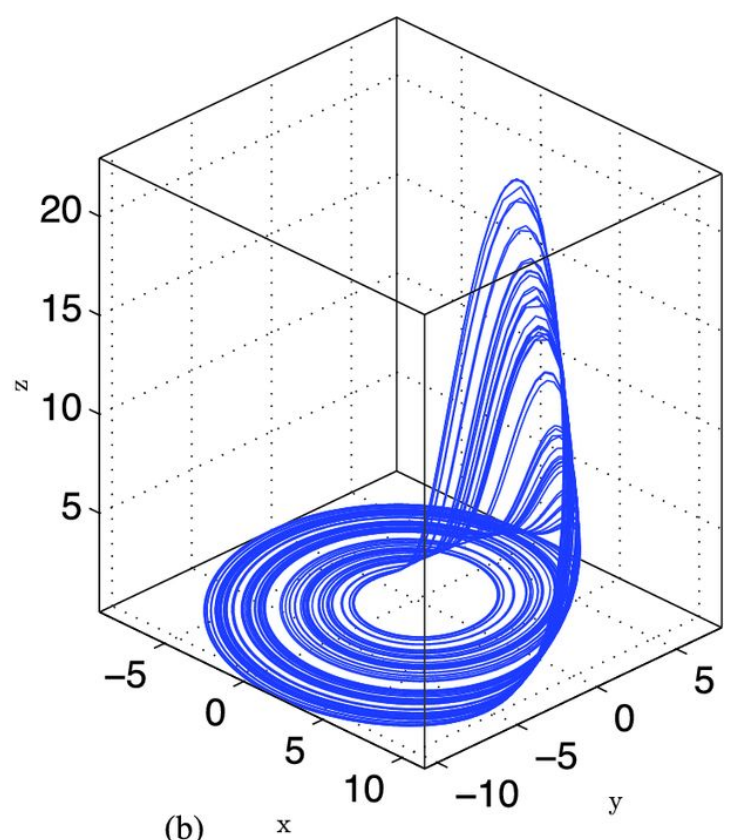

(b)

Figure 1.13: Three dimensional visualization of two chaotic attractors. ( $a$ ) The Lorenz attractor from $t=0$ to 100 with $\sigma=10, \rho=28, \beta=8 / 3$ and $x_{0}=0, y_{0}=10$, $z_{0}=10.2$. (b) The Rössler attractor from $t=0$ to 500 with $a=0.2, b=0.2, c=5.7$ and $x_{0}=-1.887, y_{0}=-3.5, z_{0}=0.09789$

Here $a, b$, and $c$ are parameters. Again, plotting the variables against each other produces a different bizarre surface, seen in Fig. 1.13b. Though the Rössler equations started solely as a mathematical construct, analogous behavior has been seen in chemical reactions (Argoul et al., 1987).

The complex shapes that the dynamics form are known as 'strange attractors'. They are called 'attractors' because, regardless of the initial conditions, the functions will converge to a path along these surfaces. They are 'strange' because they are fractal in nature. The dimension of the attractor will be a non-integer that is less than the number of equations. We will discuss this more in Section 1.5.1.

The convergent path along the attractor is controlled by the parameters in the governing equations. When a control parameter is slowly increased, the system 

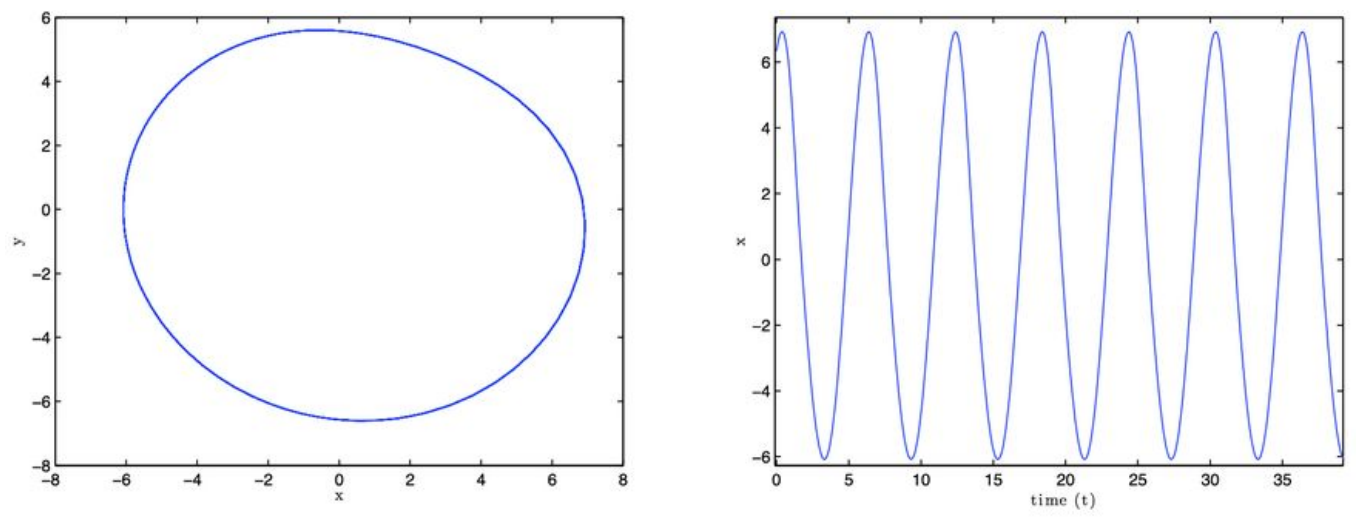

Periodic $c=4$
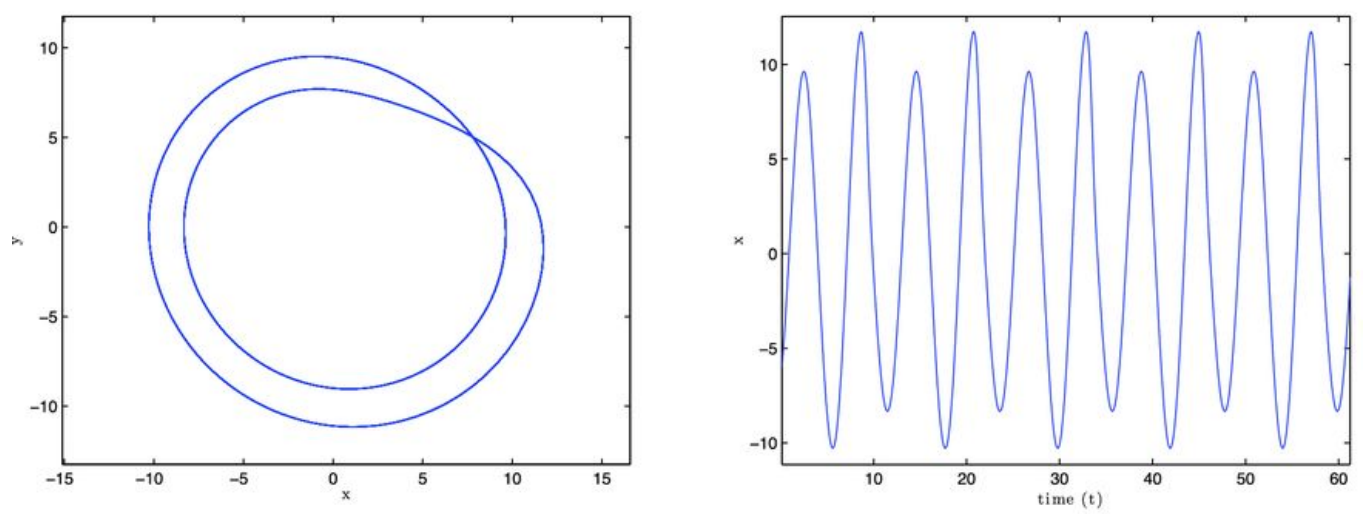

\section{Period two $c=7$}

-

-

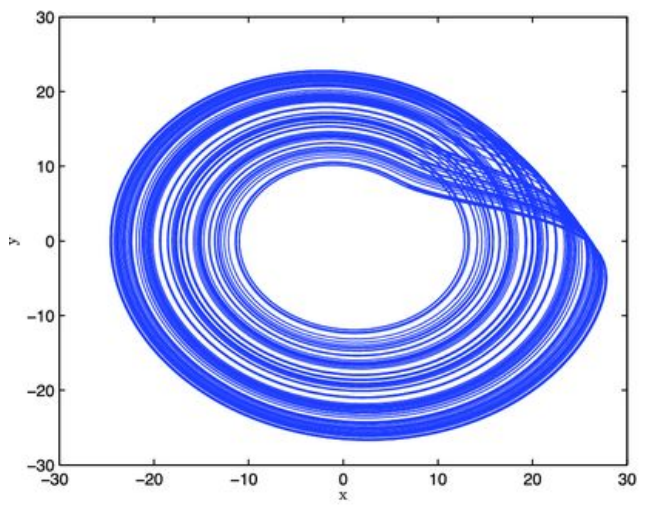

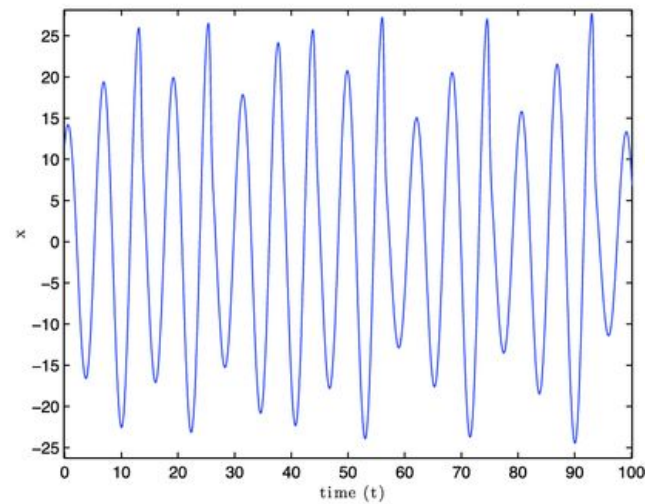

Chaotic $c=18$

Figure 1.14: The route to chaos for the Rössler equations with $a=b=0.1$. Left side is the $\mathrm{x}-\mathrm{y}$ projection for each case. On the right is the $\mathrm{x}$ component verses time. 
exhibits a series of behaviors (Scargle, 1992). This series is known as the 'route to chaos'. The behaviors usually unfold as: constant $\Rightarrow$ periodic $\Rightarrow$ period two $\Rightarrow \ldots$ chaos (Olsen \& Degn, 1985), as demonstrated in Fig. 1.14, where 'period two' refers to a repeating path that travels twice around the attractor. As the parameter is increased, this behavior continues to where the path cycles three, four, or more times to return to the same location, until it suddenly becomes chaotic, where the path will never repeat.

\subsubsection{Fractal dimensions}

We often see dimensions as the minimum number of coordinates needed to describe every point in a given geometry (Strogatz, 1994). For example, a smooth curve is one dimensional because we can describe every point by one number, the distance along the curve to a reference point on the curve (Strogatz, 1994). This definition fails us when we try to apply it to fractals, and we can see why by examining the von Koch curve.

Shown in Fig. 1.5.1, the von Koch curve is an infinite limit to an iterative process. We start with a line segment $S_{0}$ and break the segment into three equal parts. The middle section is swung 60 degrees and another section of equal length is added to close the gap to form segment $S_{1}$. This process is repeated to each line segment to produce the next iteration.

With each iteration, the curve length is increased by a factor of $\frac{4}{3}$. Therefore, the total length at an iteration $n$ will be $L_{n}=L_{0} \times\left(\frac{4}{3}\right)^{n}$. When this is iterated an 
infinite amount of times, it produces a curve with an infinite length. Not only that, there would be an infinite distance between any two points on the curve.

We would not be able to describe every point by an arc length, but we would be able to describe them with two values, say the Cartesian coordinates. Our original definition would define this as two-dimensional. However, this does

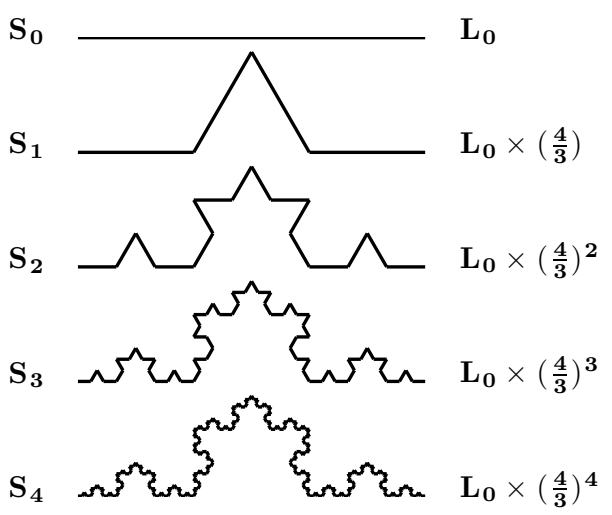
not make sense intuitively, since this is not an area. Therefore this is something in between, one plus some fraction of a dimension. This is known as a fractal

Figure 1.15: Iterations leading to the von Koch curve. $S_{n}$ denotes the segment on iteration $n$ and the far right column is the total length of the corresponding segment. The von Koch curve is when $n \rightarrow \infty$. dimension.

\subsection{Thesis outline}

The work presented in the rest of this thesis will be based around searching for the variabilities and behaviors that we have introduced. It is important to reveal and classify the emission variations of pulsars, because it helps develop our knowledge of the emission mechanism and how it is affected. Also, by successfully quantifying these changes, models may more accurately predict these complicated phenomena. 
As we have seen, some emission variations are not isolated from the spin dynamics. For the long-term continuous fluctuations, this connection is still puzzling. Therefore, any efforts that can narrow this search are highly beneficial.

For these reasons, we introduce methods to reveal the emission variations that are present in PSR B0611+22 and investigate their properties in Chapter 2. Next, in Chapter 3, we explore whether PSR B0611+22's variations can give us insight to a known bursting pulsar J1752+2359's emission. An intriguing possibility from the long-term fluctuations observed in Lyne et al. (2010) is that these spin-down changes are chaotic in nature. To investigate this, in Chapter 4 we search for and quantify chaotic behaviors in the spin-down rate measurements from Lyne et al. (2010). Lastly, in Chapter 5, we will draw ties as to how the bursting emission relates to spin-down changes and explain how future work can aid in these connections. 


\section{Chapter 2}

\section{Pulse to pulse study of B0611+22 ${ }^{1}$}

In this chapter, we present a previously unseen bursting emission phenomenon in B0611+22 which was discovered by Davies et al. (1972). This young pulsar with a characteristic age of 90,000 years has considerably high timing noise (Helfand et al., 1980). This high level of noise could indicate that there is still some underlying phenomena that is not being accounted for in the timing models. At first glance, it seems to be a normal pulsar with a simple pulse shape - essentially Gaussian in appearance that is approximately $80 \%$ linearly polarized (Rankin et al., 1989). Yet it has been shown that the integrated pulse shape varies at different times (Ferguson \& Boriakoff, 1980). Nowakowski (1992) has also shown that there is a correlation with the brighter pulses and their phase alinement, and later claimed that the timing noise was due to mode switching (Nowakowski \& Rivera, 2000). Other efforts were made to find a relationship contributing to this timing noise, such as sub-pulse drifts (Weltevrede et al., 2007), but to no avail.

In this chapter, using archival Arecibo observations, we have been able to reveal that this pulsar exhibits bursting episodes and confirms that it has moding behaviors. How this was performed and the implications are outlined in this chapter in the following way, in Section 2.1 we provide technical information about the observations; in Section 2.2 we detail the data analysis techniques used to expose relationships

\footnotetext{
${ }^{1}$ Much of the work presented in this chapter has been published in Seymour et al. (2014).
} 
from pulse-to-pulse; in Section 2.3 we conduct a fluctuation spectrum analysis on the normal and bursting modes found in Section 2.2; finally, in Section 2.4, we discuss our results and their scientific significance.

\subsection{Observations}

The data presented here were collected between March 2 and 8, 2009 (MJD range 54892-54898) in a dedicated observing run with the Arecibo telescope to search for unusual spectral index behavior in B0611+22. To characterize the flux density spectrum, observations were carried out at $327 \mathrm{MHz}, 1400 \mathrm{MHz}, 4.5 \mathrm{GHz}$, and $8.8 \mathrm{GHz}$ using the Wide Band Arecibo Pulsar Processors (WAPPs; Dowd et al., 2000). Unfortunately, the pulsar was not detected at $8.8 \mathrm{GHz}$ and radio frequency interference (RFI) dominated the $4.5 \mathrm{GHz}$ observations. Further details of the individual observations in which the pulsar was clearly detected are shown in Table 2.1.

Table 2.1: Summary of Arecibo observations of B0611+22.

\begin{tabular}{cccccc}
\hline MJD & $\begin{array}{c}\text { Centre Frequency } \\
(\mathrm{MHz})\end{array}$ & $\begin{array}{c}\text { Integration Time } \\
(\mathrm{s})\end{array}$ & $\begin{array}{c}\text { Bandwidth/Channel } \\
(\mathrm{MHz})\end{array}$ & $\begin{array}{c}\text { Sample time } \\
(\mu \mathrm{s})\end{array}$ & Number of Channels \\
\hline 54892.97 & 1400 & 900 & 0.195 & 256 & 2048 \\
54893.01 & 327 & 900 & 0.012 & 256 & 4096 \\
54894.95 & 327 & 900 & 0.012 & 256 & 4096 \\
54894.97 & 1400 & 900 & 0.781 & 128 & 512 \\
54896.94 & 327 & 900 & 0.012 & 256 & 512 \\
54896.97 & 1400 & 900 & 0.781 & 128 & 512 \\
54898.95 & 1400 & 900 & 0.781 & 128 & 4096 \\
54898.97 & 327 & 900 & 0.012 & 256 & \\
\hline
\end{tabular}




\subsection{Data analysis}

The data from each of the observations listed in Table 2.1 were analyzed in a systematic way, as described below.

\subsubsection{Individual pulse profiles}

The autocorrelation functions recorded by the WAPPs in each polarization channel were summed and Fourier transformed to convert them to the equivalent set of total-power spectral channels using standard data analysis techniques (see, e.g., Section 5.2.2 of Lorimer \& Kramer, 2005) implemented in the filterbank program as part of the SIGPROC ${ }^{2}$ pulsar processing package. The resulting data were then dedispersed at the pulsar's dispersion measure $\left(96.91 \mathrm{~cm}^{-3} \mathrm{pc}\right)$ and folded at the predicted pulse period $(0.33 \mathrm{~s})$ using the ephemeris provided in the ATNF pulsar catalog (Manchester et al., 2005) ${ }^{3}$. The sigProc fold program was used to produce an ASCII pulse profile with 256 rotational phase bins. Then using the standard deviation of the off-pulse bins, we were able to convert to mJy using the radiometer equation (see, e.g., Section 7.3.2 of Lorimer \& Kramer, 2005), with a gain of $10 \mathrm{~K} / \mathrm{Jy}$ and an assumed system temperature of $122 \mathrm{~K}$ for $327 \mathrm{MHz}$ and $30 \mathrm{~K}$ for $1.4 \mathrm{GHz}$. Zoomed-in versions of these profiles are shown in the left panels of Figs. 2.1 and 2.3.

\footnotetext{
${ }^{2}$ http://sigproc.sourceforge.net/

${ }^{3}$ www.atnf.csiro.au/people/pulsar/psrcat/
} 


\subsubsection{Revealing the emission modes}

When looking for a relationship across pulse intensities, there is often a great deal of noise present in the system which may obscure any underlying correlation. A simple way to reveal these relationships is to take a boxcar convolution (a running average) over several data points. This method increases the signal-to-noise ratio by the square root of the total number of data points that were averaged, but one must be aware of the fact that this will also reduce the resolution of the data along the direction of the averaging. We carried out a boxcar convolution of the data with a normalized area kernel along 64 data points in a single bin, to preserve the bin resolution. We chose this kernel size to reduce the noise fluctuations by at least a factor of five, and to have a multiple of two for computing efficiency. This averaging is often known as smoothing, and we will refer to these post-processed data as smoothed data.

When smoothing is performed on the $327 \mathrm{MHz}$ emission from B0611+22, the mode changes suggested in Nowakowski (1992) are readily apparent, as shown in the centre panel of Fig. 2.1. There, two prolonged episodes of enhanced emission can be seen. These enhanced emission episodes can then be isolated when the average of each bin is subtracted from that bin to form the perturbations about the mean. These perturbations are then smoothed to produce the right panel of Fig. 2.1. It appears that the enhanced episodes are additional effects overlying a normal emission mode.

To examine the structure of these episodes, a bin was chosen in 

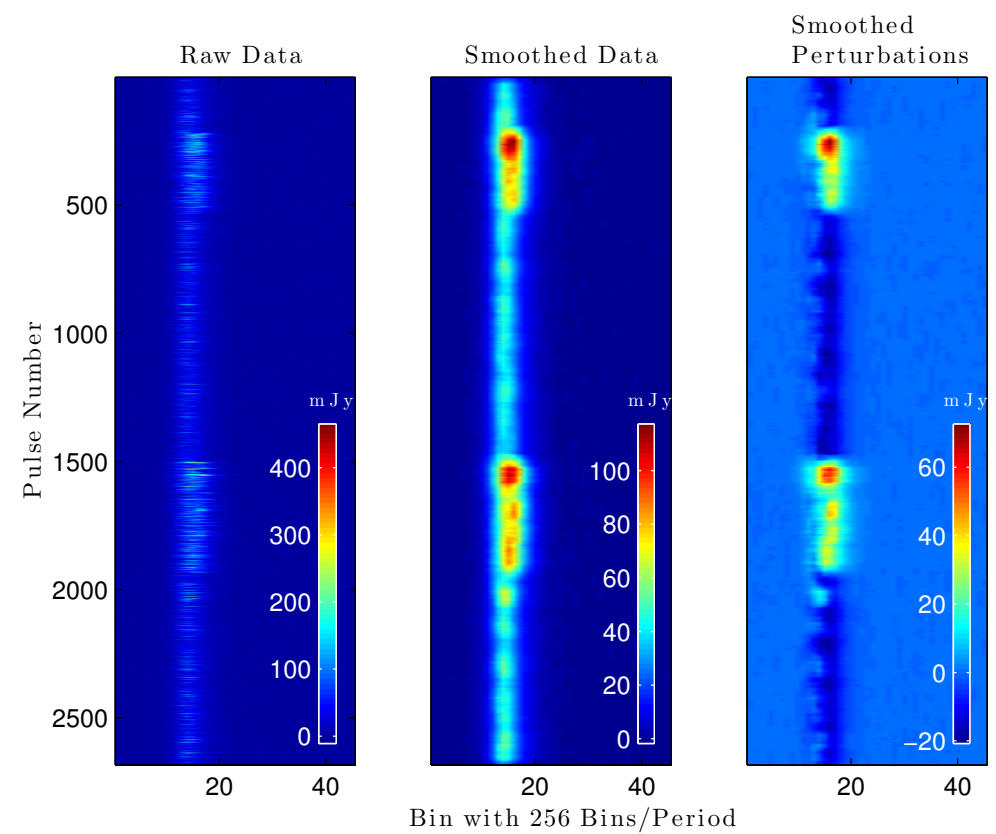

Figure 2.1: The $327 \mathrm{MHz}$ observation of B0611+22 on MJD 54898. Left: The folded dedispersed time series. Center: The time series after being box car convolved along each bin with a kernel of 64 pulses. Right: The convolution of the perturbations about the mean profile with the same kernel.

a section where the abnormalities are the dominant feature. The flux density and its convolution for that bin are shown in Fig. 2.2. There, a decaying behavior can be seen across the event before it abruptly returns to the normal emission mode. This trend has not been recorded for this pulsar before but is strikingly similar to what was reported in Lewandowski et al. (2004) for J1752+2359. There the authors describe

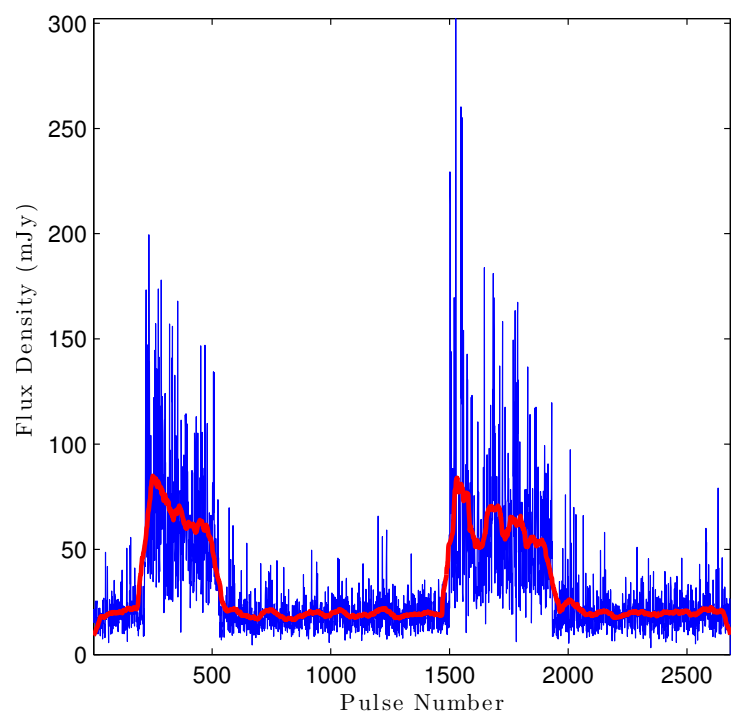

Figure 2.2: Flux along the bin where the bursting events dominate in Fig. 2.1. The solid line is the boxcar convolution of the series. 

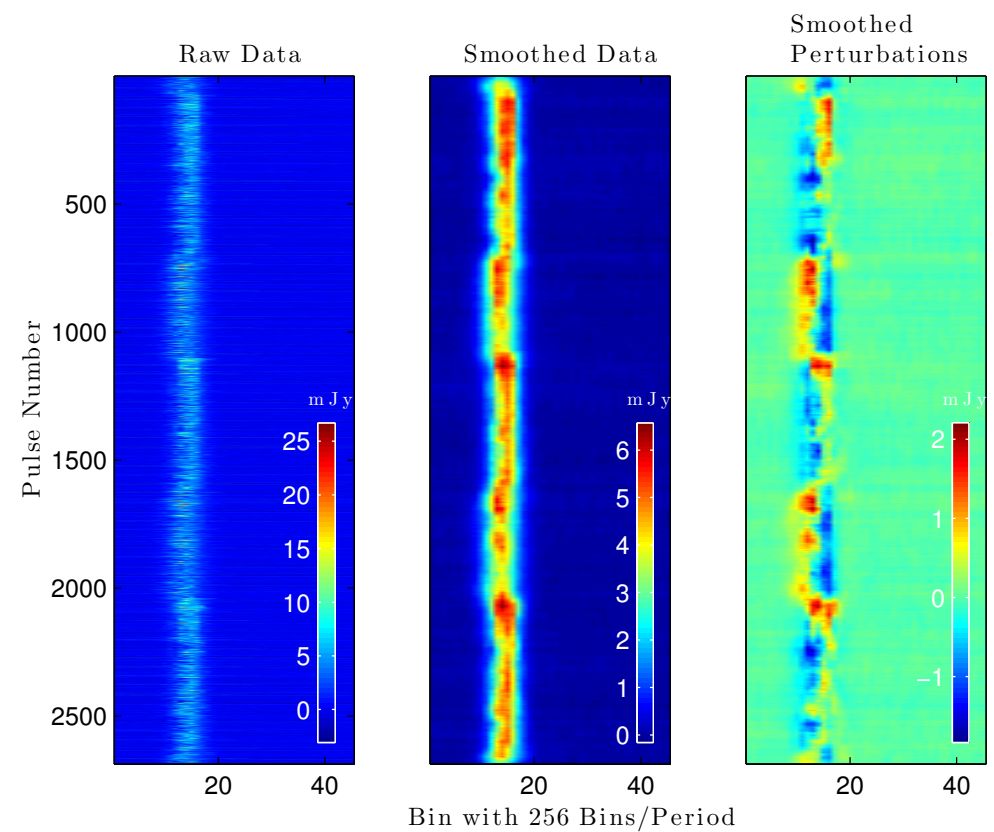

Figure 2.3: The 1.4 GHz observation of B0611+22 on MJD 54898. Left: The folded dedispersed time series with one pulse profile for each pulse. Center: The time series after being box car convolved along each bin with a kernel of 64 pulses. Right: The convolution of the perturbations around the mean profile with the same kernel.

this type of event as bursting. We adopt this nomenclature and will refer to this type of event as a bursting emission mode.

To investigate whether these modes can be seen at other frequencies, the same analysis was conducted on data collected at $1.4 \mathrm{GHz}$. From the perturbations about the mean at this frequency, seen in Fig. 2.3, it becomes clear that there is an undulating relationship. This suggests that mode switching is present.

To examine whether the structures of these modes are similar to the ones at 327 $\mathrm{MHz}$, we plot bin values from either side of the undulation which are shown in Fig. 2.4. On the left side of the undulation, shown in the top of Fig. 2.4, pulse sequences of larger flux density are mesa (i.e. table-top) shaped with a quite constant mean value throughout before quickly returning to a baseline value. On the other hand, the 
right-sided enhanced sequences, shown in the bottom of Fig. 2.4, appear to have the decaying structure of the bursting mode.

This therefore confirms that the two modes found at $327 \mathrm{MHz}$ are also present at $1.4 \mathrm{GHz}$, but with intriguing differences. Most notably, at $1.4 \mathrm{GHz}$ the bursting mode in the right hand bin is no longer accompanied by a steady weaker mode in the left hand bin. In the top of Fig. 2.4 we can see that the left bin is far from steady and at this higher frequency it exhibits abrupt changes of its own. Moreover, the left hand bursts al-
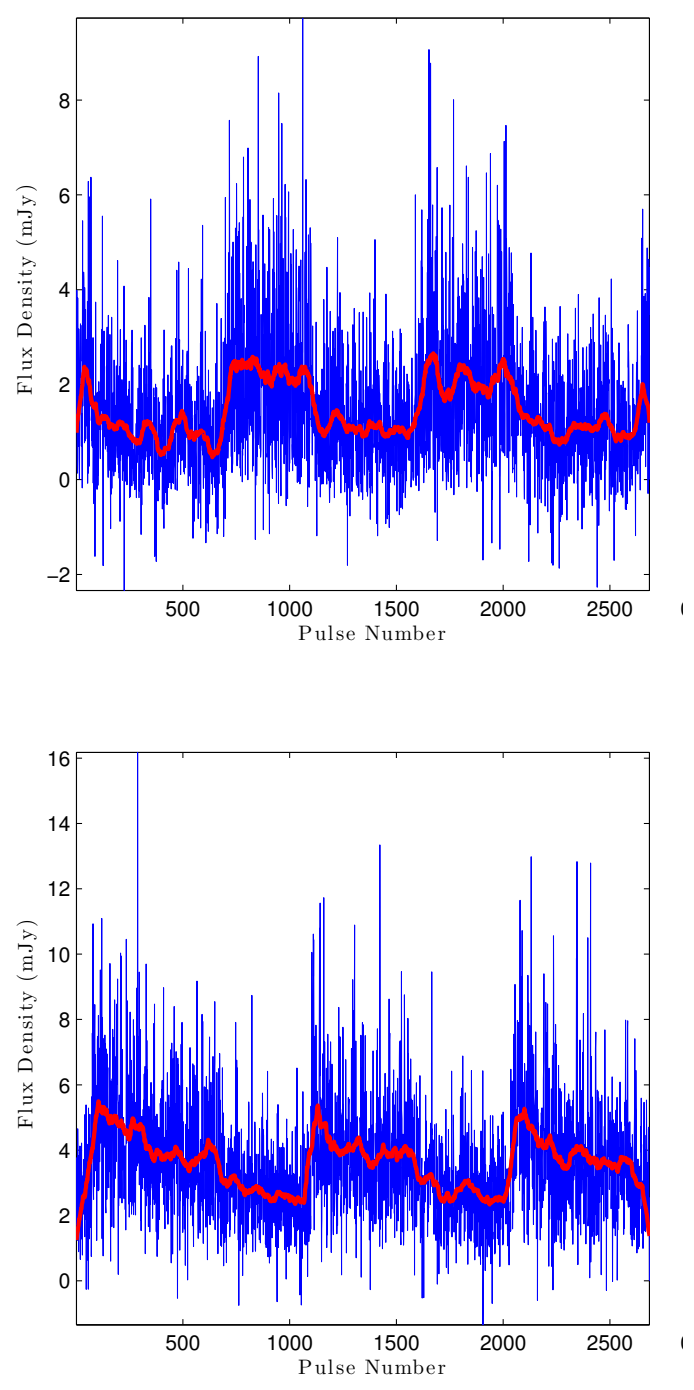

ternate abruptly with those of the right shown in bottom panel of Fig. 2.4. Together these form the undulating pattern in Fig. 2.3.

Figure 2.4: A 15 minute $1.4 \mathrm{GHz}$ observation of B0611+22 on MJD 54898. (Top): The flux within a bin where the left-sided fluctuations dominates in Fig. 2.3. (Bottom): The flux within a bin where the right sided fluctuations dominates. The solid line in both is the boxcar convolution of the time series. Note that flux densities changes alternate between the left and right sided bins 


\subsubsection{Gaussian fitting and skewness measurements}

To quantify the bursting phenomenon, knowing that the pulse is well described by a Gaussian (Weisberg et al., 1999), we fit Gaussians to each profile on data values greater than $25 \%$ of the maximum value of each local mean pulse. This is done to ensure that the fit adheres to the peak of the pulse, and to reduce any asymmetric influences. The resulting fit parameters (peak location $\mu$, amplitude $A$, and pulse width $\sigma$ ) of each pulse can then be compared to see if changes occur between the two emission modes.

To quantify any asymmetry in the data, we measured the skewness of each pulse. This procedure is outlined in Appendix A. In this calculation, to avoid contamination by noise, only flux density levels greater than $5 \%$ of the maximum pulse value are used.

\subsubsection{Gaussian and skewness results}

Note that, due to the smoothing of the data, any structures on scales smaller than 64 pulse numbers in these results must be considered as a noise feature. For this reason, histograms are generated for each parameter value in order to investigate broader statistical trends.

The results from this analysis of the $327 \mathrm{MHz}$ data are shown in Fig. 2.5. There, a clear bimodal distribution is seen in the peak locations $(\mu)$ histogram. These values are then plotted against pulse number, the lower graph in the $\mu$ column of Fig. 2.5. 

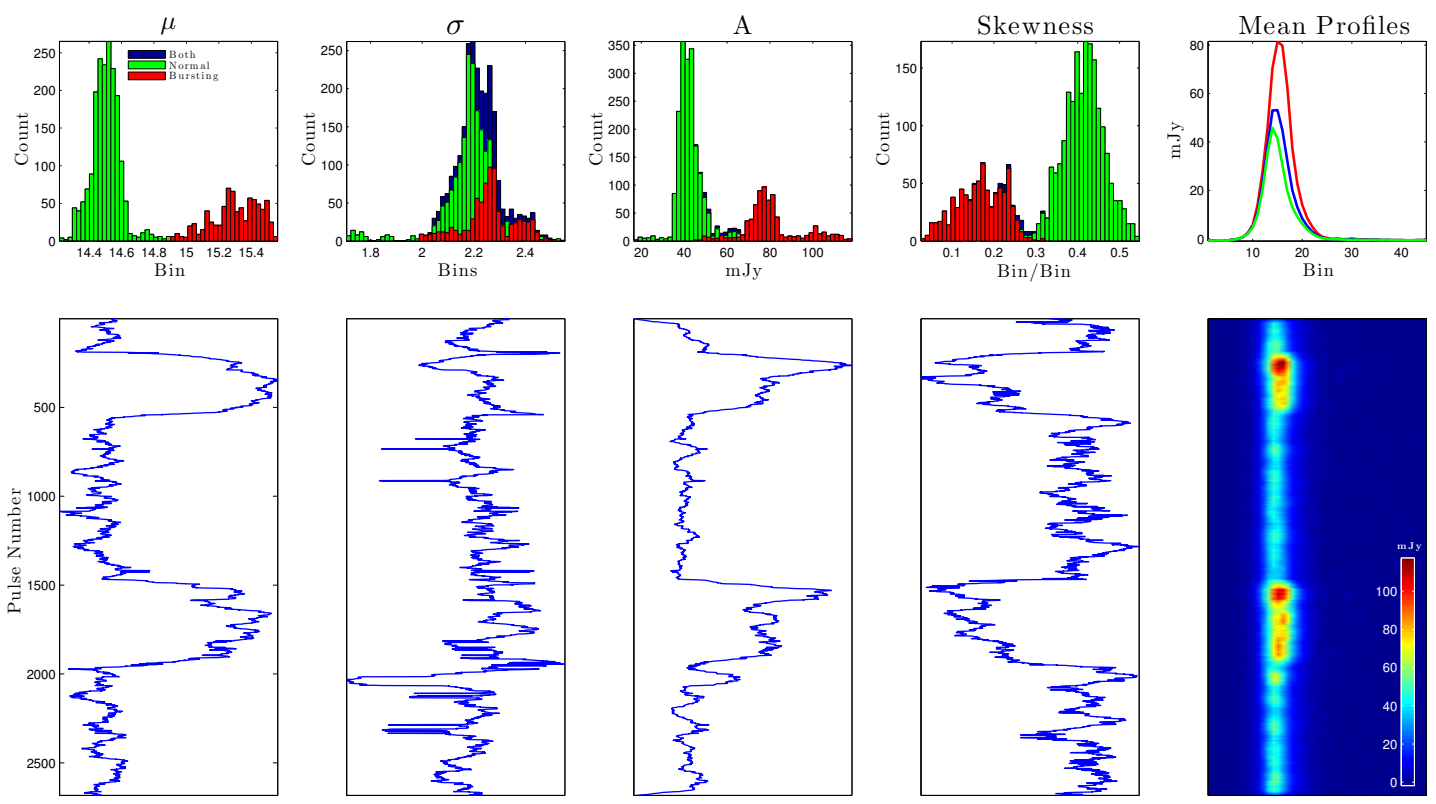

Figure 2.5: Gaussian parameters and skewness measurements of the smoothed data from the observation carried out on MJD 54898 at $327 \mathrm{MHz}$.

It then quickly becomes evident that the larger values of $\mu$ are associated with the bursting pulses. This directly conveys a phase-shift between the two emission modes.

To isolate the two populations, we set a threshold around the midpoint (at bin 14.9) on the $\mu$ values. Pulse numbers with $\mu$ values below this threshold are labeled as a normal pulse, and the rest are labeled as a bursting pulse. From this indexing, subset histograms are formed for the other recorded parameter values to see how these emission modes contribute to each distribution.

For the pulse width $(\sigma)$ values measured in the $327 \mathrm{MHz}$ data, there is little that distinguishes the two modes, while the amplitude values $(A)$ are dominated at the higher end by the bursting mode. This is congruent to what was observed in the smoothed data set for this frequency, seen in Fig. 2.1 and again in the far right of Fig. 2.5. When these amplitude values are plotted against the pulse number, the 

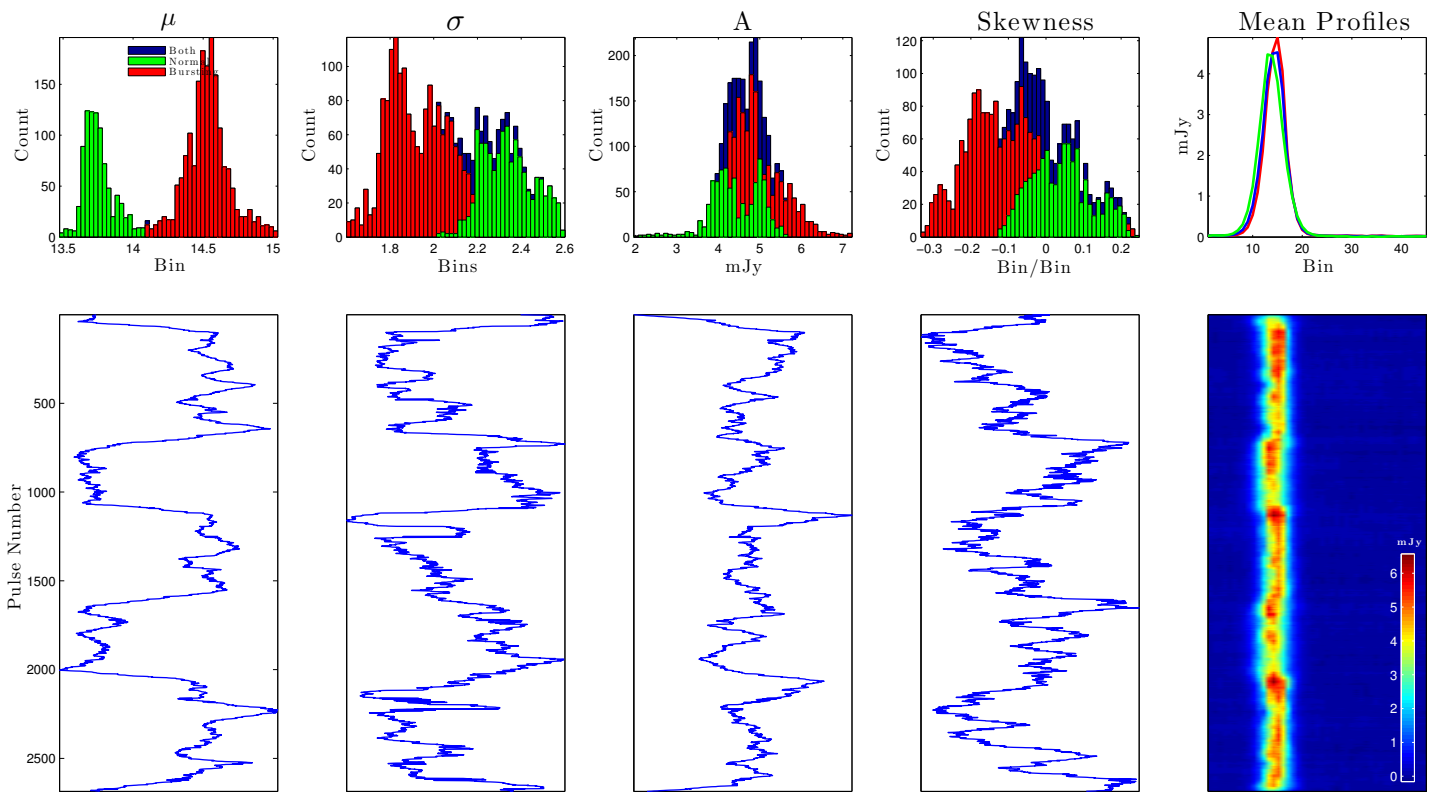

Figure 2.6: Gaussian parameters and skewness measurements of the smoothed data from the observation carried out on MJD 54898 at $1.4 \mathrm{GHz}$.

lower graph in the $A$ column of Fig. 2.5, the decaying behavior mimics what was seen in Fig. 2.2. This confirms that this decay is an overall effect on the pulse and not a single bin phenomenon. Skewness measurements at $327 \mathrm{MHz}$ show a clear separation between the two modes, and the bursting pulses tend to have a lower skewness than the normal mode.

We also wish to see if these trends are noticeable in the pulse profiles. To do this, pulses from the raw data were averaged over the pulse numbers of the corresponding modes and over the whole observation for comparison. These profiles are shown in Fig. 2.5. There we can see that the mean bursting pulse is indeed larger in amplitude, comparable in width, and its peak is slightly shifted compared to the mean normal pulse. 

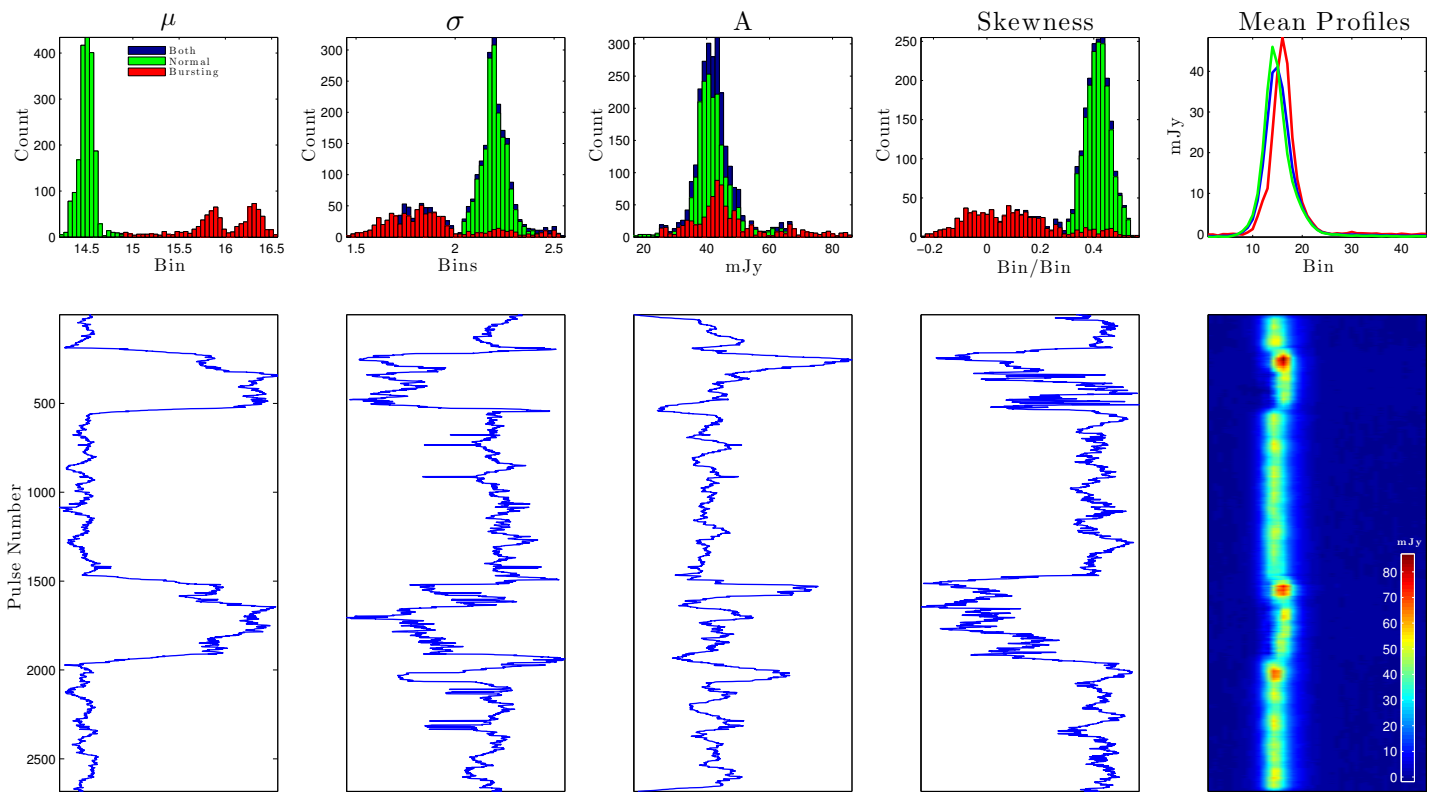

Figure 2.7: Gaussian parameters and skewness measurements of the smoothed data from the observation carried out on MJD 54898 at $327 \mathrm{MHz}$ with the normal profile removed from the bursting modes.

To see if these trends are consistent at other frequencies, the analysis was performed on the 1.4 GHz observation from the same day, shown in Fig. 2.6. Again we see a clear bimodal distribution in the $\mu$ values. When plotted against pulse number, a noticeable phase shift is seen that corresponds with the undulation observed in the smoothed perturbations of Fig. 2.3. Again, a $\mu$ threshold about the midpoint (at bin 14.1) was set to isolate the two modes for comparison.

When these $\sigma$ values are compared, it is seen that the bursting pulses tend to be narrower than the normal pulses. This trend was not seen in the $327 \mathrm{MHz}$ data, along with little distinction in the amplitude. On the other hand, the bursting pulses still have the tendency towards lower skewness values.

In efforts to explain these varying trends, the $327 \mathrm{MHz}$ observation is reexamined. The larger amplitude and similar width of the bursting pulse allows for 
a nested normal pulse to be simultaneously emitting. From the indexing of the previous analysis, we remove the mean normal pulse for the bursting sequences in the raw dataset. This new set is then convolved and Gaussian fitted to form Fig. 2.7. There we can see that the new trends reflect the ones observed at $1.4 \mathrm{GHz}$, where the bursting pulses are narrower than the normal mode and the amplitudes are of similar magnitude.

Mean profiles for each situation are presented in the far right hand corners of Fig. 2.5, 2.6, and 2.7. The bursting profiles for each are contained within the normal profile's starting and ending envelope. Therefore, it appears that the different emission mechanisms are confined to a single emission cone. This confinement is also supported by the skewness measurements, where the asymmetry of the profile is changing to accommodate for this restraint, as well as the phase changes.

\subsubsection{Bursting rate}

When the analysis from the previous section is performed on the other observations from Table 2.1, we find that bursting events are occurring rather frequently in this pulsar, with at least one mode shift per observation. All the observations support the same statistical trends in Fig. 2.5 and 2.6 for their respective bands. From the length of each burst and the total number of events over all the observations, we estimate a bursting event occurs approximately every seven minutes (1200 pulses) lasting for two to four minutes (300 - 600 pulses). 

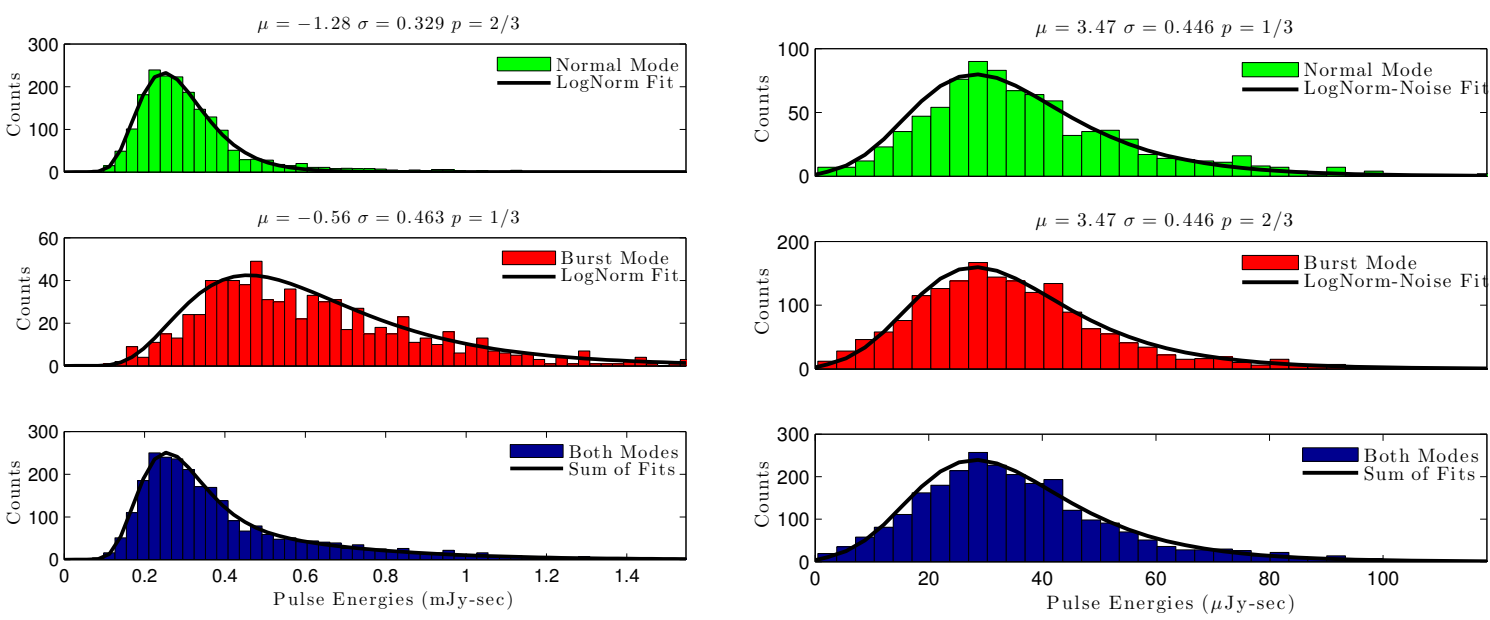

(a)

(b)

Figure 2.8: Pulse energy distributions for each mode and for entire observation.(a) The distributions for the $327 \mathrm{MHz}$ observation on MJD 54898 with log-normal fits. (b) The distributions for the $1400 \mathrm{MHz}$ observation on MJD 54898 with log-normal affected by noise fits.

\subsubsection{Pulse energy distributions}

From the indexing in the preceding sections, we are able to form subset pulse energy distributions for each mode. This was achieved by summing ten phase bins on either side of the maximum mean phase bin for each pulse in the raw data sets. A bin span was then converted to time and multiplied by the sum to produce an energy for each pulse. The energy distributions for each mode and the overall observation are then generated, see Fig. 2.8.

For the $327 \mathrm{MHz}$ pulse energy distributions, a log-normal probability density function $(\mathrm{PDF})$ is fitted for each mode with a maximum-likelihood estimation (mle). From these fits, shown in the left side of Fig. 2.8, both modes are will described by different log-normal distributions occupying different percentages $(p)$ of the overall observation. Here we again see that the bursting pulses are more energetic, dom- 
inating the higher energies while the normal mode pulses are centralized at lower energies. When these two fits are summed and compared to the overall energy distribution, seen in the lowest left panel in Fig. 2.8, we can see that it matches very well to support that our indexing separates these two modes effectively.

When the same method was applied to the $1400 \mathrm{MHz}$ observation, the lognormal fits did not provide a satisfactory fit to the data in the lower energy range. Because of this and the overall lower energy levels, we wanted to investigate whether the noise was affecting an underlying log-normal distribution. To incorporate this noise to a log-normal PDF, we needed to integrate the probability of other values $\left(x^{\prime}\right)$ being read at another location $(x)$ due to the Gaussian noise. The resulting PDF is

$$
P D F(x)=\int_{0}^{\infty} \frac{1}{x^{\prime} \sqrt{2 \pi \sigma^{2}}} \mathrm{e}^{\frac{-\left(\ln x^{\prime}-\mu\right)^{2}}{2 \sigma^{2}}} \frac{1}{\sqrt{2 \pi \sigma_{n}^{2}}} \mathrm{e}^{\frac{-\left(x-x^{\prime}\right)^{2}}{2 \sigma_{n}^{2}}} \mathrm{~d} x^{\prime}
$$

where $\mu$ and $\sigma$ are the shape parameters for the underlying log-normal and $\sigma_{n}$ is the standard deviation of the Gaussian noise. Because of the symmetry of the Gaussian, this is the same as convolving the log-normal with a normal distribution.

To find a value for $\sigma_{n}$, we summed and converted 21 bins in the off-pulse region, then fitted a Gaussian to this energy distribution, shown in Fig. 2.9. We repeatedly found that the energies in the off-pulse regions were well described with $\sigma_{n}=6.43$ $\mu \mathrm{Jy}-\mathrm{sec}$.

Unfortunately, the PDF in Eq. 2.1 has no analytical solution. Therefore, we approximate this with a finite intervals summation and use these values as our new PDF for the maximum-likelihood estimation fitting. These fits matched the distribu- 
tion remarkably well, now describing the whole distribution with little change in the log-normal shape parameters, shown in the right side of Fig. 2.8.

There we can see that both modes

can be described as a single energy distri-

bution. This supports what was seen in

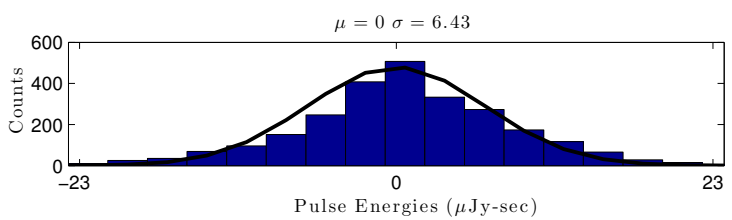

the previous sections, that there was lit-

tle change in the amplitudes of the pulses

Figure 2.9: Off pulse energy distribution with a Gaussian fit.

between the two modes. What is peculiar is that the pulse width changes between the modes are not significant enough to be reflected in the energies. This may be because of the noise contribution.

\subsection{Sub-pulse drifting analysis}

We now take the opportunity to revisit the issue of the presence of any sub-pulse drifts in the two emission states of B0611+22. If sub-pulse drifts are occurring with regular frequency, there should be a dominant peak in the Fourier transform of each phase bin of the raw data (Backer, 1970a,b). While this analysis has been performed on this pulsar before in Weltevrede et al. $(2006,2007)$ with no features found, the two modes have not been looked at independently. Therefore, we investigate each mode separately to see if any differences can be seen in the frequency domain. Because we are just interested in the fluctuations of the profile, the mean value of each phase bin is subtracted before calculating a fluctuation spectra. 


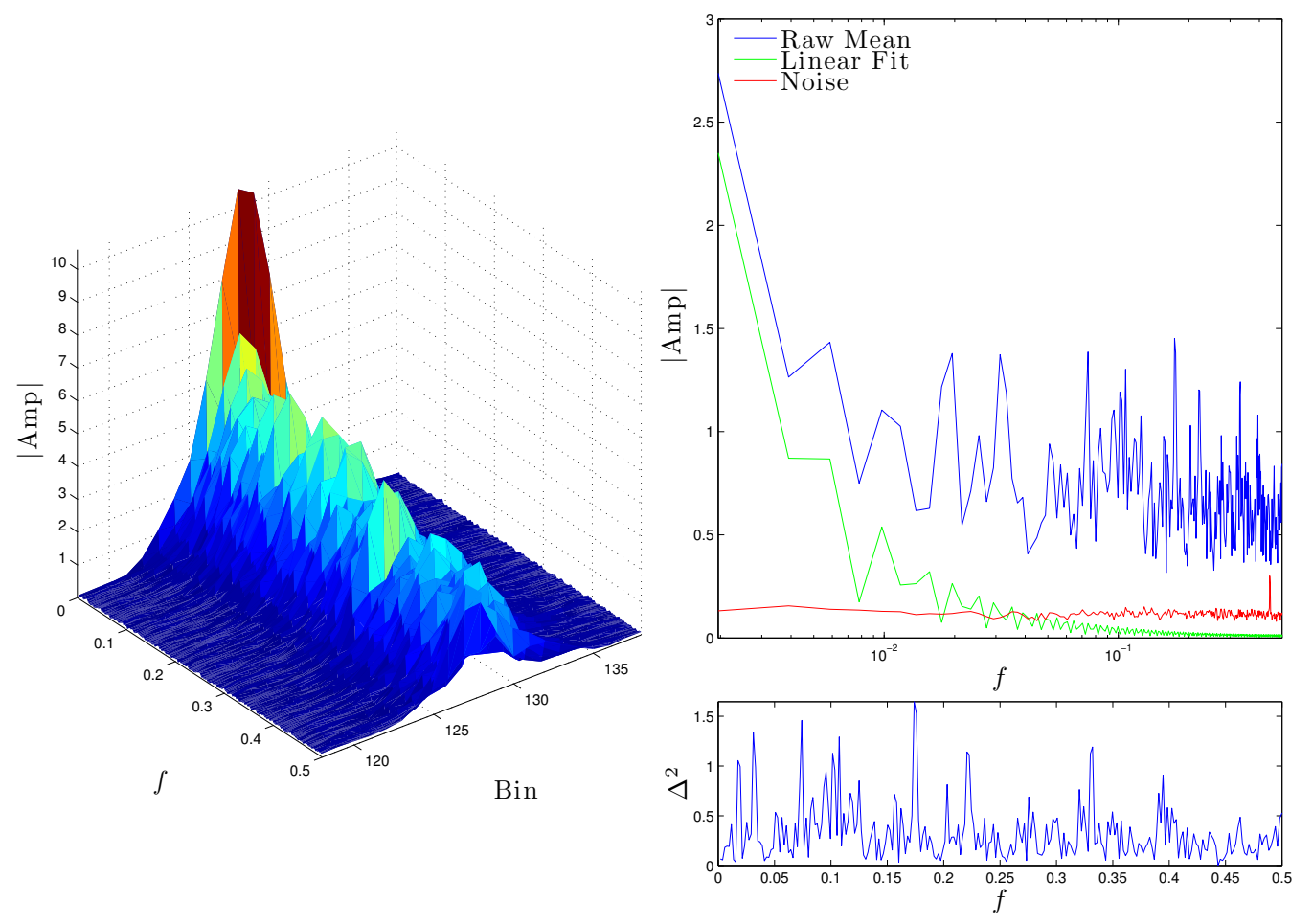

Figure 2.10: (Left) The fluctuation spectra of the first bursting event for $327 \mathrm{MHz}$ in Fig. 2.1, covering 21 bins centered on the mean peak and from pulse-numbers 220 to 530. (Upper right) The mean amplitude of each frequency in the Left figure. Along with the Fourier transform of a linear fit of the mean flux from the same section in Fig. 2.1, and the mean amplitude of frequencies from an off pulse area of the same size. (Lower right) The square of the difference of raw mean amplitude from the amplitudes of the linear fit and the mean off source. Here all frequencies $(f)$ are in units of cycles-per-period $(\mathrm{C} / \mathrm{P})$. 


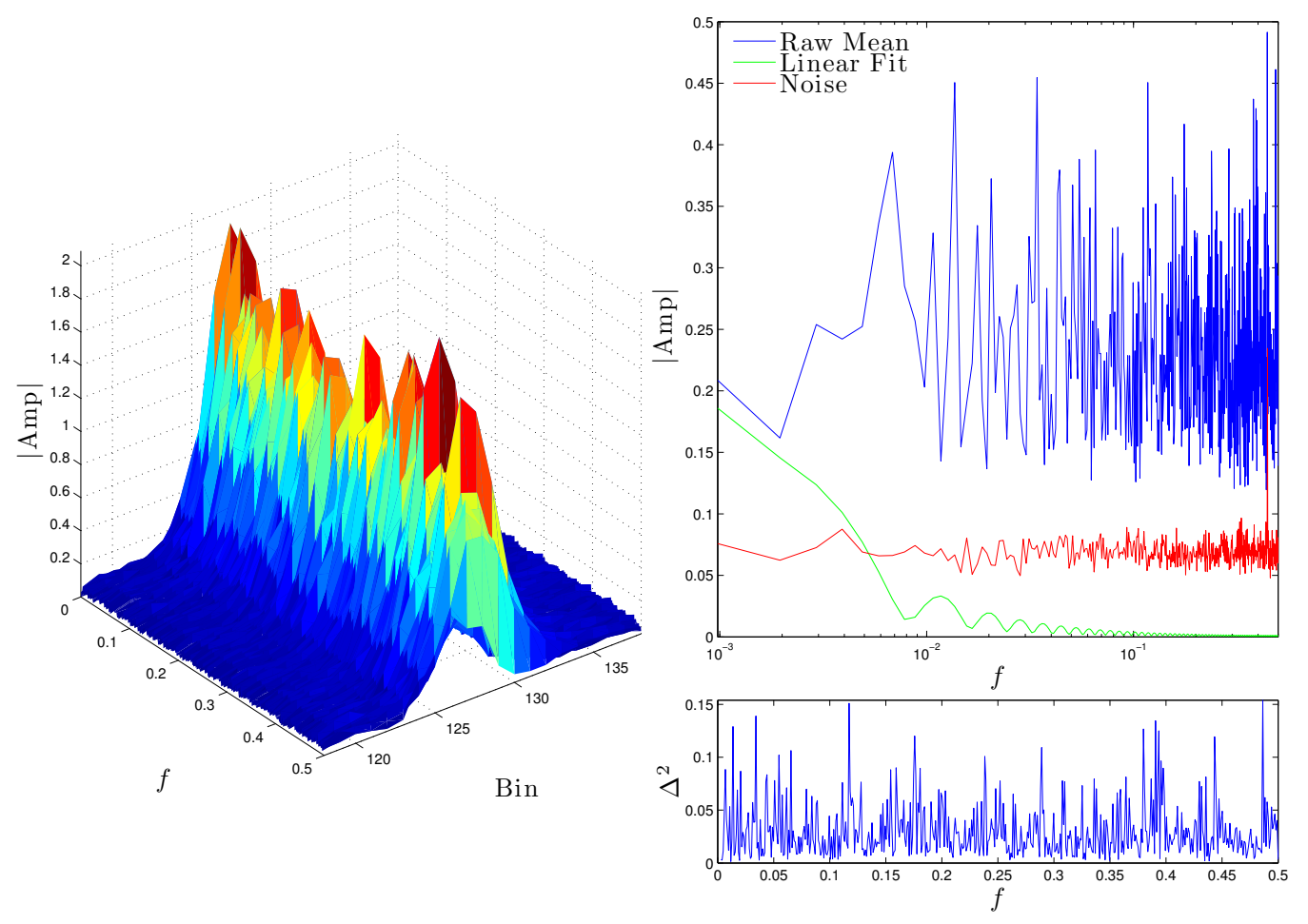

Figure 2.11: (Left) The fluctuation spectra of the longest normal mode for $327 \mathrm{MHz}$ in Fig. 2.1, covering 21 bins centered on the mean peak and from pulse-numbers 550 to 1450. (Upper right) The mean amplitude of each frequency in the Left figure. Along with the Fourier transform of a linear fit of the mean flux from the same section in Fig. 2.1, and the mean amplitude of frequencies from an off pulse area of the same size. (Lower right) The square of the difference of raw mean amplitude from the amplitudes of the linear fit and the mean off source. Here all frequencies $(f)$ are in units of cycles-per-period $(\mathrm{C} / \mathrm{P})$. 
When investigating amplitudes of the first bursting event at $327 \mathrm{MHz}$, shown in the left hand side of Fig. 2.10, it soon becomes evident that the lower frequencies are playing a dominant role. To examine why this is, we first produce a linear fit of the mean intensities of each pulse number. This fit is then Fourier transformed and compared to the the mean amplitude of each frequency in the data, shown in the upper right hand side of Fig. 2.10. We can then see that in the low frequency range these two spectra are comparable. This suggests that these low frequencies are a consequence of the large scale structure of the bursting envelope.

To see if any radio interference is contributing, the fluctuation spectra of an off-pulse region of the same area are calculated for comparison. The mean value of this region is plotted in the upper right hand side of Fig. 2.10, where a single signal is seen at high frequency. This radio interference does not appear to be a major contributor. Regardless, this and the linear trend spectra are subtracted from the mean data spectra. This difference in amplitudes is then squared to approximate how the other components are contributing to the power, seen in the lower right of Fig. 2.10. There we can see that there are no substantial single frequency peaks, suggesting that sub-pulse drifting is not prevalent in this mode. When the same procedure is preformed on the normal mode (Fig. 2.11), there is clearly no low frequency dominance as what was seen in the bursting mode. This is consistent with the structural explanation since the normal mode should have no large-scale pattern. Again we see no prominent peaks in the amplitude difference.

These results are in agreement with what was reported in Weltevrede et al. (2006, 2007). This analysis does suggest however, that if a 'red noise' component 
exists in the fluctuation spectra it may be a sign of intrinsic structures in the intensity and should not always be disregarded as interstellar scintillation or due to receiver fluctuations (Lorimer \& Kramer, 2005).

\subsection{Conclusion}

In summary, using a sensitive boxcar convolution method to preserve the phase resolution, we have found exceptional pulse patterns in the radio emissions from B0611 +22 in archival data taken with the Arecibo radio telescope.

With this analysis, prolonged enhanced emissions were discovered at $327 \mathrm{MHz}$. When investigating the overall structure of this emission, it was shown that these are bursting events that abruptly appear and then systematically decay. These events appear to be superimposed upon a continual emission mode offset in pulse phase. This was unexpected and is a completely undocumented relationship for this pulsar.

To gain further insight into these events, we searched for them at $1.4 \mathrm{GHz}$ using the same method, finding a different picture as compared to $327 \mathrm{MHz}$. At $1.4 \mathrm{GHz}$, the bursting events were no longer bright episodes, and the steady emission mode was no longer present. At this frequency B0611+22 appears to be a mode switching pulsar, where one mode is the decaying burst and the other mode is constant when on. This mode switching is surprising in this pulsar, only being suggested briefly in the past (Nowakowski, 1992; Nowakowski \& Rivera, 2000). B0611+22's characteristic age is also surprising for this behavior, because the majority of mode-switching pulsars are a couple of orders of magnitude older (Wang et al., 2007). This discrepancy may 
suggest that bursting pulsars are a new classification of pulsars that are independent of nulling and moding.

To investigate these relationships, we fitted Gaussians and measured the skewness of each convolved pulse. These measurements enabled us to confirm that there was a phase change between the two modes. This was utilized to isolate each of the mode statistics in each frequency range. We then showed that the parameter differences were reflected in each mode's mean profile. These mean profiles, along with the skewness measurements, support that the modes are confined to the same phase region and therefore the same emission cone.

This analysis also shows that, apart from the amplitude difference from 327 to $1400 \mathrm{MHz}$, there was also a change in the distribution of the pulse-width parameters. To see what could be causing this distribution change, we revisited the $327 \mathrm{MHz}$ data and subtracted the constant underlying emission from the burst. When this was done, the normal mean profile from the $327 \mathrm{MHz}$ bursting event distributions became consistent with the $1.4 \mathrm{GHz}$ distributions.

An investigation of the pulse energy distributions also revealed differences between the two modes. While both modes can be described by log-normal probability density functions, at $327 \mathrm{MHz}$ the modes are independent, but at $1400 \mathrm{MHz}$ both mode energies are in a single function.

The two modes were also searched for drifting sub-pulses independently, where no significant signals were present in any of the fluctuation spectra, supporting Weltevrede et al. $(2006,2007)$ that sub-pulses are not prevalent in this pulsar. 
Before this analysis, the curious emission properties of B0611+22 were not well known (Nowakowski 1992). Because this is the first pulsar that we have studied with this analysis, this may be a more common occurrence than we realize. In particular this work required the unique sensitivity of the Arecibo telescope to reveal this behavior. Additional studies of this kind are clearly necessary.

On a more global scale, studies such as this can help draw relationships between different types of modal and emission variations. Here it is not difficult to imagine a threshold situation that can relate nulling and bursting events. Consider, for example, similar pulsars to B0611+22 that are either not as bright or are farther away such that the normal mode is undetected. In this case the normal mode would be interpreted as being in a null state. A similar argument was made for B0656+14 in the context of a possible connection with rotating radio transients (Weltevrede et al., 2011). Additional examples of this phenomenon will allow us to build up a more complete picture of the complex emission phenomenology of radio pulsars. 


\section{Chapter 3}

A search for an underlying normal mode in J1752+2359.

Of the three known, bursting pulsars discussed so far J1752+2359 is the only one that is not accompanied by persistent emission between bursting events (Lorimer et al., 2013; Seymour \& Lorimer, 2013). Because of this we explored the possibility of an underlying normal mode that may have been buried in the system noise. How this was performed and the implications are described in this chapter in the following way: in Section 3.1 we provide technical information about the observations; in Section 3.2 we explore the pulse energy distribution; in Section 3.3 we investigate the normal mode using positive number statistics; in Section 3.4 we compare J1752+2359's fluctuation spectrum to other known spectra of bursting pulsars; finally, in Section 3.5, we discuss our results and their scientific significance.

\subsection{Observations}

The data presented here were collected November 27, 2009 (MJD 55162) at a $327 \mathrm{MHz}$ for J1752+2359 using the Wide Band Arecibo Pulsar Processors (WAPPs; Dowd et al., 2000). The data were then converted to flux-density using the radiometer equation (see Lorimer \& Kramer, 2005) by combining both polarizations, using a system temperature of $122 \mathrm{~K}$, a gain of $10 \mathrm{~K} / \mathrm{Jy}$, and a frequency range of $50 \mathrm{MHz}$. 


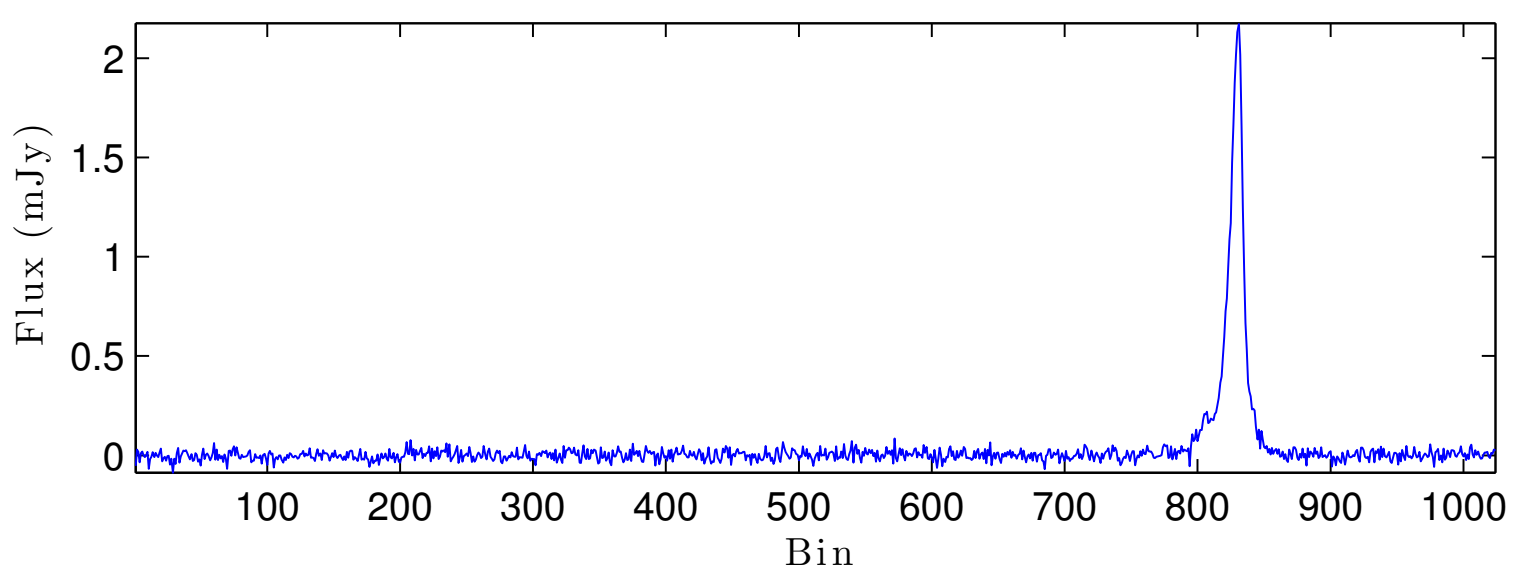

Figure 3.1: The mean pulse profile of the whole observation.

\subsection{Pulse energy distribution}

We first generated the mean pulse of the whole observation. This was simply done by integrating the phase bins of the folded time series, i.e. adding the bins with the same pulse longitude in the successive pulses so that the average intensity $\mu_{i}$ in bin $i$, is

$$
\mu_{i}=\frac{1}{N} \sum_{j=0}^{N-1} A_{i j} .
$$

$N$ is the total number of pulses, and $A_{i j}$ is the flux at pulse longitude bin $i$ and pulse number $j$ in the time series.

From this integrated profile we were able to pick a 71 phase bin region from bin 795 to bin 865 , where the mean pulse appeared to begin and end. To calculate the pulse energy, we summed across this region for each pulse and converted a single bin length to time in milli-seconds. Multiplying the summed flux for each pulse by the bin length time scale produces a pulse energy in micro-Jansky-seconds ( $\mu \mathrm{Jy}$-s). 

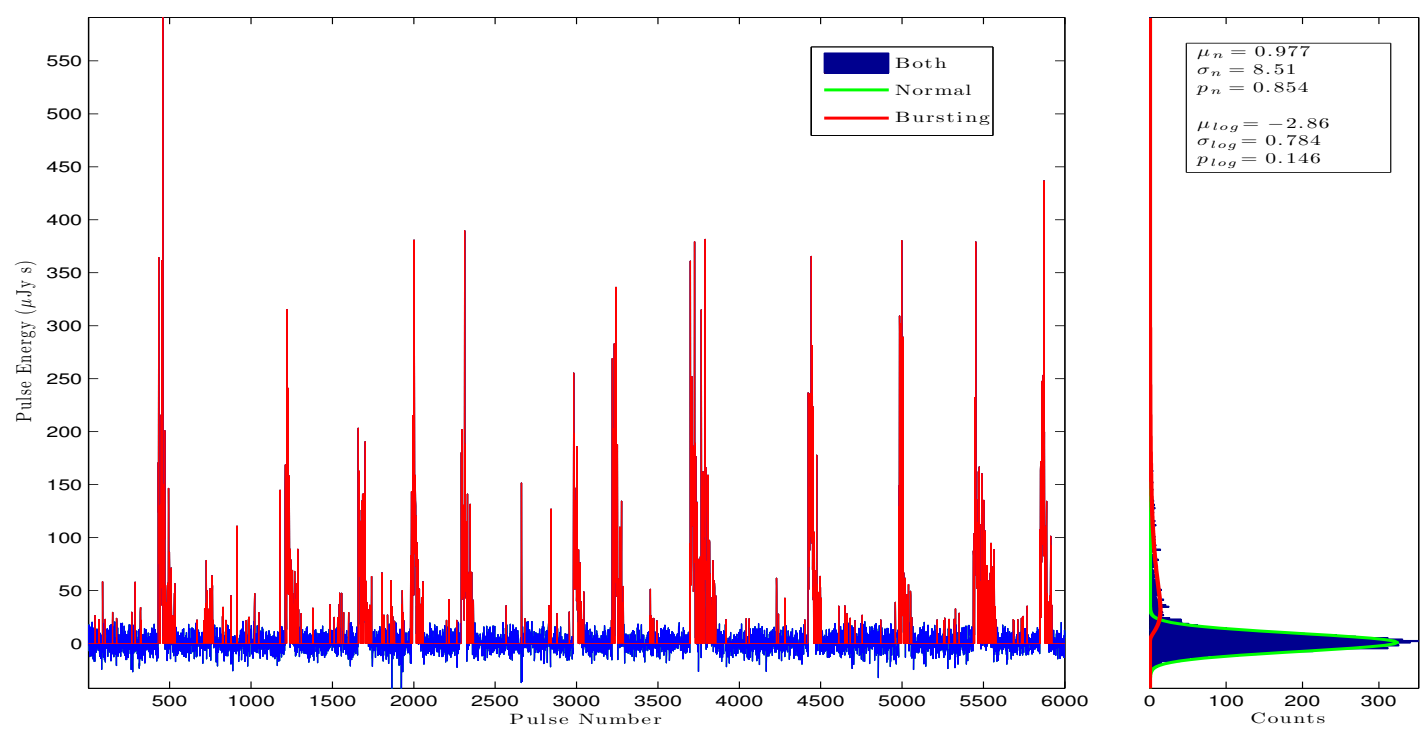

Figure 3.2: Energy values for J1752+2359. (Left) The energy values versus pulse number. The pulses marked in red have an energy greater that $21 \mu \mathrm{Jy}-\mathrm{s}$, this includes most of the bursting and large single pulses. (Right) The energy distribution with a log-normal and normal bimodal fit.

From these values, shown in the left side of Fig. 3.2, it first appears that the bursting events are the dominant features.

The pulse energy distribution, seen in the right of Fig. 3.2, is best described by a combination of a log-normal and normal distributions. There we can see the bursting distribution only contains for about $P_{\log } \sim 15 \%$ of all the pulses. The other $85 \%$ of the pulses have been seen by others as nulling because of their low energy level, and being in a Gaussian distribution that has a deviation similar to the offpulse regions. If these regions are true nulling events, they should result in a Gaussian centered about zero. Yet, from the fit we can tell that there is a slight positive offset of $1.0 \mu \mathrm{Jy}-\mathrm{s}$. While this energy level seems trivial, the estimate of its uncertainty, $\frac{\sigma_{n}}{\sqrt{0.85 * 6000}}= \pm 0.1 \mu \mathrm{Jy}-\mathrm{s}$, suggests that it is significant. 

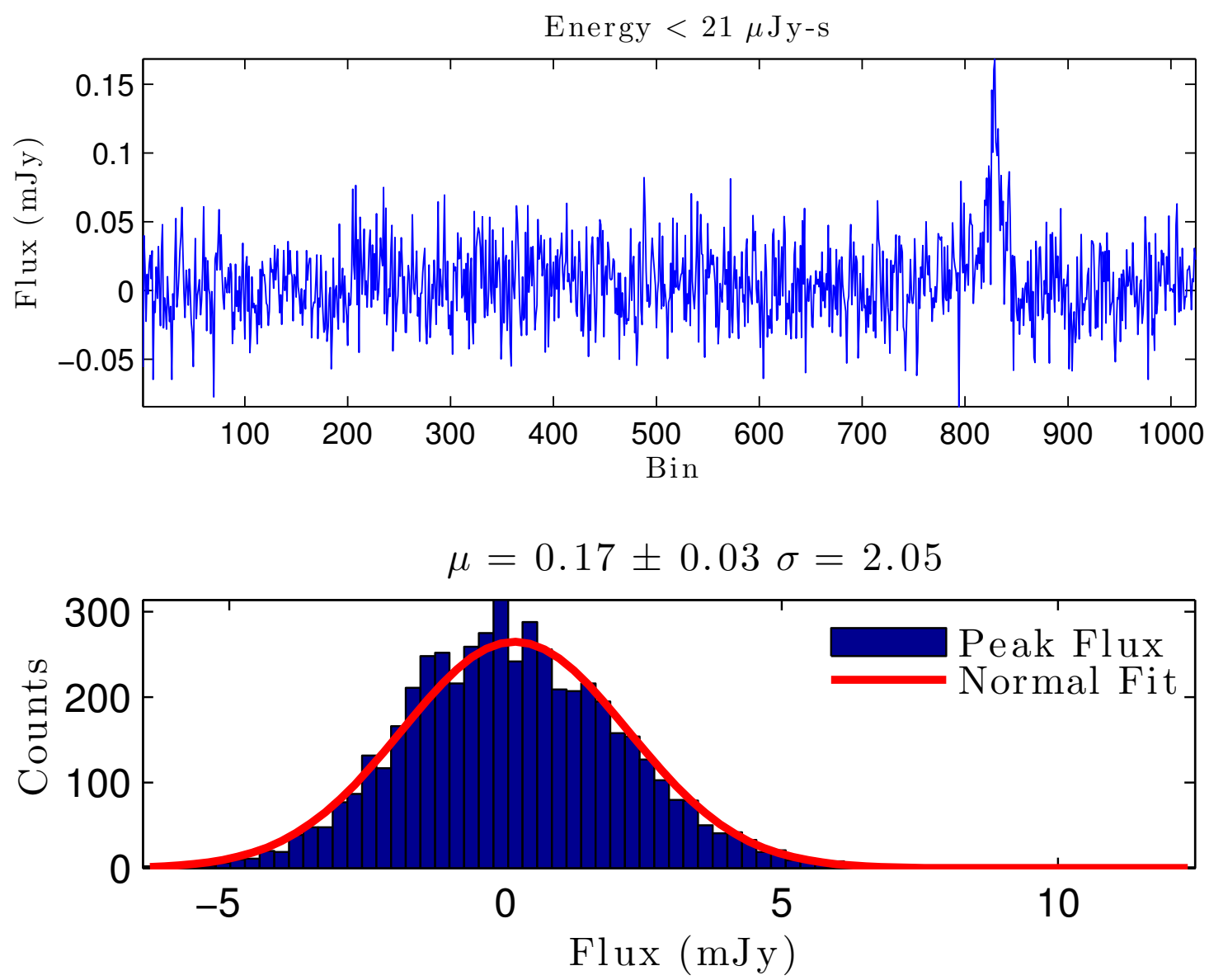

Figure 3.3: (Top) The low energy level mean profile with a pulse-energy the of 0.977 $\mu \mathrm{Jy}-\mathrm{s}$. (Bottom) The distribution of the flux values that resulted in the peak mean flux in upper panel.

Such an offset would imply a low-level normal emission mode and should produce a mean profile when a large number of these pulses are averaged together. In order to include as many pulses as possible without being overly influenced by the pulses in the other distribution, we decided to keep values where the Gaussian distribution was at least one and a half times more likely to occur than the log-normal distribution. This resulted in a pulse energy threshold around $21 \mu \mathrm{Jy}-\mathrm{s}$, and as seen in the left of Fig. 3.2 it appears to exclude most of the bursting and large single pulses. 
When these low level pulses were averaged together, it produced a pulse profile, as seen in the upper panel of Fig. 3.3, with a pulse-energy of the expected value. To

ensure that this profile is not a consequence of a few extreme events, we examined the flux values that resulted in the peak mean flux. The histogram of these flux values is nearly Gaussian and is centered about the peak flux value as shown in the lower panel Fig. 3.3, with a standard deviation similar to the off-pulse regions. This strongly suggests that there is a low level normal mode that has been buried within the system noise.

\subsection{Positive thinking}

Due to the large variance in the peak mean flux distribution, we investigated further into whether the normal profile is a noise buried mode. If there is a persistently emitting pulse, it should constantly raise the flux values in bins where it occurs. This shift increases the likelihood that a number would be positive. Only looking at the positive number statistics reduces the effects of any outlying events and will tell us if there is a consistently emitting mode.

To do this, we formed a new pulse stack by assigning positive fluxes with a value of one and the remaining fluxes with a value of zero. The average of each bin would be the fraction of positive numbers in that bin, shown in Fig. 3.4. This fraction approximates the probability that a value would be positive within that bin, and from these probabilities we were able to estimate the flux value for each bin. This was achieved by approximating an inverse Gaussian cumulative distribution function, 


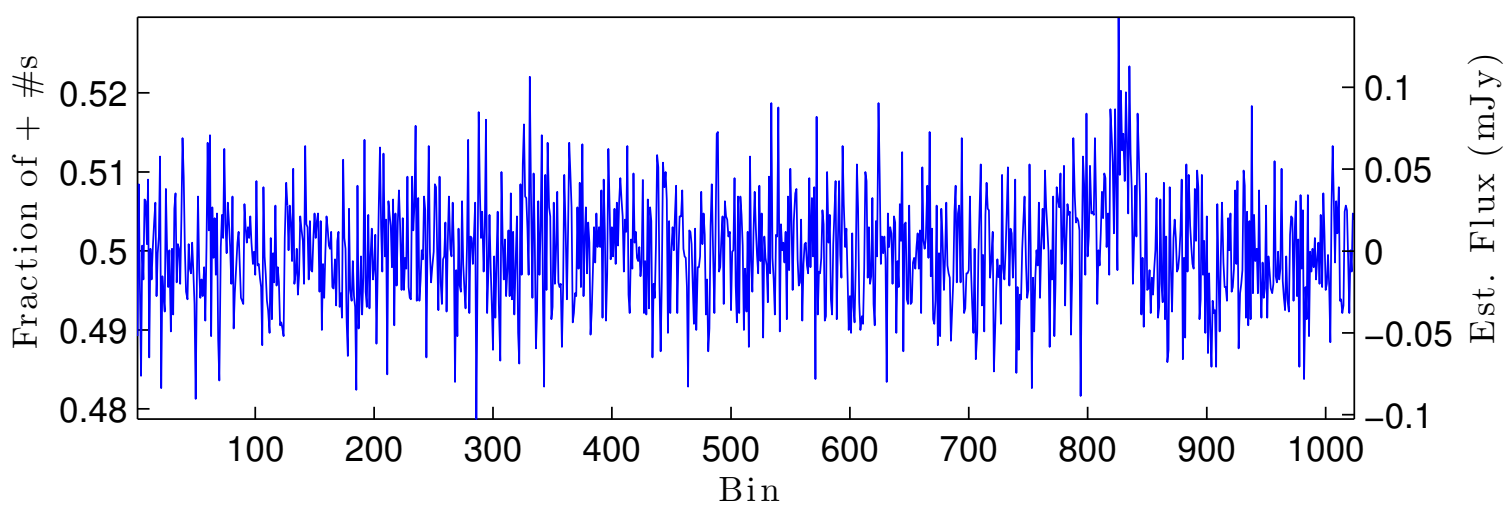

Figure 3.4: The fraction of positive numbers in each bin, along with estimated flux using the positive fraction and assuming Gaussian distribution in each bin with a standard deviation of the noise, $1.924 \mathrm{mJy}$.

also known as the Gaussian quantile function, with a standard deviation of the offpulse region, $1.92 \mathrm{mJy}$.

This method results in a profile with a similar peak flux value as before, seen in Fig. 3.4, supporting that there is a recurring normal pulse but also has increased the noise level. We can understand why this is so if we track the statistics.

We expect a pulse with a peak flux of $0.17 \mathrm{mJy}$ in a noise level of $\sigma_{\text {original }}=$ $1.92 \mathrm{mJy}$, but the noise level should drop as the square-root of the number of pulses averaged over, $N$. This produces an original signal-to-noise ratio of

$$
\frac{S}{N}_{\text {original }}=\frac{(0.17)}{\left(\frac{1.92}{\sqrt{N}}\right)}=0.09 \sqrt{N}
$$

This $0.09 \sigma$ shift translates to a positive value probability of 0.536 and since the noise should be centered on zero, the probability of a positive number to occur in the noise is $P_{\text {noise }}=0.5$. Because the only values we have are zero and one, this probability 
level will have a standard deviation of

$$
\sigma_{P_{\text {noise }}}=\sqrt{P_{\text {noise }}-P_{\text {noise }}^{2}}=0.5
$$

From propagation of errors, we can approximate the standard deviation for the estimated noise flux values

$$
\sigma_{\text {flux }}=(1.92 \mathrm{mJy}) \sqrt{2 \pi} \sigma_{P_{\text {noise }}}=2.41 \mathrm{mJy}
$$

This results in a new signal-to-noise ratio of

$$
\bar{S}_{\text {flux }}=\frac{(0.17)}{\left(\frac{2.41}{\sqrt{N}}\right)}=0.07 \sqrt{N},
$$

which is lower than the original in Eq. 3.2.

\subsubsection{Positives in a row}

Looking back at Eq. 3.4 we can see a reduction in $\sigma_{P_{\text {noise }}}$ causes a direct reduction in the deviation of the flux estimate. Believing that we can improve these deviations, we investigated these positive number statistics further.

We can improve the positive probability estimates by looking at how many positive numbers occur in a row. Since each noise data point has a one-half chance of being a positive number, this gives a probability of $P_{\text {noise }}^{n}$ for $n$ positive numbers to 


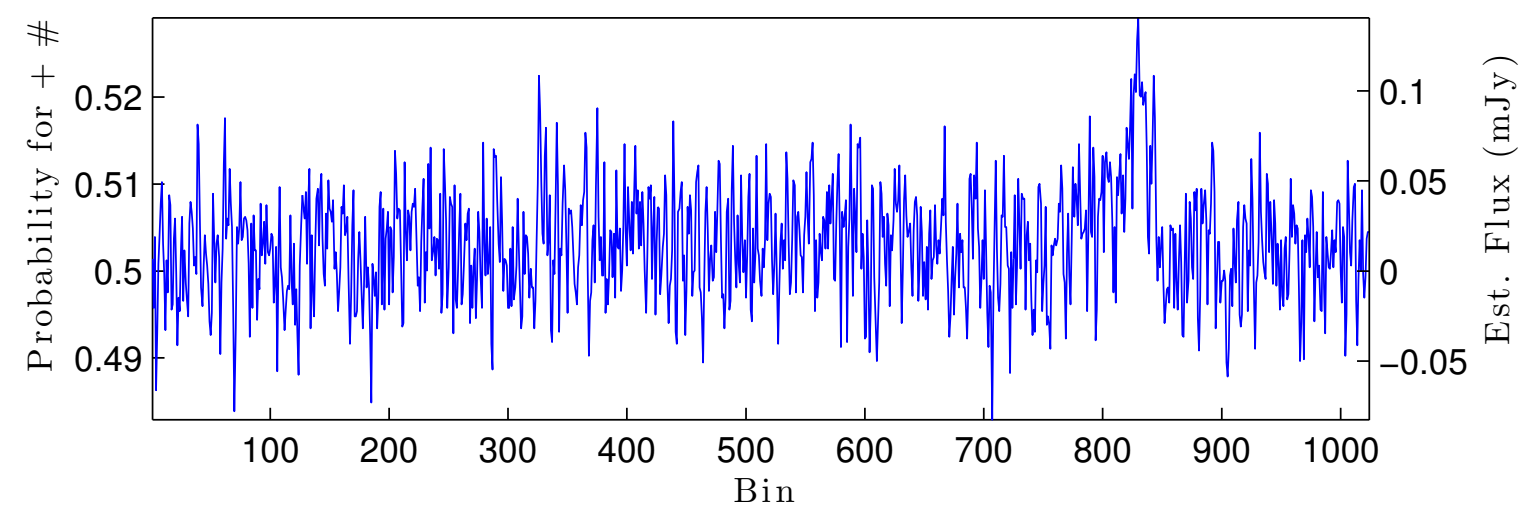

Figure 3.5: Stacked time series where two positive numbers are in a row along pulsenumber.

be in a row. Similar to Eq. 3.3 this new probability will have a standard deviation of

$$
\sigma_{P_{\text {noise }}^{n}}=\sqrt{\left(P_{\text {noise }}^{n}-P_{\text {noise }}^{2 n}\right)} .
$$

Using propagation of errors, we approximate the deviation of the probability estimate

$$
\sigma_{P_{\text {noise }}}=\sigma_{P_{\text {noise }}^{n}}\left(\frac{d P_{\text {noise }}^{n}}{d P_{\text {noise }}}\right)^{-1}=\frac{P_{\text {noise }}}{n} \sqrt{\frac{1}{P_{\text {noise }}^{n}}-1}
$$

Inserting 0.5 for $P_{\text {noise }}$ in the above equation and varying $n$ we find that the deviation is lowest around $n=2$ with a value of $\sigma_{P_{\text {noise }}}=\frac{\sqrt{3}}{4}$. This probability deviation corresponds to a $\sigma_{\text {flux }}=2.09 \mathrm{mJy}$, and gives us a flux signal-to-noise ratio of

$$
\frac{S}{N}_{\text {flux }}=\frac{(0.17)}{\left(\frac{2.09}{\sqrt{N}}\right)}=0.08 \sqrt{N}
$$

To implement this technique, we circularly convolve the binary stack that was created in the previous section with a boxcar kernel spanning two bins along each 
pulse. We assigned each location that had an average less than one as a zero which produces a new binary pulse stack. The mean of each bin in this new stack approximates the positive probability squared; these probabilities are seen in Fig. 3.5. Then we estimated the flux from these probabilities as we did before which resulted in a profile, again seen in Fig. 3.5, with a comparable noise level as the original. In this profile, we again see that there is a positive bias in the pulse bins that correspond with the original flux estimates to further suggest that there is a consistently emitting mode.

\subsubsection{Stacking positive}

We can further improve the probability estimates by summing several positivesin-a-row results. As seen before, we should expect $N P^{i}$ number of events for a starting row size of $i$. When each result is summed, a fraction of $P$ of the highest values is expected to increase by one; this leaves a fraction of $(1-P)$ remaining at the previous value. Summing an infinite number of times results in a noise probability distribution of

$$
P_{n}=P_{\text {noise }}^{i+n-1}\left(1-P_{\text {noise }}\right)
$$

for $n>0$ and will give a noise expectation value of

$$
E=\sum_{n=1}^{\infty} n P_{n}=\frac{P_{\text {noise }}^{i}}{1-P_{\text {noise }}}
$$


This noise distribution will also have a variance of

$$
\sigma_{E}^{2}=\frac{P_{\text {noise }}^{i}}{\left(1-P_{\text {noise }}\right)^{2}}\left[1+P_{\text {noise }}-P_{\text {noise }}^{i}\right]
$$

Again with the aid of propagation of errors, we can find an approximate value of the probability estimate's deviation

$$
\begin{aligned}
\sigma_{P_{\text {noise }}}= & \sigma_{E}\left(\frac{d E}{d P_{\text {noise }}}\right)^{-1} \\
& =\frac{P_{\text {noise }}\left(1-P_{\text {noise }}\right)}{i\left(1-P_{\text {noise }}\right)+P_{\text {noise }}} \sqrt{\frac{1+P_{\text {noise }}}{P_{\text {noise }}^{i}}+1 .}
\end{aligned}
$$

When inserting 0.5 for the probability and varying $i$, we find that the deviation is lowest at $i=1$ with a value of 0.354 . Inserting this value into Eq. 3.4, returns a flux deviation 1.70 mJy. This deviation then results in a signal-to-noise ratio of

$$
\bar{S}_{\text {flux }}=\frac{(0.17)}{\left(\frac{1.70}{\sqrt{N}}\right)}=0.10 \sqrt{N}
$$

This means through this new method, we can improve our detection of the normal pulse by over ten percent.

To execute this technique, we used the method outlined in the previous section to produce a binary pulse stack for each $n$ ranging from one until the final pulse stack was all zeros. Then we summed all these binary stacks together to form a single pulse stack. By averaging along each bin, it returns an estimate for $\frac{P}{(1-P)}$. Then solving for $\mathrm{P}$ and estimating the flux, results in a cleaner profile than the original as predicted and as demonstrated in the bottom of Fig. 3.6. 


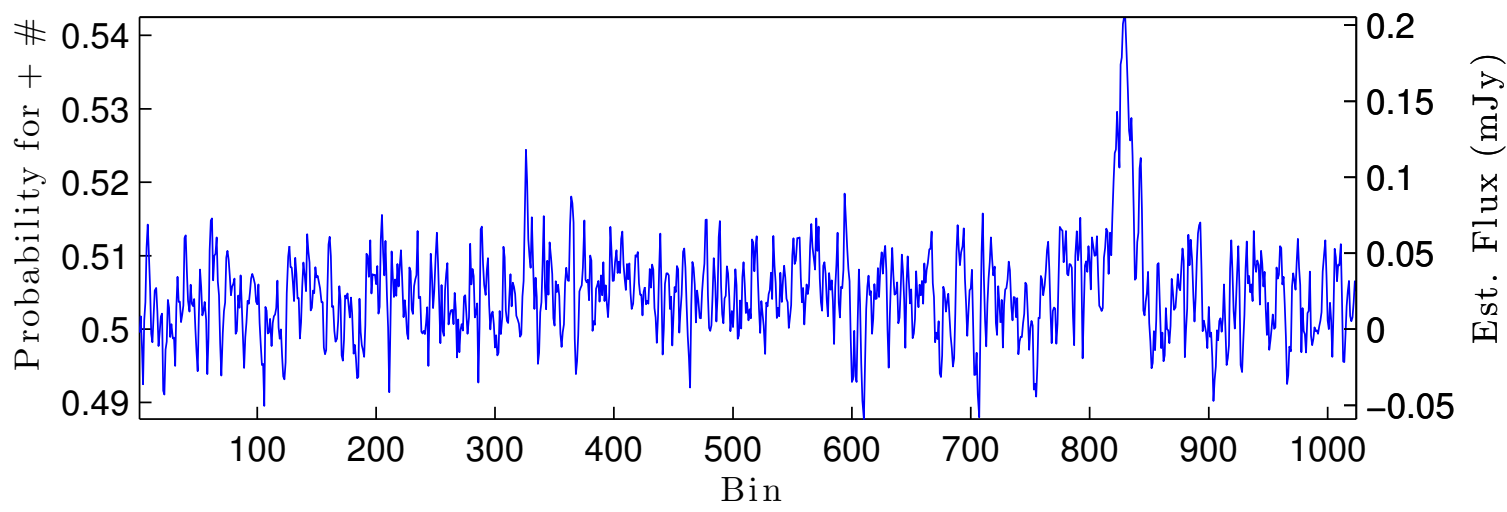

Figure 3.6: Probability of and the estimated flux based on summed positive-in-a-row statistics.

With each positive number statistical method, the peak flux approximation has been within the uncertainty range of the original estimate. Each method supports the notion that there is an underlying consistently emitting normal mode within the noise levels of J1752+2359. This normal mode is congruent with what has been observed in other known bursting pulsars.

\subsection{Sub-pulse drift}

To see if J1752+2359 is consistent in other ways with known bursting pulsars, we searched for sub-pulse drifts. We want to keep the two operation modes separate to study them individually but utilize every event to our benefit.

We first selected a window of 21 phase bins that was centered on the peak of the integrated profile. After this, we first manually picked the start of each burst then generated fluctuation spectrum amplitudes for 21 phase bins that lasted 100 pulses into each bursting event (Backer 1970). It is important to note that all burst- 

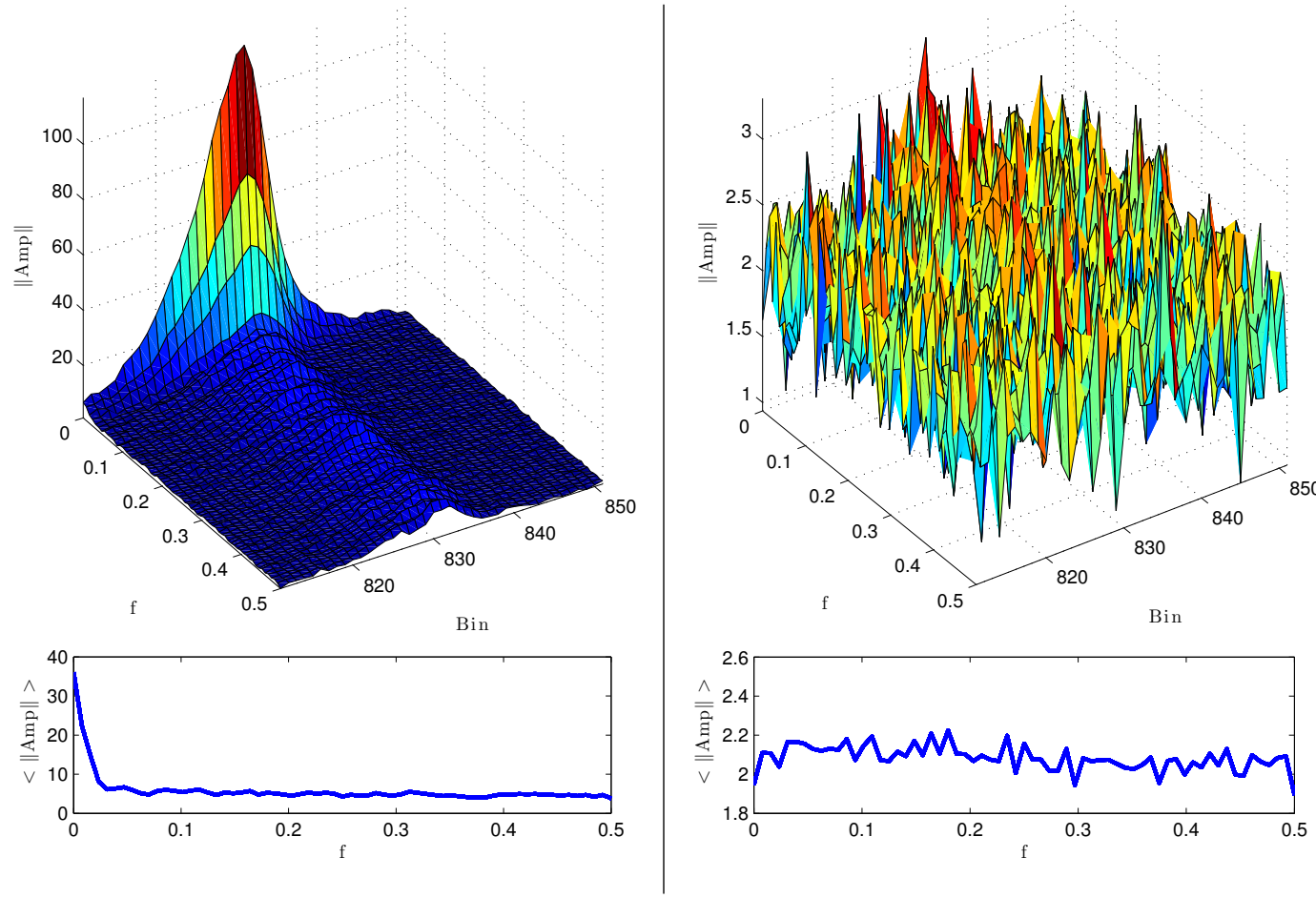

Figure 3.7: Sub-pulse drift analysis.(Top left) The ensemble average of the fluctuation spectrum amplitudes for the bursting events within 810 to 850 bins (see text). (Bottom left) The average frequency amplitudes across the phase bins of the top left figure. (Top right) The ensemble average of the fluctuation spectrum amplitudes for the 'nulling' events within 810 to 850 bins (see text). (Bottom right) The average frequency amplitudes across the phase bins of the top right figure. Here all frequencies $(f)$ are in units of cycles-per-period $(\mathrm{C} / \mathrm{P})$. 
ing events lasted longer than a 100 pulse duration. By picking the same duration, the fluctuation spectrum returns a matrix with the same frequency resolution for each bursting event. This allows each section to be summed together to reduce the noise level and to increase any common frequencies among the events. The summed fluctuation frequencies are shown in the the upper left of Fig. 3.7.

In that figure, the low frequencies dominate the spectrum which arises from the structure of the bursting envelopes. If the pulsar showed a periodic pulse modulation, there would have been an increase of the amplitude at a particular frequency across all phase bins. No such features are immediately visible within this region. To investigate this further, we averaged across the phase bins. This average, shown in the lower left side of Fig. 3.7, uncovered no prominent fluctuation frequencies to suggest no common sub-pulse drifting phenomena within the bursting events. This is consistent with what has been reported for other bursting pulsars.

A similar method was then carried out on the nulling/normal modes. Here the same phase bins were chosen but used 100 pulses just before the start of each bursting event. This resulted in the plot in the upper right panel of Fig. 3.7, where the spectrum appears to be white noise. We again averaged over all bins, which produced the lower right panel of Fig. 3.7, to see no noticeable features. This average and the preceding one suggest that there is no sub-pulse drifting within either mode of $\mathrm{J} 1752+2359$. 


\subsection{Conclusion}

In summary, by examining the pulse energy distribution and positive number statistics, we have been able to reveal a low energy normal mode with in J1752+2359 in archival data set taken with the Arecibo radio telescope.

It was found that the pulse energy distribution could best be described by a combination of a log-normal and normal distribution. The log-normal distribution seemed to consist primarily of the bursting events and large single pulses. The normal distribution had a small offset that was statistically significant. This offset hinted that an underlying normal mode. By averaging the pulses with the pulse energies that made the majority of the Gaussian distribution, a pulse profile became clear with the same energy as the original offset. To ensure that this was not due to outlying events, the distribution that generated the mean peak flux was examined and was shown to be a Gaussian distribution with a deviation similar to the noise. This distribution is consistent with a constantly emitting normal mode that has been affected by system noise.

To further ensure that this profile was not due to outlying events, we tested the number of positive flux values and their statistics. This testing supports a regular occurring normal mode by tracking the signal-to-noise through different methods. By optimizing these statistics, we are able to approximate a profile and improve the signal-to-noise of the original profile by over ten percent. Each method demonstrated an increase in the probability of receiving a positive value that corresponds with the normal mean profile. This further supports that these pulses are reoccurring as 
expected for a constantly emitting normal mode that has been affected by system noise.

This discovery of this normal mode in the bursting J1752+2359 is consistent with what has been found in other bursting pulsars thus far. To see if J1752+2359 was similar to other bursting pulsars in other ways, we searched for sub-pulse drifting. Through a novel way of utilizing each event to improve the fluctuation spectrum for each mode, we were able to show that there is no prominent frequency in either modes' fluctuation spectrum. This implies that there is no sub-pulse drifting present in this pulsar, which is consistent with the bursting pulsar B0611+26 discussed in Chapter 2 .

With longer observations, we hope to increase the signal-to-noise on the average normal profile to determine if there are phase shifts between the modes as observed in the other bursting pulsars. Longer observations will also help determine whether the bursting events are random or have a deterministic behavior by improving the burst interval statistics. 


\section{Chapter 4}

\section{Dynamical Study of Pulsar Spin-down Rates ${ }^{1}$}

\subsection{Linear Analysis}

We wish to search for chaotic and non-linear behavior in the spin-down rate presented in fig. 2 of Lyne et al. (2010). There they isolated a subset of 17 pulsars with prominent variations in their frequency derivatives. These time series are ideal for non-linear studies because they directly relate to the dynamics of a pulsar. Before non-linear analysis can be done, we need to compensate for some their limitations.

\subsubsection{Mind the gap}

When dealing with long time-scales, such as those encountered in astronomy, it is not always feasible to record data at regular intervals. This produces a time series that sporadically samples a continuous phenomenon. When the time spacing between two points in the series is relatively small, little information is lost about the continuum inside that region. If the spacing is large, more information is lost, which can cause a significant change in the structure of the data.

To avoid such changes, we would like to analyze the largest section in the time series that best samples the phenomenon. We start by finding the statistical mode of the spacing, which gives us the step size that is the closest to being evenly sampled.

\footnotetext{
${ }^{1}$ Much of the work presented in this chapter has been published in Seymour \& Lorimer (2013).
} 


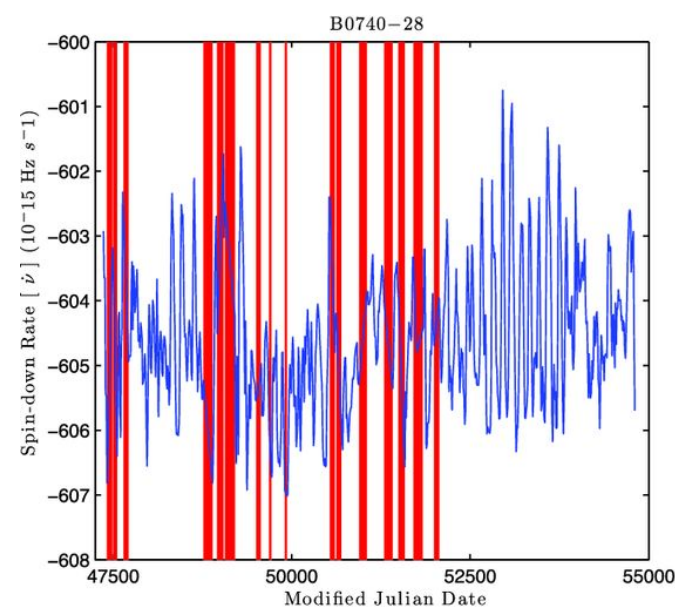

(a)

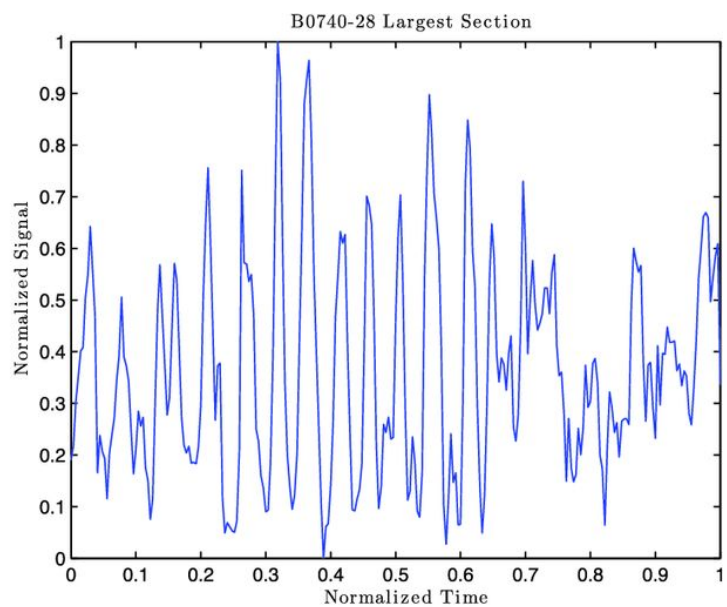

(b)

Figure 4.1: (a) The time series of measured spin-down rates computed from recorded data for B0740-28. The vertical red bars highlight the gaps that are larger than three times the mode spacing. (b) The largest section for B0740-28, seen in the last section in Fig. 4.1a, after being normalized on both axes.

We then compare this with the spacings in the series. If a gap is larger than three times the mode spacing, we assume that this region has significant information loss.

The time series is now broken into several sections that are separated by these large gaps, an example of which can be seen in Fig. 4.1a. We extract the longest section of data which is then normalized on both axes for more efficient computing, as seen in Fig. 4.1b.

\subsubsection{Turning point analysis}

We want to make sure that the new time series is depicting a real phenomenon and is not solely a consequence of noise. If the time series samples a continuous function well, then we should expect a smooth curve with a large number of data points between maxima and minima, or what are called turning points. If the time series samples a random distribution, then we should expect several rapid fluctuations 


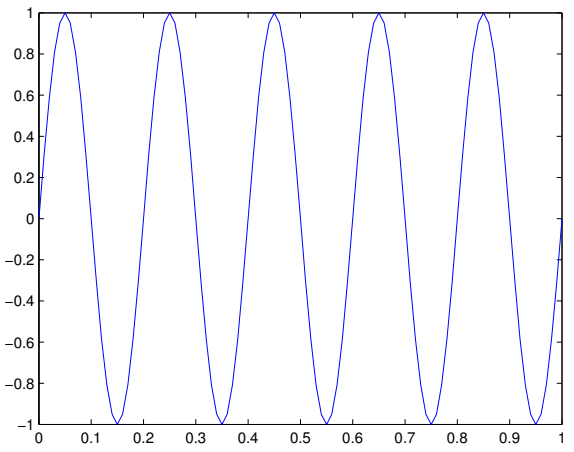

(a)

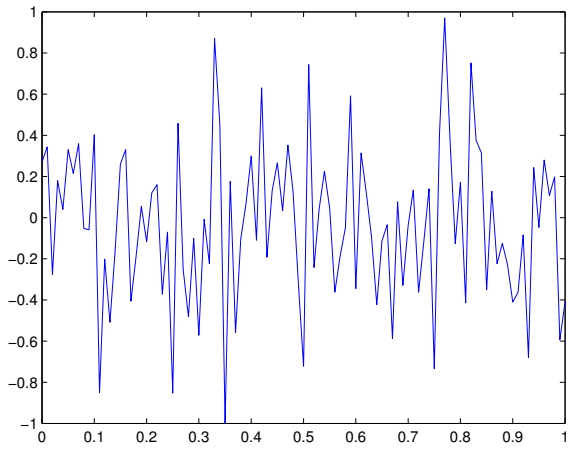

(b)

Figure 4.2: (a) Sine function: an example of a continuous function. (b) Random time series with the same number of data points as the sine wave. Here both axes are in arbitrary units.

which will cause a large number of turning points. We can see an example of this in Fig. 4.2, where the well sampled continuous sine wave has very few turning points, while the random series, with the same number of data points, has considerably more.

In order to compare a series to noise, we need to have some expectation of the number of turning points that should be in a random series. It takes three consecutive data points to create a turning point. When randomly sampling, it is virtually impossible to get two values that are exactly the same. Therefore, the values of the three data points will have the relationship, $u_{1}<u_{2}<u_{3}$ (Kendall \& Stuart, 1966). These values can be rearranged in six different combinations, as seen in Fig. 4.3, where we find that four will produce a turning point (Kendall \& Stuart, 1966). This means that any enclosed data point will have a $2 / 3$ probability of being a turning point. The first and last data points in a series of size $n$ are the only unenclosed points. Thus, there are $(n-2)$ possible locations where a turning 
point can occur. This leads to an expectation value

$$
\mu_{T}=\frac{2}{3}(n-2)
$$

and, as described in Appendix B, a variance

$$
\sigma_{T}^{2}=\frac{16 n-29}{90}
$$

If the number of turning points is

greater than the expected value, the se-

ries is fluctuating more rapidly than ex-

pected for a random time series (Brock-

well \& Davis, 1996). On the other hand,

a value less than the expected value indi-

cates an increase in the number of data

points between each turning point.

To be confident that the signal is

$$
\begin{aligned}
& u_{1}, u_{2}, u_{3} \\
& u_{1}, u_{3}, u_{2} \\
& u_{2}, u_{1}, u_{3} \\
& u_{2}, u_{3}, u_{1} \\
& u_{3}, u_{1}, u_{2} \\
& u_{3}, u_{2}, u_{1}
\end{aligned}
$$

Figure 4.3: Illustrations showing the combinations of data points for which $u_{1}<$ $u_{2}<u_{3}$. See text for details.

not a result of random fluctuations, we

will only keep a series that has a total number of turning points that are five standard deviations less than the expectation value of a series of the same size. If a time series has a total number of turning points greater than this value, it is indistinguishable from noise and will not be used. This does not necessarily imply that there is 


\begin{tabular}{|c|c|c|c|c|c|}
\hline Pulsars & $T$ & $n$ & $\mu_{T}$ & $\sigma_{T}$ & $\begin{array}{c}\# \text { of } \sigma_{T} \mathrm{~s} \text { away } \\
\mathrm{T} \text { is from } \mu_{T}\end{array}$ \\
\hline B1828-11 & 56 & 259 & 171.33 & 6.76 & -17.06 \\
\hline B0740-28 & 105 & 270 & 178.67 & 6.90 & -10.67 \\
\hline B1826-17 & 34 & 123 & 80.67 & 4.64 & -10.05 \\
\hline B1642-03 & 53 & 154 & 101.33 & 5.20 & -9.29 \\
\hline B1540-06 & 19 & 74 & 48.00 & 3.58 & -8.10 \\
\hline B2148+63 & 25 & 81 & 52.67 & 3.75 & -7.37 \\
\hline B0919+06 & 31 & 90 & 58.67 & 3.96 & -6.99 \\
\hline B1714-34 & 7 & 39 & 24.67 & 2.57 & -6.87 \\
\hline B1818-04 & 23 & 73 & 47.33 & 3.56 & -6.84 \\
\hline $\mathrm{J} 2043+2740$ & 12 & 44 & 28.00 & 2.74 & -5.84 \\
\hline B1903+07 & 30 & 79 & 51.33 & 3.70 & -5.76 \\
\hline B0950+08 & 29 & 76 & 49.33 & 3.63 & -5.60 \\
\hline $\mathrm{B} 1907+00$ & 32 & 80 & 52.00 & 3.73 & -5.36 \\
\hline$\overline{\mathrm{B}} \overline{2} \overline{0} \overline{5} \overline{5}+\overline{3} \overline{6}$ & $\overline{1} \overline{4}$ & $\overline{43}$ & $\overline{2} \overline{7} . \overline{3} \overline{3}$ & $\overline{2} . \overline{1}$ & $-\overline{-4.93}-$ \\
\hline $\mathrm{B} 1929+20$ & 11 & 33 & 20.67 & 2.35 & -4.11 \\
\hline B1822-09 & 56 & 108 & 70.67 & 4.34 & -3.38 \\
\hline B1839+09 & 13 & 31 & 19.33 & 2.28 & -2.78 \\
\hline
\end{tabular}

Table 4.1: The 17 pulsars listed in order of their distance away from $\mu_{T}$. Here $T$ is the total number of turning points in the extracted series. $n$ is the total number of data points in that series. $\mu_{T}$ is the expected number of turning point for a random series of size $n$, and $\sigma_{T}$ is the standard deviation of the expected value. The dashed line marks the five standard deviation threshold. The negative values indicate that these series are 'smoother' than an expected random series with the same number of data points.

not a phenomenon present. Rather that the data may not sufficiently sample the phenomenon.

In Table 4.1 we have listed the values for the time series that were extracted from the corresponding pulsar. Unfortunately, the last four pulsars failed to pass the detection threshold, and are excluded from any further analysis. 


\subsubsection{Cubic spline}

Most signal processing techniques assume that a time series is evenly sampled, and when the series is spaced randomly these algorithms severely increase in complexity. Therefore we would like to form an evenly sampled series that has the same structure as our own. Since we have selected a section that is nearly evenly spaced and we are reasonably confident that a signal is resolved, we can use a cubic spline interpolation to approximate the structure in between data points. After the data have been splined, we then resample with a step size equal to the statistical mode of the original spacing. This is done with the intent of limiting a significant increase in the time resolution.

\subsubsection{Fourier transform}

Having more data points will be beneficial in upcoming analyses, but caution must be taken to prevent introducing structures or signals that are not present in the series. If we take the Fourier transform of the data, we will have an amplitude and a phase at each frequency below the Nyquist frequency. It is important to emphasize that we receive both amplitude and phase only when the data are evenly sampled. These can in return be used to construct an approximate time function for the series. This function can then be sampled at any rate without adding any significant nonpre-existing frequencies as described below. 


\subsubsection{Noise reduction}

Even though the turning point analysis convinced us that our series is not governed by noise, this does not mean to say that there is no noise in the data. Before generating the new time series, we take the opportunity at this point to perform noise reduction.

The simplest method is a low pass filter, but a decision needs to be made as to where to place the cut-off frequency. One can view our time series as the addition of two series: a signal series plus a noise series of equal size. From our turning point analysis, we know the expected number of total turning points for the noise series, and on average we should expect the minima and maxima to be equally spaced. Therefore, on average the noise should contain a frequency

$$
f_{\text {noise }}=\frac{\mu_{T}}{2 \Delta t_{\text {total }}}
$$

were $\Delta t_{\text {total }}$ is the total time recorded. We then extend the frequency to a value corresponding to two more standard deviations below the expected number. Our data have been normalized in time, so that they only cover a single time unit. This leads us to set an angular cut-off frequency

$$
\omega_{\max }=\pi\left(\mu_{T}-2 \sigma_{T}\right) .
$$

We are now able to approximate a time function by doing an inverse Fourier transform up to the cut-off frequency. Since the Fourier series is a sum of periodic 


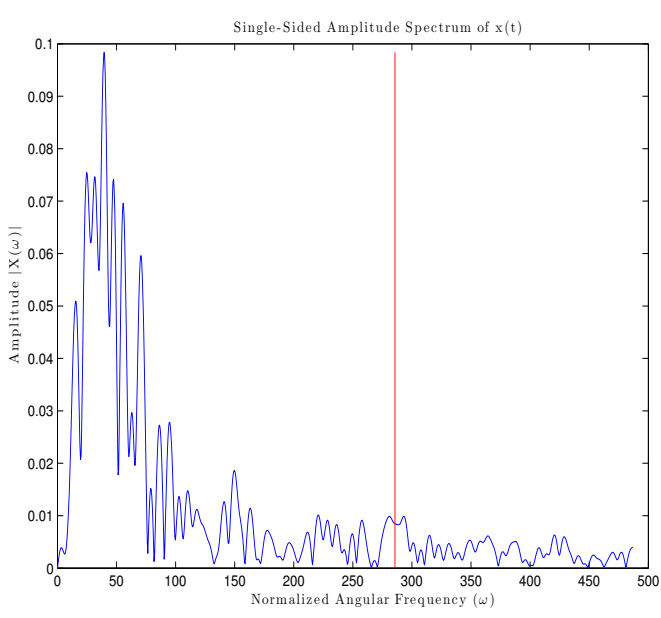

(a)

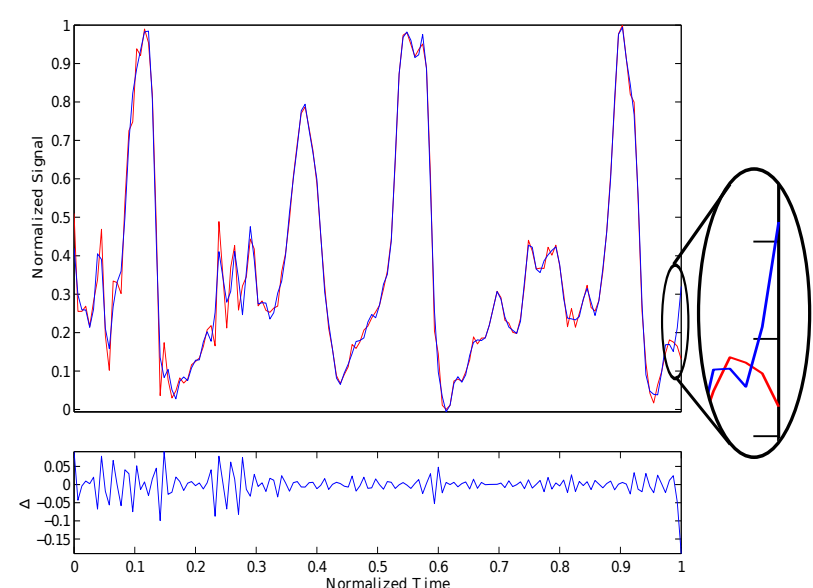

(b)

Figure 4.4: (a) The Fourier transform amplitudes of B1642-03. The vertical red line marks the cut-off frequency. (b) Top: The pulsar data (red) overlaid with Fourier time function with the same cadence (blue). Bottom: The difference of the two curves. Note that at the end of the series, the structure varies considerably among them.

functions, the new time series will, by definition, repeat back upon itself. This can cause noticeable errors towards the beginning and end of the time series, as seen in Fig. 4.4, and to avoid this we will not sample within the first and last five percent of the time recorded. We then generate the time series by evenly evaluating the time function until we have 5,550 data points. This leaves us with approximately 5,000 data points within the desired range.

\subsection{Non-linear Analysis}

For the non-linear analysis we use a combination of the TISEAN software package presented by Hegger et al. (1999) and our programs written in the MATLAB programing language. We intend to have the MATLAB programs available to the reader on the MathWorks file exchange website to help aid in the understanding of the algorithms. 


\subsubsection{Attractor reconstruction}

With only one observable, it seems that we are unable to reproduce an attractor, but surprisingly the dynamic series contains all the information needed for its reconstruction.

\subsubsection{Method of delays}

We can utilize embedding theorems developed by Takens (1981) and by Sauer et al. (1991) to reconstruct the attractor. These theorems state that a series of scalar measurements of a dynamical system can provide an one-to-one image of a vector set, the strange attractor, through time delay embedding (Hegger et al., 1999). Each element in the vector is the scalar measurement $S(t)$ at a different time as follows

$$
\mathbf{x}(t)=[S(t), S(t+\tau), S(t+2 \tau), \ldots, S(t+(m-1) \tau)]
$$

The number $m$ of elements in the vector is said to be the embedding dimension (Hegger et al., 1999), while $\tau$ is a time delay.

\subsubsection{Time delay}

It soon becomes evident that picking the proper time delay is crucial. If the time delay is too small, then each element will be very close in value, forming a tight cluster. If the delay is too large, then the elements are unrelated and the attractor information is lost. If we were simulating a solution to non-linear equations, we would 


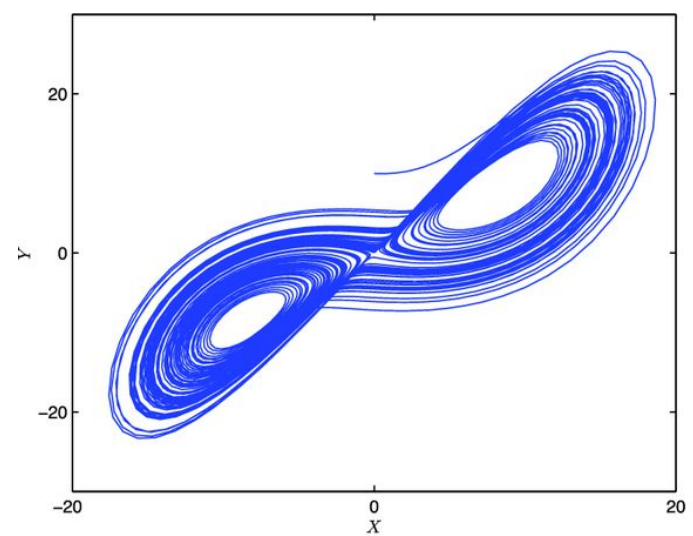

(a)

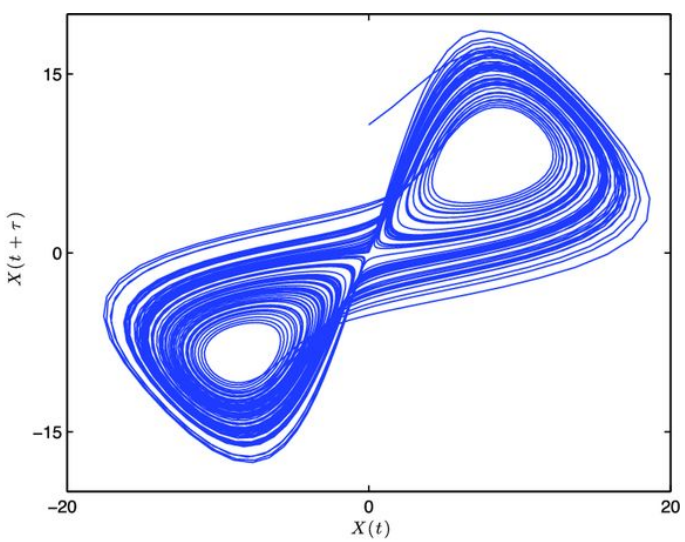

(b)

Figure 4.5: (a) The Lorenz attractor XY plane under the same condition as Fig. 1.13a. (b) The reconstructed attractor from only the $\mathrm{X}$ values with $\tau=0.1345$, which was estimated using the autocorrelation coefficients.

have to find a time-scale where linear effects are dominant and to make sure that our step size was within this range. We would like to do the same thing but for the time delay, because the orthogonal axes will differ by a dynamically linear trigonometric function.

There are a wide range of algorithms that are used to find this appropriate time delay, but the simplest ones fail to account for non-linear effects (Hegger et al., 1999). The algorithms that do account for these effects are not very intuitive. We chose to use adaptations of turbulent flow techniques which can be seen in Mathieu \& Scott (2000).

One way of estimating this linear range is to use the autocorrelation coefficients. We are concerned with the structure of the fluctuations and would like to have a zero average signal $s(t)$. This is readily done by subtracting the time average of the series from each scalar measurement. We then generate the autocorrelation coefficients for 
this new signal, defined as

$$
\rho(\Delta t)=\frac{<s(t) s(t+\Delta t)>}{<s(t)^{2}>}
$$

This function will generate a curve that will start at one and then taper to zero, seen in Fig. 4.6.

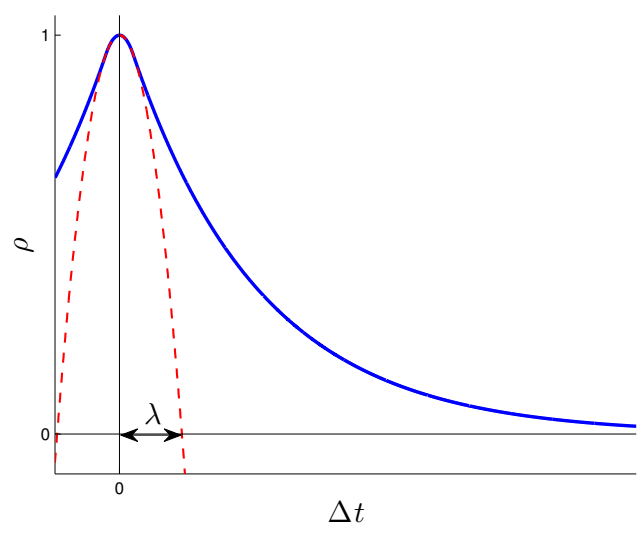

Figure 4.6: An ideal example of autocorrelation coefficients. The blue curve is the autocorrelation coefficients. The red dashed curve is a parabolic fit to the autocorrelation on small time-scales. $\lambda$ is the time-scale estimate where linear relation- set the time delay to half of this value. ships are believed to be dominant.
Based on the second order Taylor series expansion of $\rho(\Delta t)$, we can estimate the linear region by fitting a parabola to the small $\Delta t$ region of $\rho(\Delta t)$. The positive root of this parabola is our estimate, $\lambda$, for a linear time-scale. This means that time-scales up to $\lambda$ should be dominated by linear effects, but to ensure that we are well into this region, we We can see how well this estimate works in Fig. 4.5, where the topology of the attractor has been conserved.

When these techniques are applied to the pulsar time series, a similar topology appears among the best-sampled pulsars, which is seen in Fig. 4.7. At first glance, they seem to mimic different paths along a similar attractor, perhaps demonstrating 
a route to chaos. One could interpret B1540-06 as periodic behavior, B1828-112 as period two behavior, and B1826-17 and B1642-03 as chaotic. To be sure that this is truly the case, we need to measure the dimension of each topology.

\subsubsection{Correlation dimension}

There are several different algorithms that are used to measure the fractal dimension, but nearly all depend on a power-law relationship. The most widely used is the correlation dimension, first introduced by Grassberger \& Procaccia (1983).

It is calculated by creating a test sphere of radius $R$ centered on a data point located at $\mathbf{x}$. The number of data points inside the sphere is then counted, $N_{x}(R)$. The radius is slowly increased. As it increases, the number of data points in the sphere grows as a power-law (Strogatz, 1994)

$$
\begin{gathered}
N_{x}(R) \propto R^{d} \\
d=\frac{d\left(\ln N_{x}\right)}{d(\ln R)} .
\end{gathered}
$$

Here $d$ is referred to as the pointwise dimension at $\mathbf{x}$. Fig. 4.8 shows this relationship with familiar geometries.

Due to fluctuations in the sampling density, the pointwise dimension can vary depending on where $\mathbf{x}$ is located. In order to produce a more self consistent measurement, an average of $N_{x}(R)$ is taken over all data points. This average is known as

\footnotetext{
${ }^{2} \mathrm{~A}$ linear trend was removed in order to make the time series stationary, behavior because we are solely interested in the fluctuations. When B1828-11 is referenced in the rest of the paper, it is implied that this linear trend has been removed.
} 


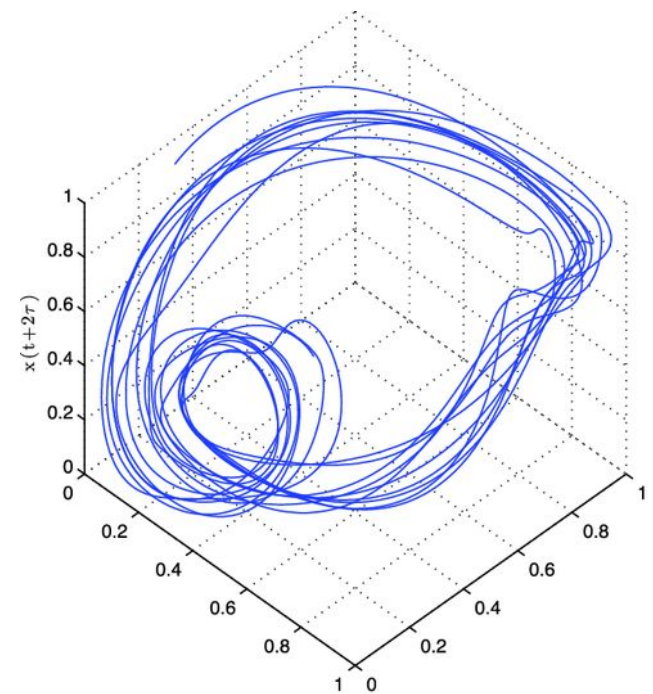

$\mathrm{x}(\mathrm{t}+\tau)$ $x(t)$

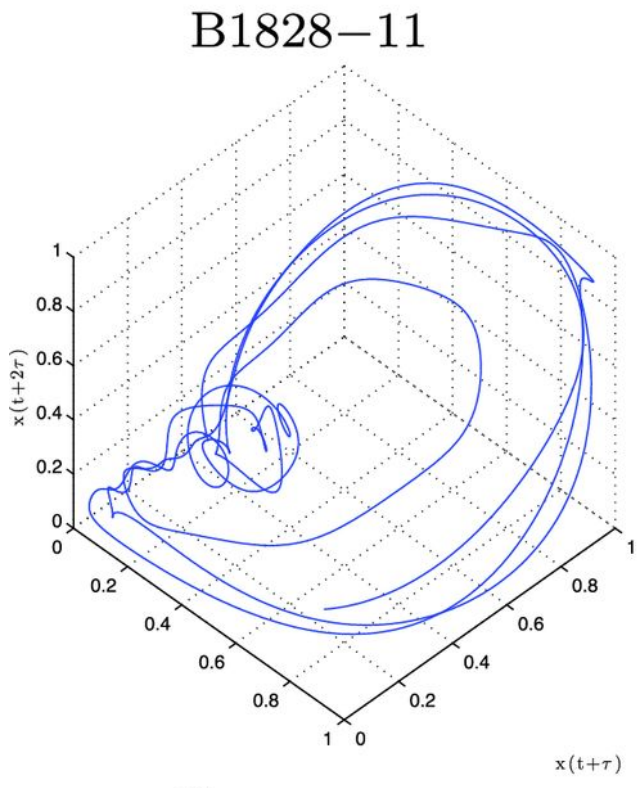

$x(t)$

B1642-03

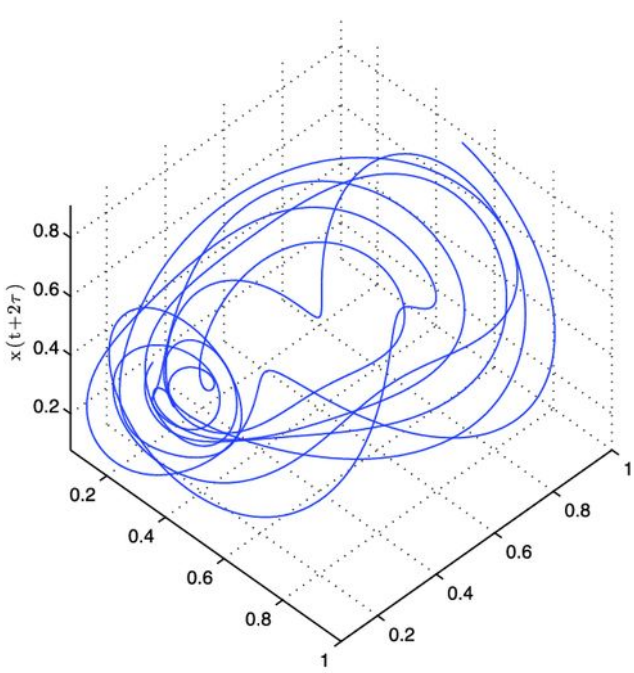

$x(t)$

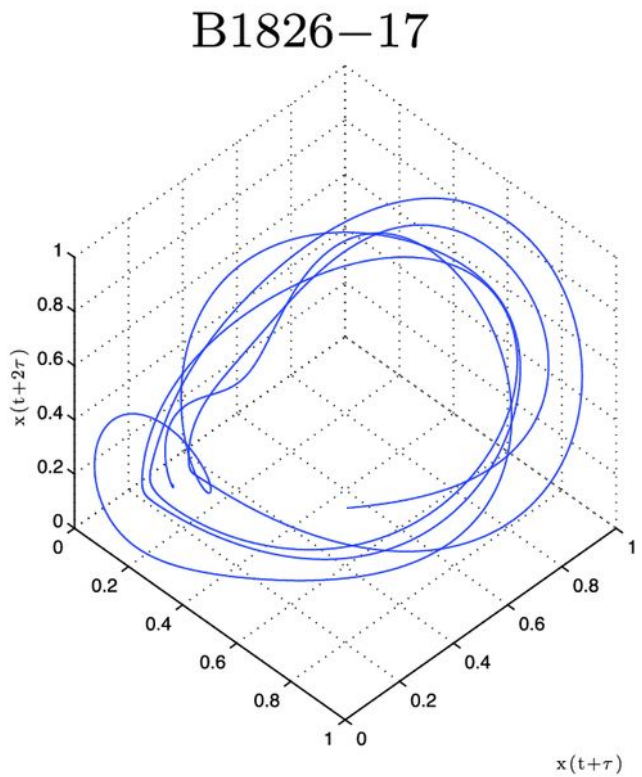

$x(t)$

B1540-06

Figure 4.7: The topology of the pulsar time series after being embedded in three dimensional space with coordinates of $[X(t), X(t+\tau), X(t+2 \tau)]$. 


\begin{tabular}{c|c|c} 
Geometry & $N_{x}$ & $\frac{d \ln N_{x}}{d \ln R}$ \\
\hline & $2 R$ & 1 \\
& $\pi R^{2}$ & 2 \\
& & \\
& & \\
& & \\
& & \\
& &
\end{tabular}

Figure 4.8: $N_{x}$ is the number of data point within the radius R. $N_{x}$ will be proportional to the equations for each given situation. We can see that $\frac{d \ln N_{x}}{d \ln R}$ will produce the correct dimension for each of these situations.

the correlation sum, $C(R)$, and is often written as

$$
C(R)=\frac{2}{N(N-1)} \sum_{i=1}^{N} \sum_{j=i+1}^{N} H\left(R-\left\|\mathbf{x}_{\mathbf{i}}-\mathbf{x}_{\mathbf{j}}\right\|\right)
$$

where $H$ is the Heaviside function, $H(x)=0$ if $x \leq 0$ and $H(x)=1$ if $x>0$. $N$ is the total number of locations. \|\| is the magnitude of the vector such that $\|\mathbf{v}\|=(\mathbf{v} \cdot \mathbf{v})^{\mathbf{1} / \mathbf{2}}$

This correlation sum will have the exact same power relation as $N_{x}(R)$. The exponent for $C(R)$ is then properly named the correlation dimension. This is defined as

$$
D=\lim _{N \rightarrow \infty} \lim _{R \rightarrow 0} \frac{d(\ln C)}{d(\ln R)}
$$

We seldom have the luxury of infinite sample size with infinitely small resolution, and therefore different behaviors occur in the correlation sum.

To estimate $D$, one would plot $\ln C$ versus $\ln R$. If the power relationship held true for all $R$ we would see a straight line with a slope of $D$. However, due to the 
finite size of the attractor, all points could lie within a large enough $R$. We can see in Equation 4.8 that this would cause $C(R)$ to converge towards a value of one. At low enough $R$ we will reach a resolution limit, where only the center data point is inside the test sphere. This causes $C(R)$ to converge to zero. Therefore the power-law will only hold true over an intermediate scaling region.

We could safely avoid this resolution limit if 10 points were in each of our test spheres. This would correspond to $C(R)=\frac{10}{N}$, which we use as our lower limit of the scaling region. To set an upper limit, we say that if $10 \%$ of all points are in each test sphere, we would begin to approach the scale of the attractor. Therefore, we set our upper bound at $C(R)=0.10$ to close off a rough scaling region.

With this definition of dimension, one can look how there is a transition between integers. We can increase the number of data points of a line within $R$ by simply folding that line inside a test radius, perhaps like $S_{1}$ in Fig. 1.5.1. In order to have a power-law relationship across all sizes of $R$, we need to have a similar fold on all scales, known as self similarity. If we were to steepen the angles of each fold, this would increase the number contained and also increase the dimension. We can do this until the folds are directly on top of one another to 'color in' a two dimensional surface. We could then repeat the process by folding the two dimensional surfaces to transition to three dimensions. Folding like this is regarded as the underlying reason why strange attractors have their fractal dimensions and is explored in depth by Smale (1967) and Grassberger \& Procaccia (1983). 


\subsubsection{Theiler window}

If the attractor was randomly sampled we would be ready to measure the dimension, but the solutions are one dimensional paths on a multi-dimensional surface. Therefore, we have two competing dimension values which will corrupt our measurement. What we wish to do is to isolate only the attractor dimension, the geometric correlations, and remove the path relationships, the temporal correlations.

As complicated as this sounds, Theiler (1986) introduced a rather simple remedy. We exclude the points within a time window, $w \times \Delta t_{s}$, around the center of the test radius. Here $\Delta t_{s}$ is the step size of the time series, and $w$ is an integer value. This is known as the Theiler window and changes the correlation sum in the following way

$$
C(R)=\frac{2}{(N-w)(N-w-1)} \sum_{i=1}^{N} \sum_{j=i+w+1}^{N} H\left(R-\left\|\mathbf{x}_{\mathbf{i}}-\mathbf{x}_{\mathbf{j}}\right\|\right) .
$$

We can see that picking the appropriate $w$ is nontrivial. If we pick a window that is too small we fail to remove the temporal correlations, and if the window is too large it would significantly reduce the accuracy of the geometric measurement.

\subsubsection{Space time separations}

In order to estimate a safe value for the Theiler window, Provenzale et al. (1992) introduced the space time separation plot. It shows the relationship between the spatial and temporal separations, by forming a contour map of the percentage of locations within a distance, for a given time separation. 


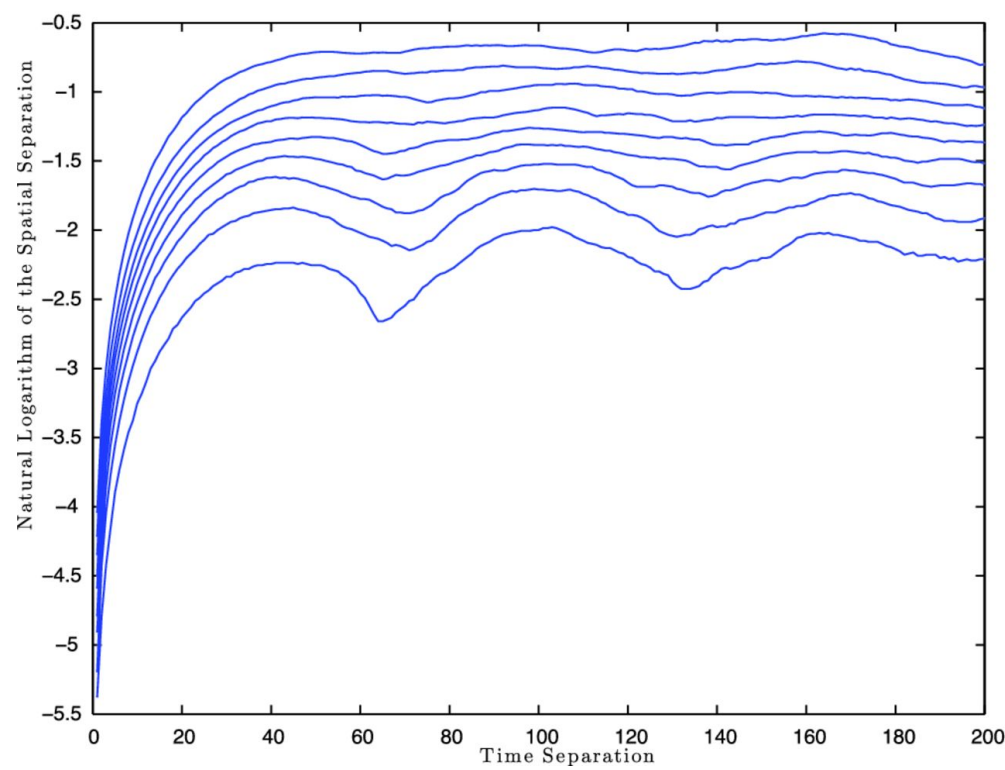

Figure 4.9: Space time separation plot, generated using the TISEAN package, for B1642-03 with $m=3$. The contours indicate the percentage of locations within a distance at a given time separation. The different curves correspond to $10 \%$ increases starting with the lowest curve at $10 \%$ to the top curve at $90 \%$.

An example of this is seen in Fig. 4.9; here we used the stp program in the TISEAN package. We can see that the locations with small time separations are close to one another. As the time separations increase, so do their spatial separations, until they reach some asymptotic behavior. The substantial fluctuations at larger times are contributed by the cycle period of the attractor (Kantz \& Schreiber, 2004). Temporal correlations are present until the contour curves saturate (Kantz \& Schreiber, 2004). In Fig. 4.9 this transition seems to occur around a time separation of 40 steps. This is a similar quantity that appears in all of our data sets, but to be safe we choose a more conservative value of $w=100$ steps for the rest of our analysis. 


\subsubsection{Embedding to higher dimensions}

We are ready to measure the dimensions of the topologies that were created in Section 4.2.1. If these topologies are true geometric shapes, their dimensions will not change when placed in a higher dimensional space. This means that as long as our embedding dimension, $m$, is greater than the attractor dimension, our correlation dimension measurement will be constant. On the other hand, if we were to embed a random distribution, it would be able to occupy the entire space and would have a correlation dimension equal to the embedding dimension.

Because of this behavior, we can embed our time series to higher and higher dimensions to see if it will plateau to a constant. We cannot do this forever because the number of location vectors that could be formed drops off as

$$
N=n_{s}-\tau^{*}(m-1),
$$

where $n_{s}$ is the total number of scalar values in the original time series and $\tau^{*}$ is the number of time steps, $\Delta t_{s}$, corresponding with the time delay, $\tau$. This drop off causes a slight reduction in our statistical accuracy as we continue to higher dimensions. Therefore, we limit our embedding dimensions to between 2 and 10 .

Though we have picked a rough scaling region in Section 4.2.1.3, $C \approx \frac{10}{n_{s}}$ to 0.10, this does not mean that the true scaling region extends through this entire range. Therefore, we sweep the region with a window size from a third of the total logarithmic range to the entire range, searching for the minimum deviation in the 


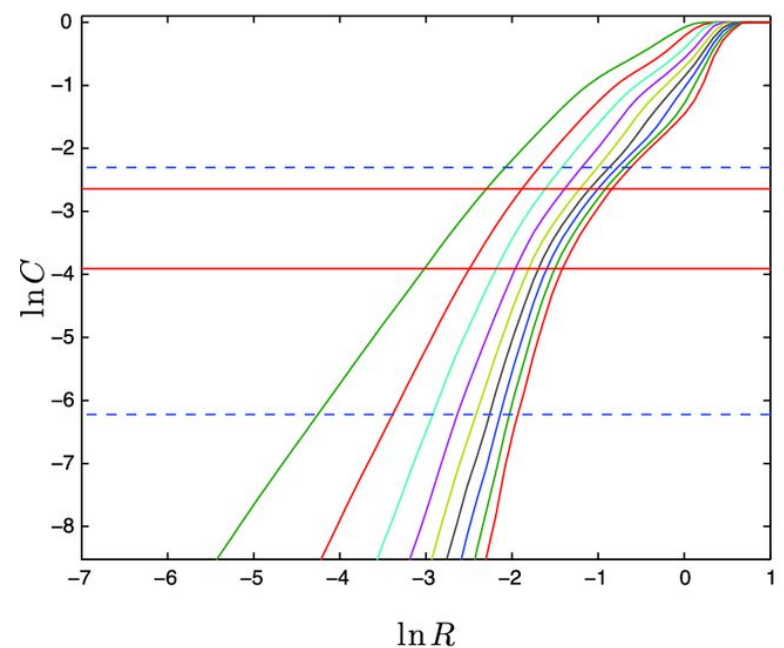

(a)

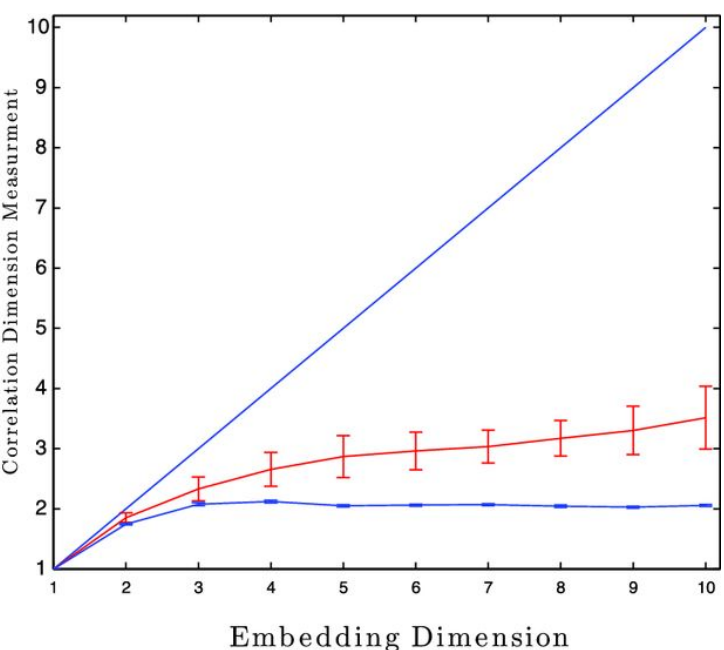

(b)

Figure 4.10: (a) $\ln C$ versus $\ln R$ for B1828-11. The dashed horizontal blue lines mark the rough scaling region, $C=\frac{10}{n_{s}}$ to 0.10 . The solid horizontal red lines mark the flattest scaling region that is larger than a third of the rough scaling region. (b) The flat blue line is the correlation dimension measurements, within the scaling in (a), versus embedding dimension. The line at $45^{\circ}$ is what a purely random time series would be in that given $m$. The sloped red line is the mean and standard deviation of 10 surrogate data sets (see text).

correlation dimension measurements over the embedding dimensions. This ensures that we are picking the 'flattest' possible section over a significant scale.

When this technique is applied to the four pulsars from Fig. 4.7, two pulsars plateau very nicely. B1828-11, seen in Fig. 4.10, averages to a correlation dimension of $2.06 \pm 0.03^{3}$, and B1540-06 averages to $2.50 \pm 0.09$, for embedding dimensions greater than 3. The other two pulsars fail to converge to a constant. We attribute this non-convergence to the sparse coverage, where each pass around the attractor is too far apart to get a proper measurement.

\footnotetext{
${ }^{3}$ The error calculation is presented in appendix $\mathrm{C}$
} 


\subsubsection{Surrogate data}

We want to guarantee that any plateaus are due to geometric correlations and cannot be produced by random processes. Therefore, we would like to test several different time series with the similar mean, variance, and autocorrelation function as the original data set. These data sets are known as surrogate data.

The idea of surrogate data was first introduced in Theiler et al. (1992), but we use an improved version that was presented in Schreiber \& Schmitz (1996) for our surrogate data sets. They start by shuffling the order of the original time series, and then take the Fourier transform of this shuffled series. Keeping the phase angle of the shuffled set, they replace the amplitudes with the Fourier amplitudes of the original times series. Then they reverse the Fourier transform, and create the desired surrogate data set. Schreiber \& Schmitz (1996) iteratively do this until changes in the Fourier spectrum are reduced. Fortunately, this is all done in the program surrogates in the TISEAN package (Hegger et al., 1999), which we used for our analysis.

We form 10 surrogate data sets for each pulsar and run them through the same non-linear analysis as the post processed time series. We then find the average and standard deviation for the surrogates' correlation dimension for each embedding dimension to trace out the region where we should expect other surrogates to lie. We are confident that the original time series is not due to a random process if its correlation dimension measurements lie away from this region.

As seen in Fig. 4.10, B1828-11 is well outside the surrogate region, suggesting that it is a true dimension measurement. The measurements for B1540-06, seen in 


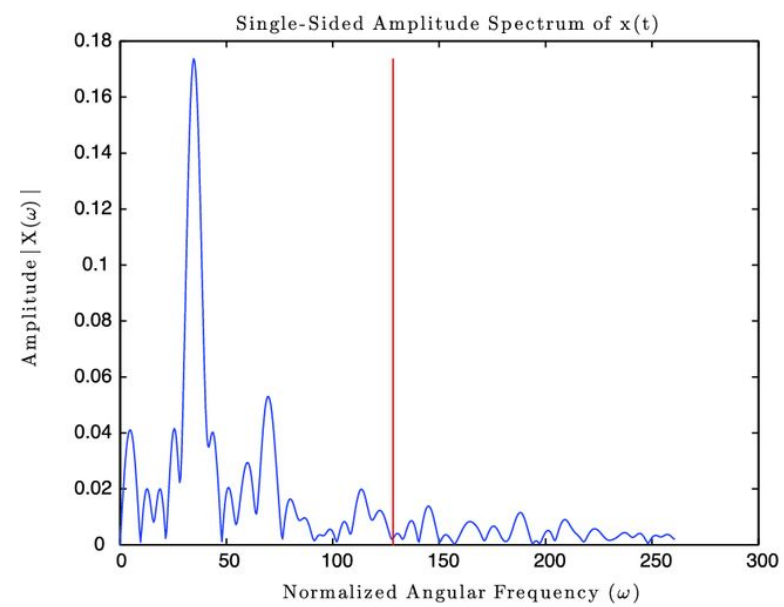

(a)

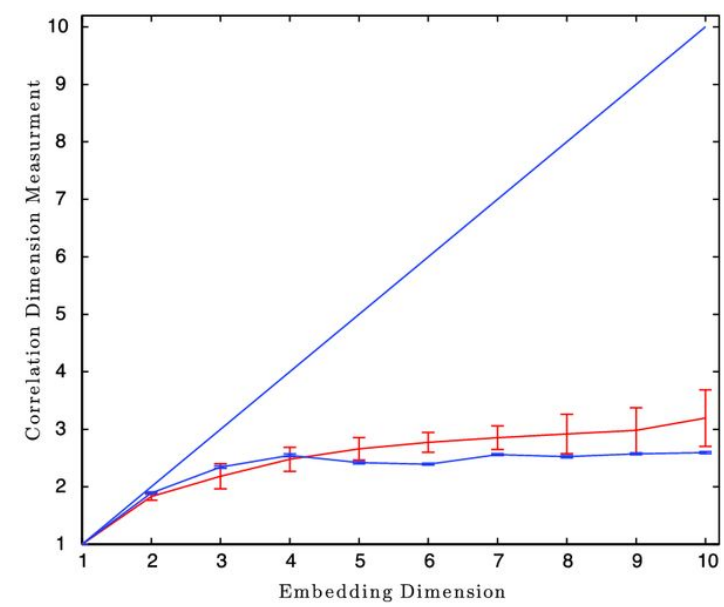

(b)

Figure 4.11: (a) The amplitudes of the Fourier series versus normalized frequency for B1540-06. The vertical red line marks where we set the cut off frequency. (b) The blue error bars show the correlation dimension measurement, and the red error bars show the surrogate data region for B1540-06.

Fig. 4.11, marginally miss this boundary, but because this pulsar is highly periodic, the Fourier spectrum is dominated by a single frequency. This restricts the complexity of the surrogate to where little change in dimension is expected. We will see more on this in Section 4.2.1.8.

\subsubsection{Correlation benchmark testing}

Though each part of the algorithm works independently, we want to ensure that it all works together correctly. Therefore we need to run through the process with a time series from known attractors under similar conditions to our original data, in order to see if we receive similar dimensions. For this analysis, we choose the Lorenz and Rössler attractors, whose dimensions have been documented as being $2.07 \pm 0.09$ and $1.99 \pm 0.07$ respectively (Sprott, 2003). 
We first generate the $x$ component time series for a corresponding attractor, making sure that our initial conditions are well on the attractor. Then, we extract a section from this time series out to the same number of turning points as used in the pulsar time series. This extracted section was then normalized on both axes, as we did in Section 4.1.1. We then resample the normalized time series by applying a cubic spline to the normalized times for the pulsar time series. This ensures that the new time series has the same time spacing and number of turning points as the pulsar time series. This new time series is then run through the algorithm to see if the proper dimension is achieved.

An example from the correlation dimension measurements are seen in Fig. 4.12, where we can see that due to the simplicity of the Rössler attractor in the frequency domain the surrogate data region only deviates by a small amount. We refer to this as a borderline detection. We also classify detections as being inside or outside based on their proximity to the surrogate region. Regardless, the correlation dimensions for both attractors plateau for all of the pulsar time series.

The results from all of the series are listed in Table 4.2. We can see that the algorithm does rather well considering we start with less than 300 data points. Although the algorithm is not refined enough to pinpoint the dimension, it is sufficient to determine the number of governing variables. If we round the dimension measurement to the nearest integer and then add one we will receive the proper number of variables for any series that is either outside or borderline to the surrogate region. 


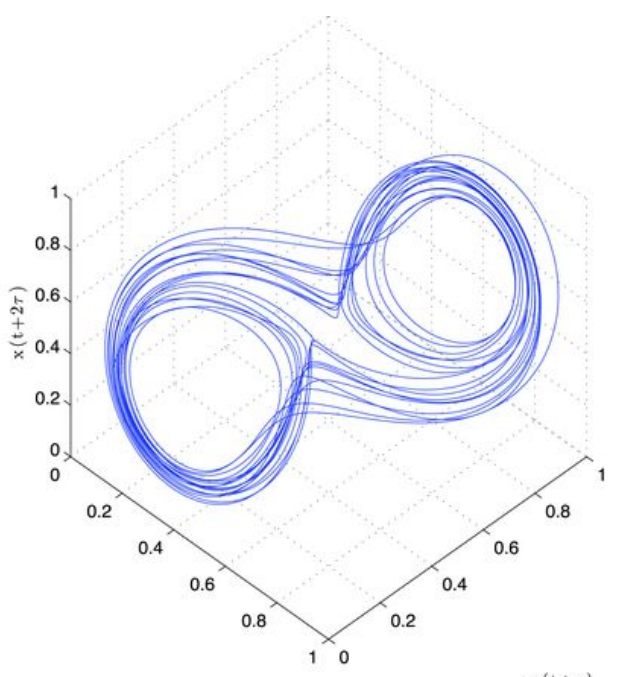

$x(t+\tau)$

$x(t)$
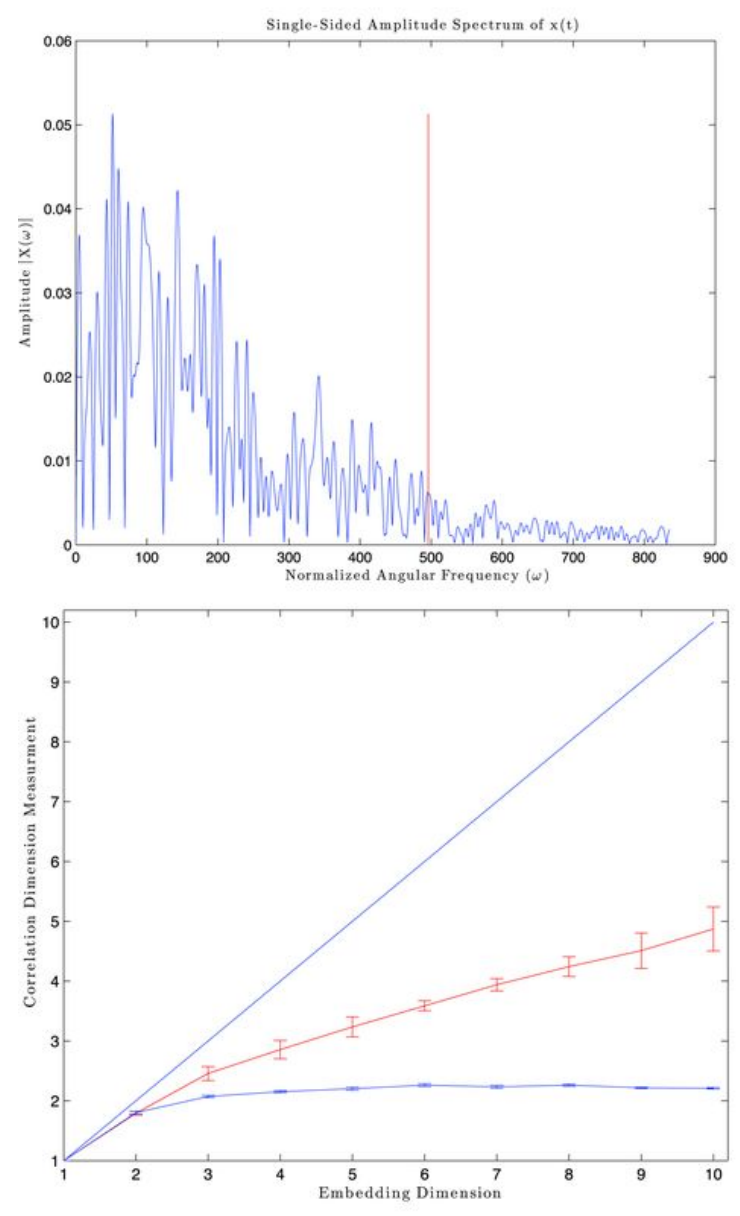

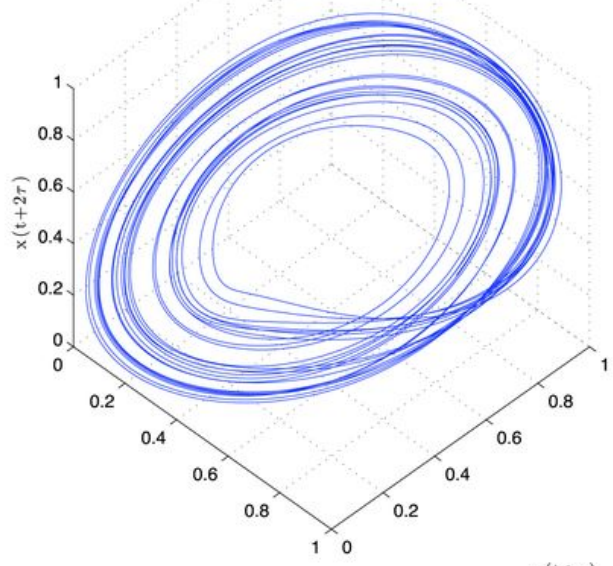

$x(t+\tau)$

$x(t)$
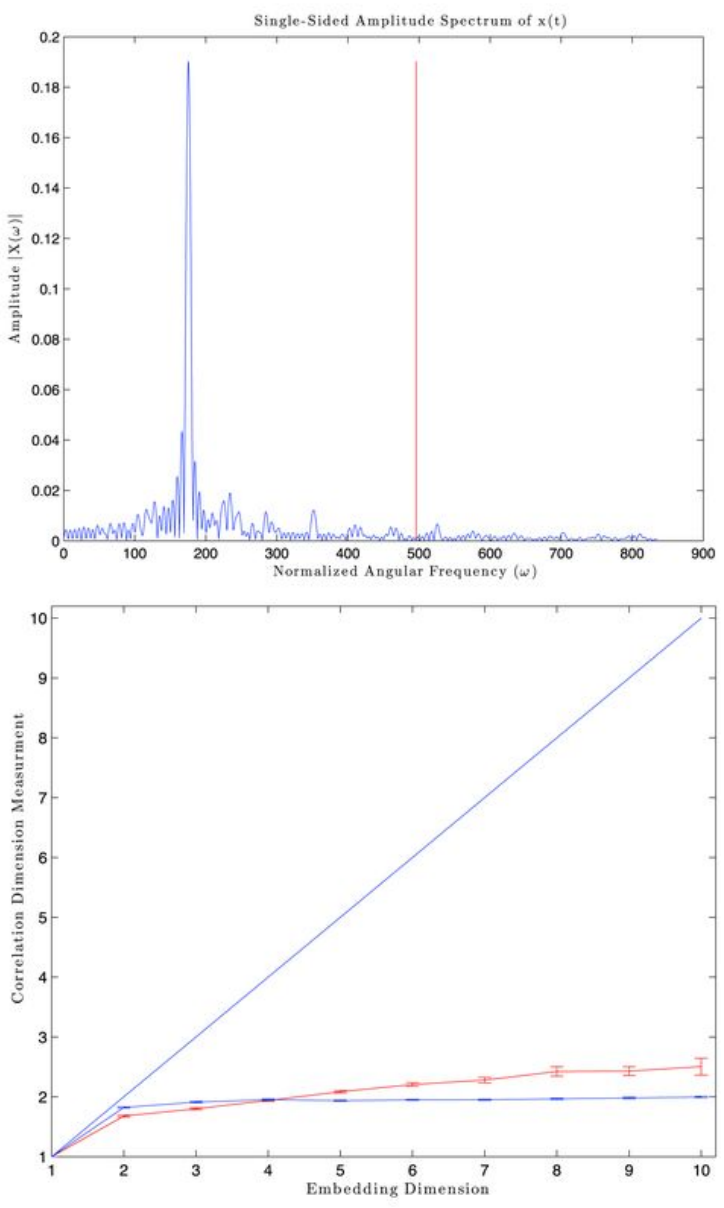

Figure 4.12: Benchmark results for B1828-11. Left column is the Lorenz attractor results. Right column is the Rössler attractor results. Top row is the reconstructed attractors in $[X(t), X(t+\tau), X(t+2 \tau)]$ space. Middle row is the Fourier transforms where the vertical red line marks the cut off frequency from Section 4.1.4.1. Bottom row is the correlation dimension measurements (blue error bars) with the surrogate region (red error bars). 


\begin{tabular}{lcccc} 
& \multicolumn{2}{c}{ Lorenz } & \multicolumn{2}{c}{ Rössler } \\
\hline & Correlation & Surrogate & Correlation & Surrogate \\
Pulsar & Dimension & Region & Dimension & Region \\
\hline B1828-11 & $2.20 \pm 0.06$ & O & $1.96 \pm 0.02$ & B \\
B0740-28 & $2.11 \pm 0.03$ & O & $2.16 \pm 0.08$ & B \\
B1826-17 & $2.00 \pm 0.10$ & O & $1.96 \pm 0.04$ & B \\
B1642-03 & $2.05 \pm 0.07$ & O & $2.00 \pm 0.10$ & B \\
B1540-06 & $1.90 \pm 0.20$ & O & $2.30 \pm 0.10$ & B \\
B2148+63 & $2.33 \pm 0.05$ & O & $2.13 \pm 0.02$ & B \\
B0919+06 & $1.90 \pm 0.10$ & O & $2.00 \pm 0.10$ & I \\
B1714-34 & $1.85 \pm 0.01$ & I & $2.87 \pm 0.07$ & I \\
B1818-04 & $1.70 \pm 0.05$ & O & $2.26 \pm 0.08$ & B \\
J2044+2740 & $1.83 \pm 0.03$ & O & $2.10 \pm 0.10$ & O \\
B1903+07 & $2.11 \pm 0.09$ & O & $2.00 \pm 0.20$ & I \\
B0950+08 & $1.68 \pm 0.09$ & O & $2.00 \pm 0.20$ & B \\
\hline Average & $2.00 \pm 0.20$ & & $2.00 \pm 0.10$ &
\end{tabular}

Table 4.2: The weighted average for the correlation dimension for $m>3$ for the corresponding pulsar and attractor. The surrogate region column marks whether the plateau line was either inside $(I)$, outside $(O)$, or borderline to $(B)$ the surrogate data region. The average row is the weighted average of the correlation dimension measurements that were either marked as $O$ or $B$.

\subsubsection{Measuring the butterfly effect}

Though our correlation dimension measurement can narrow the number of governing variables, it is not definitive enough on its own to say that our attractors are chaotic. Therefore, we need to search for other signs of chaos in our topologies to assure that they are indeed strange attractors.

\subsubsection{Lyapunov exponent}

As mentioned in Section 1.5, the butterfly effect is only present in chaotic systems. We should only see an exponential divergence in nearby locations over time if the system is chaotic. The exponent of this increase is a characteristic of the system 
and quantifies the strength of chaos (Kantz \& Schreiber, 2004). This is known as the Lyapunov exponent.

There are as many Lyapunov exponents as there are axes. Here we are only interested in measuring the maximum exponent because it gives us the most information about our system. We start by looking at the distance between two locations, $\delta_{0}=\left\|\mathbf{x}_{\mathbf{t}_{\mathbf{1}}}-\mathbf{x}_{\mathbf{t}_{\mathbf{2}}}\right\|$, then recording this separation over time, $\delta_{\Delta t}=\left\|\mathbf{x}_{\mathbf{t}_{\mathbf{1}}+\boldsymbol{\Delta} \mathbf{t}}-\mathbf{x}_{\mathbf{t}_{\mathbf{2}}+\boldsymbol{\Delta} \mathbf{t}}\right\|$. The Lyapunov exponent, $\lambda$, would be

$$
\delta_{\Delta t} \approx \delta_{0} e^{\lambda \Delta t}
$$

Since the separation between two points cannot be greater than the size of the attractor itself, Equation 4.12 will only hold true for $\Delta t$ values where $\delta_{\Delta t}$ is smaller than the attractor.

If $\lambda$ is positive, the system will be highly sensitive to initial conditions and therefore chaotic. If $\lambda$ is negative, the system would eventually converge to a single fixed point. If $\lambda$ is zero then this would represent a limit cycle where the path keeps repeating itself and is deemed to be marginally stable (Kantz \& Schreiber, 2004).

In actuality, the separations do not grow everywhere on the attractor and locally they can even shrink, and with contributions from experimental noise it is more robust to use the average to obtain the Lyanpunov exponent.

The algorithm for this averaging was introduced independently by Rosenstein et al. (1993) and Kantz (1994). One first picks a center point located at $\mathbf{x}_{\mathbf{t}_{\mathbf{0}}}$ and takes note of the data points within a radius of $\epsilon$. This is known as the neighborhood. For 
a fixed $\Delta t$, the mean $\delta_{\Delta t}$ is calculated across the whole neighborhood. The logarithm of this mean distance is then averaged over all points in the attractor. Therefore, one needs to compute

$$
S(\Delta t)=\frac{1}{N} \sum_{t_{0}=t_{1}}^{t_{N}} \ln \left(\frac{1}{\left|U\left(\mathbf{x}_{\mathbf{t}_{\mathbf{0}}}\right)\right|} \sum_{\mathbf{x}_{\mathbf{t}} \in U\left(\mathbf{x}_{\mathbf{t}_{\mathbf{0}}}\right)}\left\|\mathbf{x}_{\mathbf{t}_{\mathbf{0}}+\boldsymbol{\Delta} \mathbf{t}}-\mathbf{x}_{\mathbf{t}+\boldsymbol{\Delta} \mathbf{t}}\right\|\right),
$$

where $U\left(\mathbf{x}_{\mathbf{t}_{\mathbf{0}}}\right)$ is the neighborhood centered on $\mathbf{x}_{\mathbf{t}_{\mathbf{0}}}$. For our analysis, we choose a value of $\epsilon$ that is twice as large as our average distance to the nearest neighbor, while accommodating for the Theiler window. If the exponential relationship is present, we should see an overall linear behavior when $S(\Delta t)$ is plotted with respect to $\Delta t$. In order to conserve statistical accuracy we do not look beyond time-scales that are half the total time.

Similar to the correlation dimension, the Lyanpunov exponent is invariant to the number of embedding dimensions as long as $m>d$. Therefore we calculate $S(\Delta t)$ from $m=2$ to 10 to ensure that the slopes in the linear regions remain constant. When noise is present in deterministic systems, it causes a process similar to diffusion where $\delta_{\Delta t}$ expands proportionate to $\sqrt{\Delta t}$ on small scales (Kantz \& Schreiber, 2004). This causes $S(\Delta t)$ to have a $\frac{1}{2} \ln (\Delta t)$ behavior, which produces a steep increase over short time intervals.

When this is applied to B1828-11, seen in Fig. 4.13, three distinct regions appear. On small scales, we can see a convergence region due to a combination of a noise floor and non-normality (Hegger et al., 1999). Because of these effects, it takes the neighborhood a while to align in the direction of the largest Lyapunov exponent. 


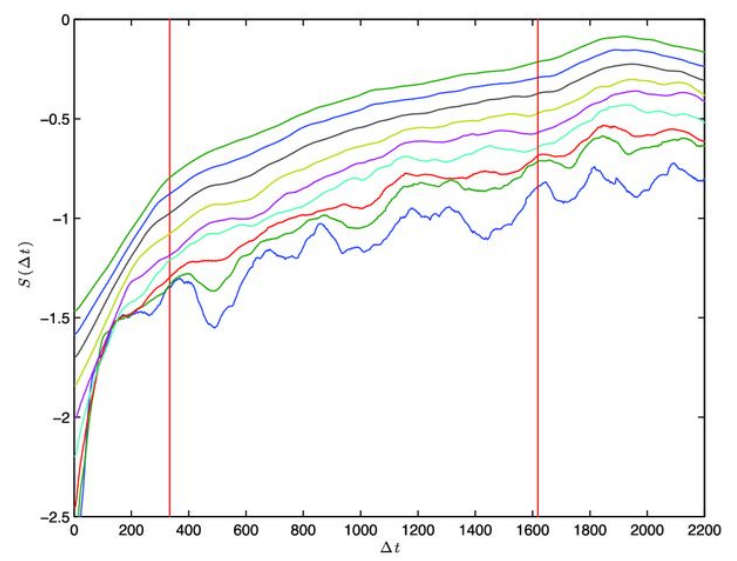

(a)

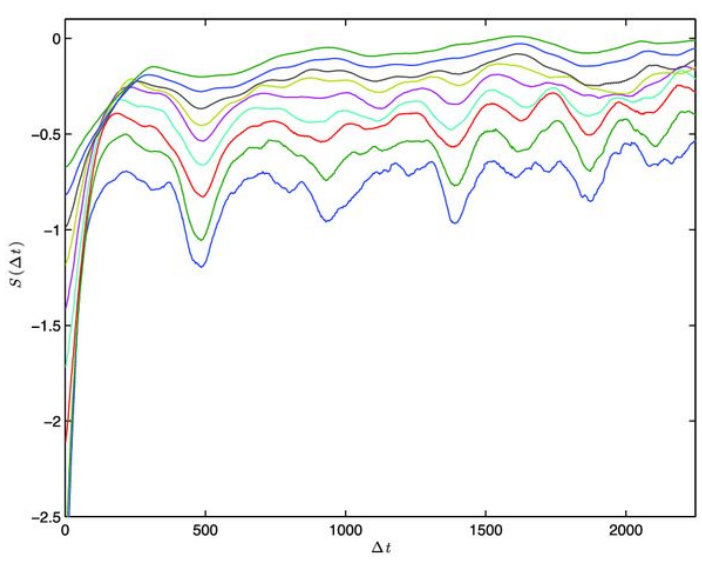

(b)

Figure 4.13: (a) $S(\Delta t)$ versus time elapsed for B1828-11. Each curve is for a different embedding dimension from 2-10 starting at the lowest curve. The vertical red lines mark the region where least square regression was applied. This corresponds to an average maximum Lyapunov exponent of $\lambda_{\max }=(4.0 \pm 0.3) \times 10^{-4}$ inverse days. (b) $S(\Delta t)$ for a surrogate data set for B1828-11.

Once the neighborhood has converged, we see a similar linear behavior across all the embedding dimensions. This continues until the curves start to saturate to a value on the scale of the attractor for that particular embedded dimension.

The positive slope of the linear region suggests that B1828-11 is a chaotic system with a maximum Lyapunov exponent of $(4.0 \pm 0.3) \times 10^{-4}$ inverse days. When the same procedure is done to a surrogate data set of B1828-11, no linear region is observed. Once the surrogate neighborhood passes the convergent region it directly saturates around a constant value. This constant saturation would suggest a Lyapunov exponent of zero, which is what is expected for a linear dynamical equation. The surrogate's strikingly different behavior would imply that the linear region in B1828-11 is a real detection and is not a consequence of a random process.

When this is applied to the other pulsars there are no other definitive detections. Either the convergent region is dominant causing a direct saturation, or the total 
time of the measurement is too small to positively state a separation between the convergent and linear regions. We will expand on these ideas more in the following section.

\subsubsection{Lyapunov Benchmark Testing}

Again we want to ensure that the algorithm is working in its entirety to produce the known values of the Lyapunov exponent. Therefore we follow the same procedure as Section 4.2.1.8, matching the same number of turning points and sampling spacing of the pulsar. Because B1828-11 is our only detection, we concentrate our attention on its benchmark results.

The Lyapunov exponent changes with different parameters in the governing equations. Because of this, we use the most common chaotic parameters for the Lorenz and Rössler attractors ${ }^{4}$. Under these conditions, the maximum Lyapunov exponent for the Lorenz attractor is $\lambda_{\max } \simeq 0.9056$ and for the Rössler is $\lambda_{\max } \simeq 0.0714$ (Sprott, 2003).

The results of the benchmark testing of B1828-11, seen in Fig. 4.14, seem to produce conflicting results. The algorithm estimates a maximum exponent for the Rössler attractor to be a reasonable $\lambda_{\max }=0.080 \pm 0.003$, but for the Lorenz attractor it estimates an unreasonable value of $\lambda_{\max }=0.28 \pm 0.05$.

The cause of this discrepancy can be seen in the surrogate results. There we can see that the convergence time-scales are affecting our estimates. For the Rössler attractor, the time for the neighborhood to converge is about 5 time units, while

\footnotetext{
${ }^{4}$ Lorenz: $\sigma=10, r=28, b=8 / 3 ;$ Rössler $: a=b=0.2, c=5.7$
} 

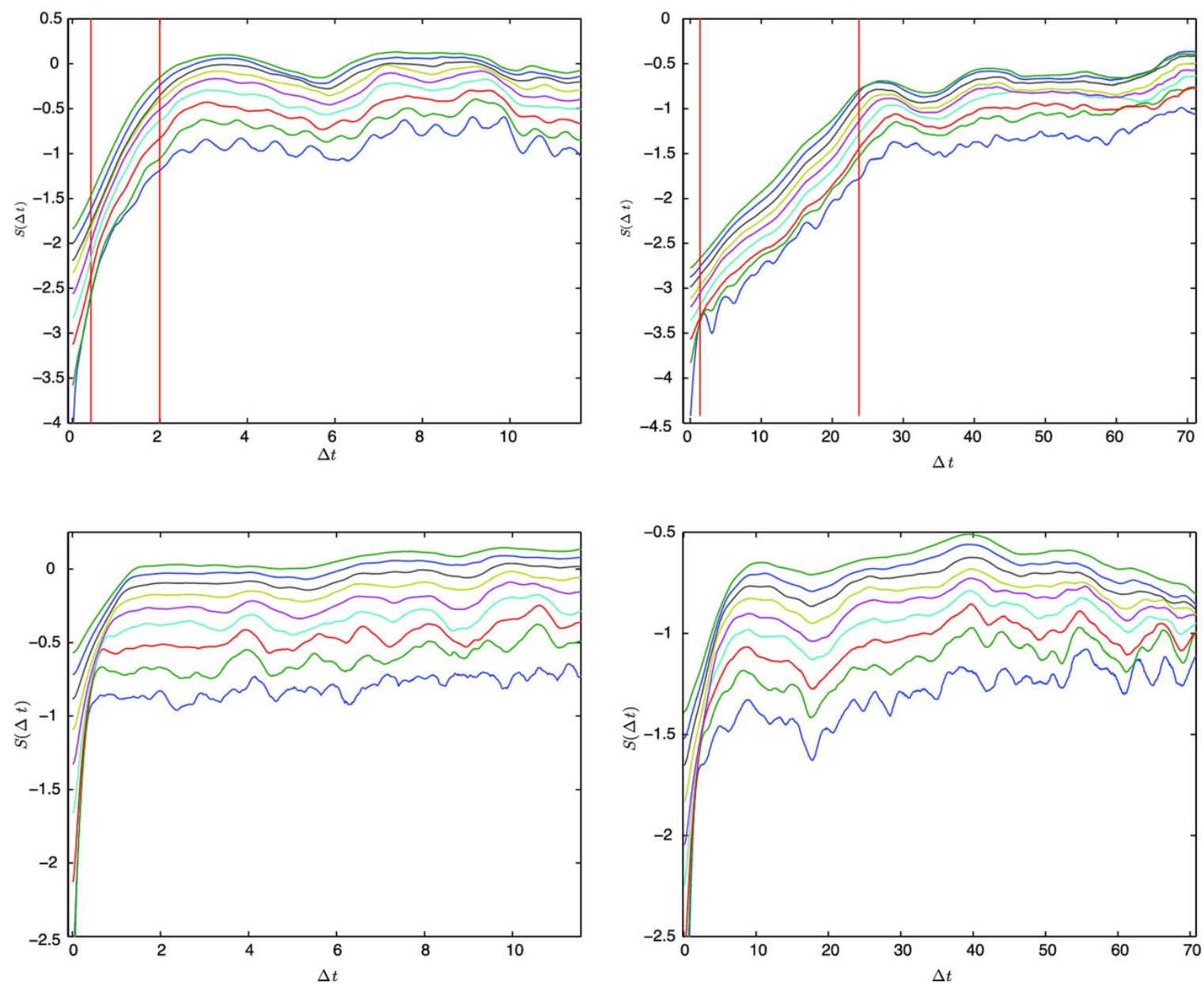

Figure 4.14: Benchmark results for B1828-11. Left column is the Lorenz attractor results. Right column is the Rössler attractor results. Top row are the $S(\Delta t)$ for reconstructed attractors. Bottom row are the $S(\Delta t)$ surrogate data for the appropriate attractors. 
the exponential behavior will last on time scales of $\frac{1}{\lambda_{\max }} \approx 14$ units. Therefore, the Rössler attractor is outlasting the convergence time and will be able to demonstrate its exponential behavior. The Lorenz attractor with these parameter values is more sensitive to initial conditions and will only exhibit its exponential behavior on a time-

scale of $\frac{1}{\lambda_{\max }} \approx 1$ unit, which is on the same scale of its convergence time. Therefore the $S(\Delta t)$ for the Lorenz attractor is not portraying its true behavior.

This convergence time is partly inherent to the system and partly due to noise. Because of this, there are two ways to correct this behavior. The first is to simply reduce the noise in the time series. The other approach is to increase the sampling period of the measurements in order to start with smaller neighborhoods which will give the exponential behavior several orders of magnitude to appear. Unfortunately, we are unable to comfortably remove any more noise in the algorithm. Also, to reduce the neighborhoods by orders of magnitude, we have to increase the number of data points by orders of magnitudes, which is currently beyond our system's capabilities.

Because of this convergence time, our algorithm is sensitive to lower levels of chaos with smaller Lyapunov exponents and is not able to distinguish between higher levels of chaos and the convergence region.

\subsection{Conclusions}

By using a careful combination of turning point analysis, cubic splining, and Fourier transforms, we have constructed an algorithm that re-samples an unevenly spaced time series without losing structural information. We have demonstrated this 
through an array of benchmark testing with known chaotic time series under similar conditions to a given pulsar time series. This testing has shown that there are not any significant changes in the correlation dimension or the maximum Lyapunov exponents, when it was detectable.

These techniques were applied to the pulsar spin-down rates from Lyne et al. (2010), see Appendix D, where B1828-11 exhibits clear chaotic behavior. We have shown that the measurements of its correlation dimension and maximum Lyapunov exponents are largely invariant across embedding dimensions. This, combined with its radically different reactions compared to its surrogate data sets, has shown that the chaotic characteristics in this pulsar are not caused by random processes.

The positive measurement of $\lambda_{\max }=(4.0 \pm 0.3) \times 10^{-4}$ inverse days confirms that B1828-11 is chaotic in nature. For a system of equations to be chaotic, there needs to be a minimum of three dynamical equations with three governing variables. The correlation dimension measurement of B1828-11, $D=2.06 \pm 0.03$, implies that there are a total of three governing variables, meeting the minimum requirements for chaos. One governing variable is clearly the spin-down rate of the pulsar. At this time, we can only speculate about the other two. We know that the magnetic fields of a pulsar can change its dynamics and change the pulse profile. It has further been shown that changes to the superfluid interior can affect the long term dynamics of a pulsar (Ho \& Andersson, 2012). Therefore, these seem to be excellent candidates. Beyond this, the subject is still a mystery and has to be explored with non-linear simulations. Regardless of the model chosen, it is possible to perform some of the 
methods presented here, on the simulated data, to see if similar chaotic behaviors are present.

Knowing that B1828-11 is governed by three variables, the reconstructed attractor in Fig. 4.7 would be an accurate depiction of its strange attractor. Because this is visually similar to the other attractors in Fig. 4.7, we would find it peculiar if their dynamics were not somehow related. Unfortunately, the techniques in this thesis were unable to confirm this relationship. If these pulsars continue to be observed with an increased cadence, this would improve the correlation dimension and Lyapunov exponent measurements, perhaps to the point where these similarities could be quantified. With these measurements and a working model, estimates for the parameters can be given, which will give us additional insight into the interior and/or exterior of these pulsars and how this relates to their dynamics. 


\section{Chapter 5}

\section{Conclusions}

This work has focused on the emission and spin dynamics variabilities in pulsars and the interaction between the two. While the results presented here have significantly contributed to the understanding this difficult question, there is still a great deal that is still obscure. Therefore, we recap the results from this thesis and discuss how future work will further improve the knowledge of this subject.

In Chapter 2, we have been able to reveal bursting events in B0611+22 at both $327 \mathrm{MHz}$ and $1400 \mathrm{MHz}$. Knowing that B0611+22 is a bursting pulsar and the changes to its profile may help more accurately predict the time of arrival of its pulses. Yet, these bursting events behave differently at these frequencies. These behaviors question whether the bursting events are an additional effect or in competing operation. In the future, we hope to have simultaneous observations at different frequencies to determine which operation is true and to see how a burst could be propagating through these different emission regions.

The bursting behaviors in $\mathrm{B} 0611+22$ are analogous to the radio behaviors seen in the mode switching pulsar B0943+10 but in reverse as seen in Fig. 5.1, where B0943+10 has a bright mode gradually increases in strength before declining rapidly to a radio-quiet state. A recent discovery has shown X-ray state switching for B0943+10, where non-thermal un-pulsed X-rays are observed during the bright 
radio emitting phases, while an additional pulse thermal component is present during the radio quiet phase (Hermsen et al., 2013). With a recently scheduled X-ray observation using the $X M M$-Newton telescope, we will shortly be investigating whether similar behaviors are seen in B0611+22.

Knowing that other bursting pulsars have a rather stable normal mode, we searched for such a relationship within the previously thought nulling events of J1752+2359 within Chapter 3 .

This search uncovered, with the aid of positive number statistics, a weak profile within these events. In the future, an analysis of a lengthier observation will more definitively prove the presence of this mode. Yet, this work shows that all the bursting pulsars to date are operating under similar conditions such that

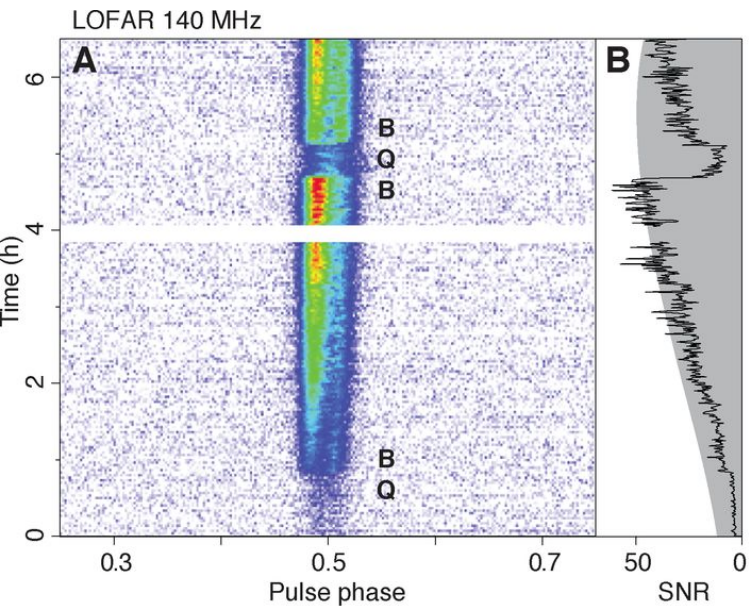

Figure 5.1: B0943+10 modes at $140 \mathrm{MHz}$ as presented in Hermsen et al. (2013). (A) Identification of the $\mathrm{B}$ and $\mathrm{Q}$ modes with LOFAR at $140 \mathrm{MHz}$, showing pulse intensity versus rotational phase and time. A 10-min section (at the 4-hour mark) contaminated by interference is blanked out. (B) Comparison of the measured signal-tonoise ratio (SNR) and the nominal relative LOFAR sensitivity, changing with elevation throughout the observation (gray scale), normalized over the 4.0 to 4.5 -hour both of these modes are being produced. range.

The nonlinear studies presented in Chapter 4 suggests the spin-down rate fluctuations in B1828-11 are chaotic in nature. This study also showed that these fluctuations are governed by three physical quantities with one being the spin-down rate itself. Also, the structure of other attractors reconstructed from the spin-down rates bear strong resemblance to B1828-11. While 


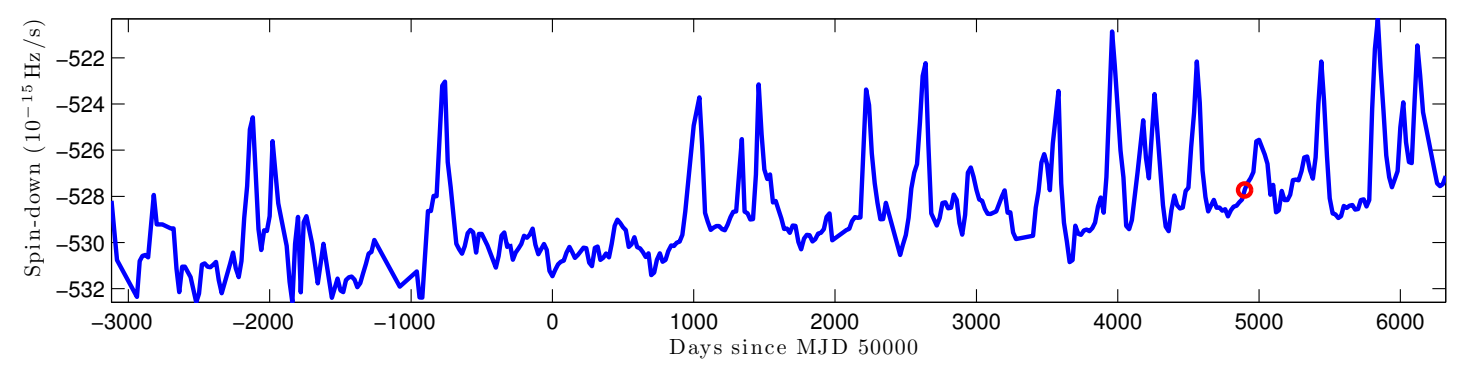

Figure 5.2: The spin down rate changes for B0611+22 supplied by Andrew Lyne. The red circle marks when the data in Chapter 2 was recorded.

this is not definitive, hints that other spin-down rate changes in pulsars are governed by the same physical mechanisms as B1828-11. As these and other pulsars are observed at higher cadence, this analysis will be more effective as these data sets cover even larger amounts of time. Also, as non-linear models are developed, these methods can be used to compare Lyapunov exponents and correlation dimension values with observed quantities.

The bursting phenomena seen in B0611+22 and J1752+2359 seem independent of the spin-down rate changes seen in B1828-11 and the other pulsars in Lyne et al. (2010), but data supplied by Andrew Lyne hints at another possibility. The spin-down rate changes recorded for B0611+22 over the last 25 years, shown in Fig. 5.2, show sharp increases in the frequency derivative. Since all of our observations are around a similar epoch, it is currently not possible to say how these two relate. Future observations will investigate whether a correlation is present. 


\section{Bibliography}

Argoul, F., Arneodo, A., Richetti, P., Roux, J. C., \& Swinney, H. L. 1987, Accounts of Chemical Research, 20, 436

Backer, D. C. 1970a, Nature, 228, 752

-. 1970b, Nature, 227, 692

—. 1970c, Nature, 228, 1297

—. 1970d, Nature, 228, 42

—. 1973, ApJ, 182, 245

Bell Burnell, S. J. 1977, Annals of the New York Academy of Sciences, 302, 685

Bhattacharya, D., \& van den Heuvel, E. P. J. 1991, Phys. Rep., 203, 1

Bombaci, I. 1996, A\&A, 305, 871

Brockwell, P. J., \& Davis, R. A. 1996, Time series: theory and methods, 2nd edn. (New York: Springer), 312,313

Brook, P. R., Karastergiou, A., Buchner, S., et al. 2014, ApJ, 780, L31

Chandler, A. M. 2003, PhD thesis, California Institute of Technology

Condon, J. J., \& Ransom, S. M. 2010, Essential Radio Astronomy

Cordes, J. M., \& Shannon, R. M. 2008, ApJ, 682, 1152

Davies, J. G., Lyne, A. G., \& Seiradakis, J. H. 1972, Nature, 240, 229

Deshpande, A. A., \& Rankin, J. M. 1999, ApJ, 524, 1008

Dowd, A., Sisk, W., \& Hagen, J. 2000, in Astronomical Society of the Pacific Conference Series, Vol. 202, IAU Colloq. 177: Pulsar Astronomy - 2000 and Beyond, ed. M. Kramer, N. Wex, \& R. Wielebinski, 275

Drake, F. D., \& Craft, H. D. 1968, Nature, 220, 231

Ferguson, D. C., \& Boriakoff, V. 1980, ApJ, 239, 310

Gold, T. 1968, NATURE, 218, 731

Goldreich, P., \& Julian, W. H. 1969, ApJ, 157, 869

Grassberger, P., \& Procaccia, I. 1983, Physica D: Nonlinear Phenomena, 9, 189

Hegger, R., Kantz, H., \& Schreiber, T. 1999, Chaos: An Interdisciplinary Journal of Nonlinear Science, 9, 413 
Helfand, D. J., Taylor, J. H., Backus, P. R., \& Cordes, J. M. 1980, ApJ, 237, 206

Hermsen, W., Hessels, J. W. T., Kuiper, L., et al. 2013, Science, 339, 436

Hewish, A., Bell, S. J., Pilkington, J. D. H., Scott, P. F., \& Collins, R. A. 1968, Nature, 217, 709

Ho, W. C. G., \& Andersson, N. 2012, Nat Phys, advance online publication,

Hobbs, G., Lyne, A., \& Kramer, M. 2006, Chinese Journal of Astronomy and Astrophysics, 6, 169

Jackson, J. D. 1962, Classical electrodynamics (New York: Wiley)

Jones, D. I. 2012, Monthly Notices of the Royal Astronomical Society, 420, 2325

Kantz, H. 1994, Physics Letters A, 185, 77

Kantz, H., \& Schreiber, T. 2004, Nonlinear time series analysis, 2nd edn. (Cambridge, UK: Cambridge University Press)

Kendall, M. G., \& Stuart, A. 1966, The advanced theory of statistics, Vol. 3 (London: C. Griffin), 351,352

Kramer, M., Lyne, A. G., O'Brien, J. T., Jordan, C. A., \& Lorimer, D. R. 2006, Science, 312, 549

Large, M. I., Vaughan, A. E., \& Mills, B. Y. 1968, Nature, 220, 340

Lewandowski, W., Wolszczan, A., Feiler, G., Konacki, M., \& Sołtysiński, T. 2004, ApJ, 600, 905

Lorenz, E. N. 1963, Journal of the Atmospheric Sciences, 20, 130

—. 1993, The essence of chaos (Seattle: University of Washington Press)

Lorimer, D. 2011, AIP Conf.Proc., 1357, 11

Lorimer, D. R., Camilo, F., \& McLaughlin, M. A. 2013, MNRAS, submitted

Lorimer, D. R., \& Kramer, M. 2005, Handbook of pulsar astronomy, Vol. 4 (Cambridge, UK: Cambridge University Press)

Lyne, A., Hobbs, G., Kramer, M., Stairs, I., \& Stappers, B. 2010, Science, 329, 408

Lyne, A. G., \& Graham-Smith, F. 2012, Cambridge astrophysics, Vol. 48, Pulsar astronomy, 4th edn. (Cambridge: Cambridge University Press)

Maan, Y., Deshpande, A. A., Chandrashekar, V., et al. 2013, The Astrophysical Journal Supplement Series, 204, 12

Manchester, R. N., Hobbs, G. B., Teoh, A., \& Hobbs, M. 2005, AJ, 129, 1993 
Manchester, R. N., \& Taylor, J. H. 1977, Pulsars (San Francisco: W. H. Freeman)

Mathieu, J., \& Scott, J. 2000, An introduction to turbulent flow (Cambridge: Cambridge University Press)

Mattson, B., Gibb, M., \& Newman, P. 2010, Neutron Stars and Pulsars

McLaughlin, M. A., Lyne, A. G., Lorimer, D. R., et al. 2006, Nature, 439, 817

Michel, F. C., \& Li, H. 1999, Phys. Rep., 318, 227

Nowakowski, L. A. 1992, in IAU Colloq. 128: Magnetospheric Structure and Emission Mechanics of Radio Pulsars, ed. T. H. Hankins, J. M. Rankin, \& J. A. Gil, 280

Nowakowski, L. A., \& Rivera, N. S. 2000, in Bulletin of the American Astronomical Society, Vol. 33, American Astronomical Society Meeting Abstracts, 130.04

Olsen, L. F., \& Degn, H. 1985, Quarterly Reviews of Biophysics, 18, 165

Oppenheimer, J. R., \& Volkoff, G. M. 1939, Physical Review, 55, 374

Ostriker, J. P., \& Gunn, J. E. 1969, ApJ, 157, 1395

Petit, G., \& Tavella, P. 1996, Astronomy and Astrophysics, 308, 290

Press, W. H. 2007, Numerical recipes: the art of scientific computing, 3rd edn. (Cambridge, UK: Cambridge University Press)

Provenzale, A., Smith, L., Vio, R., \& Murante, G. 1992, Physica D: Nonlinear Phenomena, 58, 31

Rankin, J. M., Stinebring, D. R., \& Weisberg, J. M. 1989, ApJ, 346, 869

Rosen, R., McLaughlin, M. A., \& Thompson, S. E. 2011, The Astrophysical Journal Letters, 728, L19

Rosenstein, M. T., Collins, J. J., \& Luca, C. J. D. 1993, Physica D: Nonlinear Phenomena, 65, 117

Rössler, O. 1976, Physics Letters A, 57, 397

Ruderman, M. A., \& Sutherland, P. G. 1975, ApJ, 196, 51

Sauer, T., Yorke, J. A., \& Casdagli, M. 1991, Journal of Statistical Physics, 65, 579

Scargle, J. D. 1992, in Statistical Challenges in Modern Astronomy, ed. E. D. Feigelson \&amp; G. J. Babu, 411-436

Schreiber, T., \& Schmitz, A. 1996, Phys. Rev. Lett., 77, 635

Serylak, M., Stappers, B. W., \& Weltevrede, P. 2009, A\&A, 506, 865 
Seymour, A. D., \& Lorimer, D. R. 2013, MNRAS, 428, 983

Seymour, A. D., Lorimer, D. R., \& Ridley, J. P. 2014, MNRAS, 439, 3951

Shannon, R., Cordes, J., Lazio, J., Kramer, M., \& Lyne, A. 2009, in Bulletin of the American Astronomical Society, Vol. 41, American Astronomical Society Meeting Abstracts \#213, 436.06

Shklovskii, I. S. 1969, Astron. Zhurnal, 46, 715

Smale, S. 1967, Bull. Am. Math. Soc., 73, 747

Smith, F. G. 1973, MNRAS, 161, 9P

Sprott, J. C. 2003, Chaos and time-series analysis (Oxford: Oxford University Press)

Staelin, D. H., \& Reifenstein, III, E. C. 1968, Science, 162, 1481

Strogatz, S. H. 1994, Nonlinear dynamics and Chaos: with applications to physics, biology, chemistry, and engineering (Reading, Mass.: Addison-Wesley Pub.)

Sturrock, P. A. 1971, ApJ, 164, 529

Takens, F. 1981, Detecting strange attractors in turbulence

Theiler, J. 1986, Phys. Rev. A; Physical Review A, 34, 2427

Theiler, J., Eubank, S., Longtin, A., Galdrikian, B., \& Farmer, J. D. 1992, Physica D: Nonlinear Phenomena, 58, 77

Wang, N., Manchester, R. N., \& Johnston, S. 2007, MNRAS, 377, 1383

Weisberg, J. M., Cordes, J. M., Lundgren, S. C., et al. 1999, ApJS, 121, 171

Weltevrede, P., Edwards, R. T., \& Stappers, B. W. 2006, A\&A, 445, 243

Weltevrede, P., Johnston, S., \& Espinoza, C. M. 2011, MNRAS, 411, 1917

Weltevrede, P., Stappers, B. W., \& Edwards, R. T. 2007, A\&A, 469, 607 


\section{Appendix A}

\section{Skewness equations}

Here we describe the method used in Chapter 2 to calculate the skewness measurement of a pulse. Skewness is used to measure the asymmetry of a probability distribution and not geometric shapes. Therefore, we need to adapt its definition for our purpose.

Skewness is defined as

$$
\mathcal{S} \equiv \mathrm{E}\left[\left(\frac{x-\mathrm{E}[x]}{\operatorname{std}[x]}\right)^{3}\right]
$$

where $x$ is a bin value, $\mathrm{E}[x]$ is the mean value of $x$ and $\operatorname{std}[x]$ is its standard deviation.

In Chapter 2 we treat each local mean pulse as a distribution function and use the flux densities $\left(y_{x}\right)$ for a weighted average. We define our weighting $\left(w_{x}\right)$ as

$$
w_{x} \equiv \frac{y_{x}}{\sum y_{x}}
$$

This then produces a weighted-mean value of

$$
\mathrm{E}\left[f_{x}\right]=\sum f_{x} w_{x}
$$


Here $f_{x}$ is a function of $x$. Using this definition we write the standard deviation as

$$
\operatorname{std}\left[f_{x}\right]=\sqrt{\mathrm{E}\left[f_{x}^{2}\right]-\mathrm{E}\left[f_{x}\right]^{2}}
$$

The skewness is then calculated with an expansion of its definition, i.e.

$$
\mathcal{S}=\frac{\mathrm{E}\left[x^{3}\right]-3 \mathrm{E}[x] \operatorname{std}[x]^{2}-\mathrm{E}[x]^{3}}{\operatorname{std}[x]^{3}}
$$




\section{Appendix B}

\section{Turning point variance derivation}

Here we present the derivation from Kendall \& Stuart (1966) for the turning point analysis, which was used in Chapter 4 to rank how adequately different data sets were sampled.

The number of turing points $p$ can be seen as a summation

$$
p=\sum_{i=1}^{n-2} X_{i}
$$

where $X_{i}$ is equal to one if $u_{i+1}$ is a turning point, and zero if not. We have already shown that the expectation value of the sum

$$
E(p)=\sum E\left(X_{i}\right)=\frac{2}{3}(n-2)
$$

In order to calculate the variance we need to find the expectation of the square of the number of turning points

$$
E\left(p^{2}\right)=E\left\{\left(\sum_{1}^{n-2} X_{i}\right)^{2}\right\}
$$


This can be expanded to

$$
\begin{aligned}
E\left(p^{2}\right)=E\left\{\sum_{n-2} X_{i}^{2}+2 \sum_{n-3} X_{i} X_{i+1}+2 \sum_{n-4}\right. & X_{i} X_{i+2} \\
& \left.+\sum_{(n-4)(n-5)} X_{i} X_{i+k}\right\}, k \neq 0,1,2,
\end{aligned}
$$

where the suffixes to the $\sum$ signs indicate the number of terms over which summation takes place. We then have

$$
\begin{aligned}
E\left(p^{2}\right)=(n-2) E\left(X_{i}^{2}\right)+2(n-3) E\left(X_{i} X_{i+1}\right)+ & 2(n-4) E\left(X_{i} X_{i+2}\right) \\
& +(n-4)(n-5) E\left(X_{i} X_{i+k}\right) .
\end{aligned}
$$

Since $X_{i}^{2}=X_{i}$, we have

$$
E\left(X_{i}^{2}\right)=\frac{2}{3}
$$

For $k>2, X_{i}$ and $X_{i+k}$ are independent, for they have no values in common. Thus

$$
E\left(X_{i} X_{i+k}\right)=E\left(X_{i}\right) E\left(X_{i+k}\right)=\frac{4}{9}
$$

We still need to evaluate $E\left(X_{i} X_{i+1}\right)$ and $E\left(X_{i} X_{i+2}\right)$. For the first term to contribute, there needs to be two consecutive turning points. In order to do this, we need a minimum of four data points, and define their values as $u_{1}<u_{2}<u_{3}<u_{4}$. This will generate 4 ! or 24 permutations, but one would find that only 10 permutations will contain two turning points. This leads to an expectation value of 


$$
E\left(X_{i} X_{i+1}\right)=\frac{10}{24}=\frac{5}{12} .
$$

Similarly, for $E\left(X_{i} X_{i+2}\right)$ five data points with five different values are needed. This produces 5 ! or 120 permutations, but only 54 will produce a turning point in the second and fourth locations. This leads us to

$$
E\left(X_{i} X_{i+2}\right)=\frac{54}{120}=\frac{9}{20} .
$$

Now, substituting these values into B.5 we find

$$
\begin{aligned}
& E\left(p^{2}\right)=\frac{2}{3}(n-2)+\frac{5}{6}(n-3)+\frac{9}{10}(n-4)+\frac{4}{9}(n-4)(n-5) \\
& E\left(p^{2}\right)=\frac{40 n^{2}-144 n+131}{90} .
\end{aligned}
$$

Finally, using the definition of the variance

$$
\sigma_{p}^{2}=E\left(p^{2}\right)-E(p)^{2},
$$

we insert our results from Equation B.2 and B.10 to conclude that

$$
\begin{aligned}
\sigma_{T}^{2} & =\frac{40 n^{2}-144 n+131}{90}-\frac{4(n-2)^{2}}{9} \\
\sigma_{T}^{2} & =\frac{16 n-29}{90} .
\end{aligned}
$$




\section{Appendix C}

\section{Correlation dimension error calculations}

The correlation dimension is the most common way to measure the fractal dimension of an attractor. Yet, the error calculations are not always well documented. Therefore, we present our method that was used to calculate the error on the correlation dimension measurements in Chapter 4.

The correlation sum, $C_{i}$, is the average of the pointwise measurements around the attractor for a certain test radius, $R_{i}$. Because of this, we can use the standard deviation of a mean as the uncertainty of the correlation sum for that particular radius,

$$
\sigma_{C_{i}}=\frac{\sigma_{\text {pointwise }}}{\sqrt{N-w}}
$$

where $N$ is the number of location vectors and $w$ is the Theiler window.

Next we use standard propagation of error techniques, to form the first order estimate of the uncertainty for the natural logarithm of the correlation sum,

$$
\sigma_{\ln C_{i}}=\frac{\sigma_{C_{i}}}{C_{i}}
$$

Within the desired scaling region, we follow the outline in Press (2007) for linear regression of least-squares to estimate the correlation dimension and its uncertainty. Press (2007) breaks this calculation into several summations. We have rewritten these sums for this application as follows: 


$$
\begin{gathered}
S \equiv \sum_{i=N_{0}}^{N_{C}} \frac{1}{\sigma_{\ln C_{i}}^{2}}, \\
S_{\ln R} \equiv \sum_{i=N_{0}}^{N_{C}} \frac{\ln R_{i}}{\sigma_{\ln C_{i}}^{2}} \\
S_{\ln C} \equiv \sum_{i=N_{0}}^{N_{C}} \frac{\ln C_{i}}{\sigma_{\ln C_{i}}^{2}} \\
S_{\ln R^{2}} \equiv \sum_{i=N_{0}}^{N_{C}} \frac{\left(\ln R_{i}\right)^{2}}{\sigma_{\ln C_{i}}^{2}}
\end{gathered}
$$

and

$$
S_{\ln R \ln C} \equiv \sum_{i=N_{0}}^{N_{C}} \frac{\ln R_{i} \ln C_{i}}{\sigma_{\ln C_{i}}^{2}}
$$

where $N_{0}$ and $N_{C}$ is the minimum and maximum index of the correlation sum measurements for a particular embedding dimension within the desired scaling region. With these summations, we are then able to calculate the slope. A common denominator value,

$$
\Delta \equiv S S_{\ln R^{2}}-\left(S_{\ln R}\right)^{2}
$$

is used to calculate the correlation dimension,

$$
d_{m}=\frac{S S_{\ln R \ln C}-S_{\ln R} S_{\ln C}}{\Delta}
$$


and variance,

$$
\sigma_{d_{m}}^{2}=\frac{S}{\Delta}
$$

for each embedding dimension.

To average the correlation dimension across the embedding dimensions, we use a weighted mean, where each measurement is weighted based on the inverse variance. For simplicity, we use normalized weighting coefficients,

$$
w_{m}=\frac{\sigma_{d_{m}}^{-2}}{\sum_{m=3}^{10} \sigma_{d_{m}}^{-2}} \text {. }
$$

With this weighting, the mean correlation dimension,

$$
<d>=\sum_{m=3}^{10} w_{m} d_{m}
$$

and the variance,

$$
\sigma_{<d>}^{2}=\sum_{m=3}^{10} w_{m}\left(d_{m}-<d>\right)^{2}
$$

are calculated. 


\section{Appendix D}

\section{Chaos figures}

Here we have tabulated all the figures generated during the nonlinear analysis

in Chapter 4 of the spin-down rate changes from Lyne et al. (2010).

Table D.1: A table of Fourier amplitudes and low pass time series for the non-linear analysis in Chapter 4. (Left) is the name of the pulsars in order of turning-point significance. (Middle) is the corresponding Fourier amplitudes versus normalized frequency of the longest section of 'well sampled data' form the spin-down rate time series. The vertical red line mark where the low pass filter was set based on turning point analysis. (Right upper) shows the normalized longest section of 'well sampled data' in red with the Fourier 'fit' in blue versus normalized time. (Right lower) is the difference between these two curves.

Pulsar Name




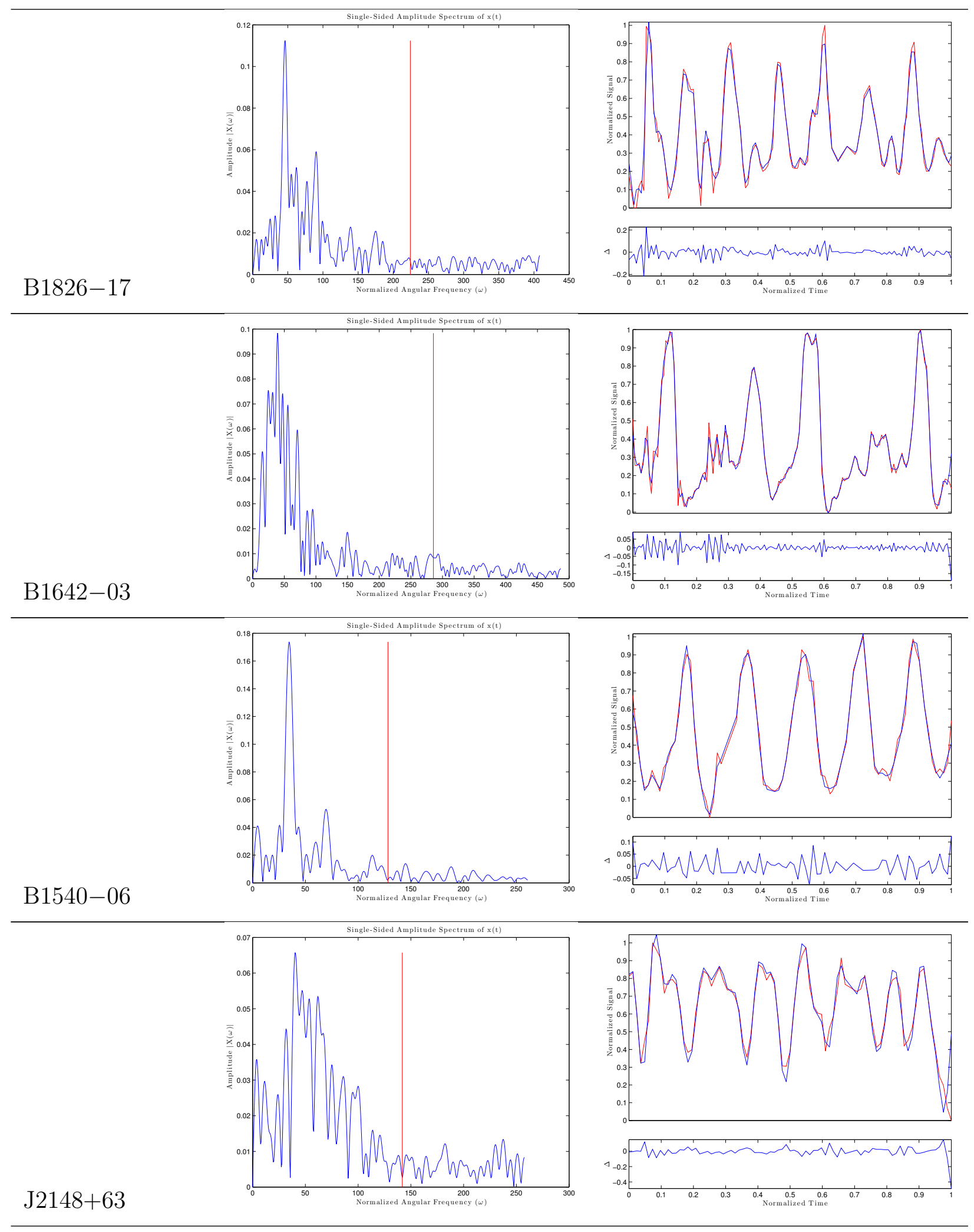




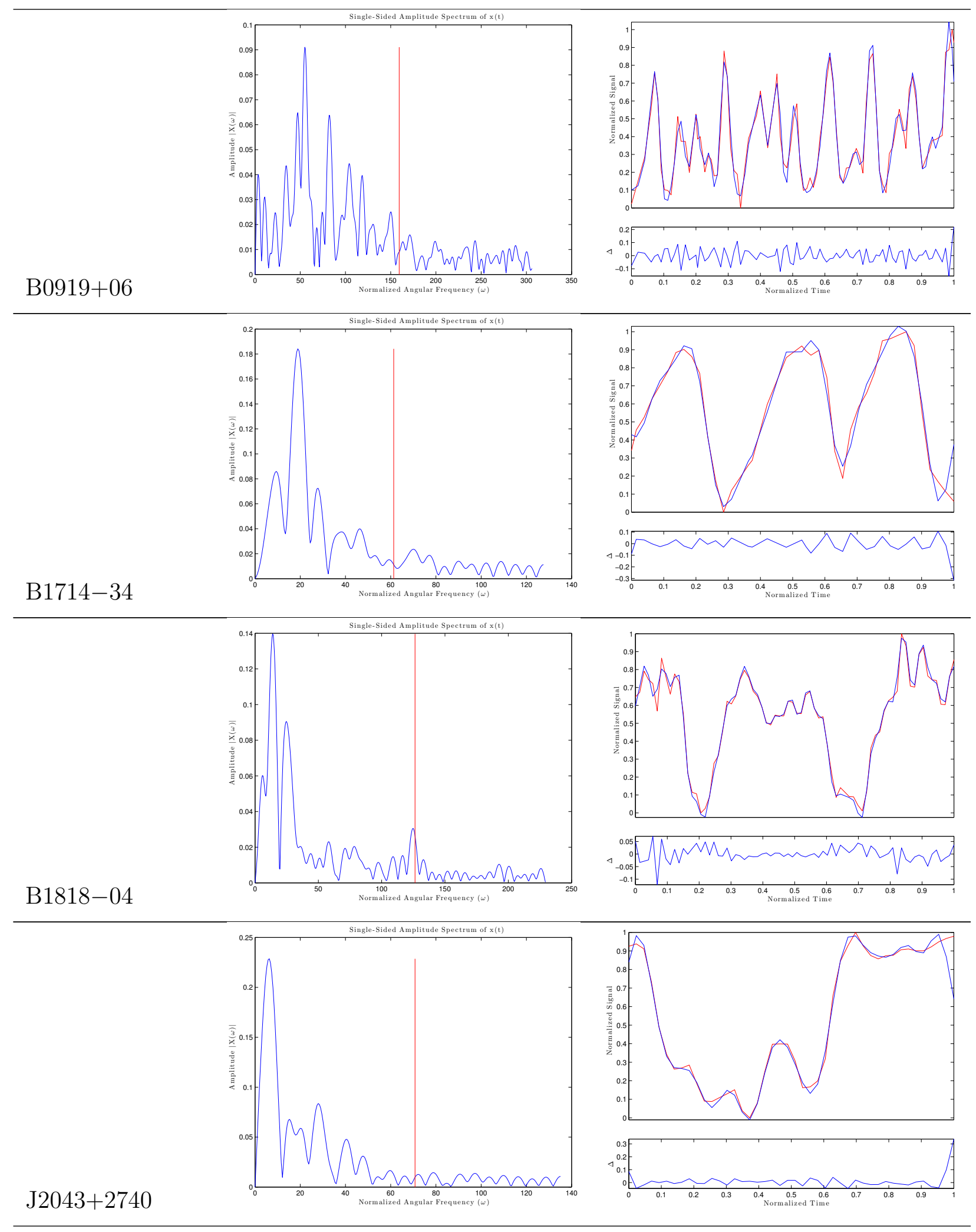




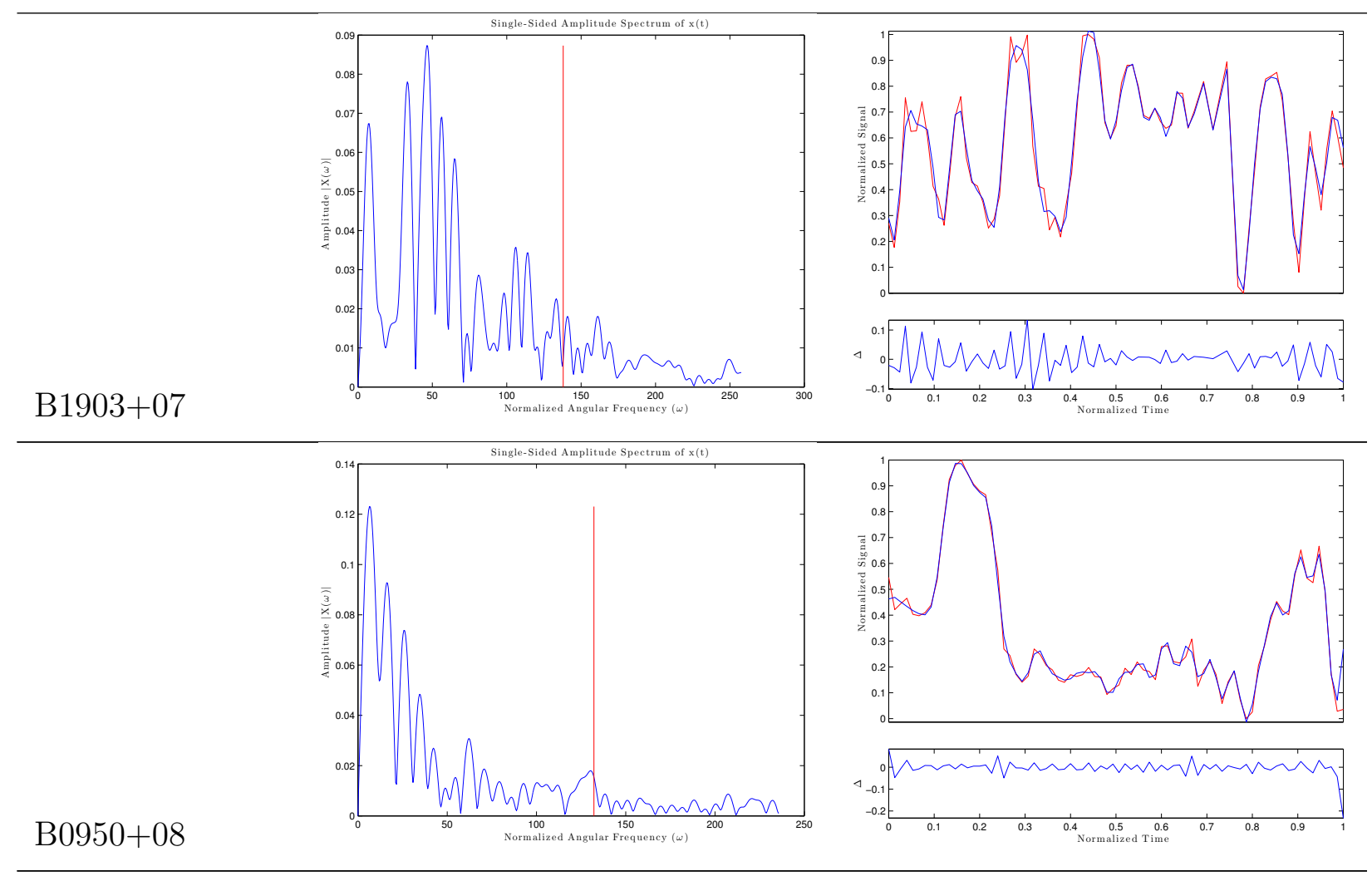

Table D.2: Reconstructed attractors using the method of delays with the data presented in Lyne et al. (2010), in order of turning point significance. The first three figures on the left show the attractors projection on the $(x(t), x(t+\tau))$, $(x(t+\tau), x(t+2 \tau))$, and $(x(t), x(t+2 \tau))$ coordinate planes. The last figure on the right shows the attractor in 3-D space, $(x(t), x(t+\tau), x(t+2 \tau))$. In each case $\tau$ was determined by the autocorrelation, see Chapter 4 for details.

Pulsar Name

Reconstructed attractors by the use of time delays
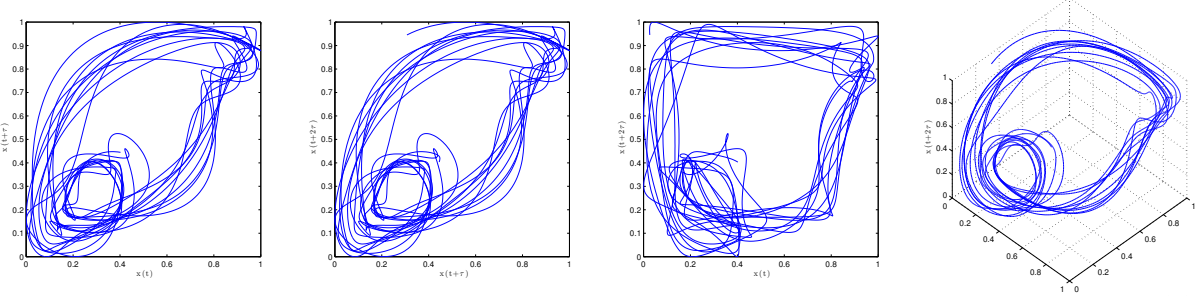

B1828-11 


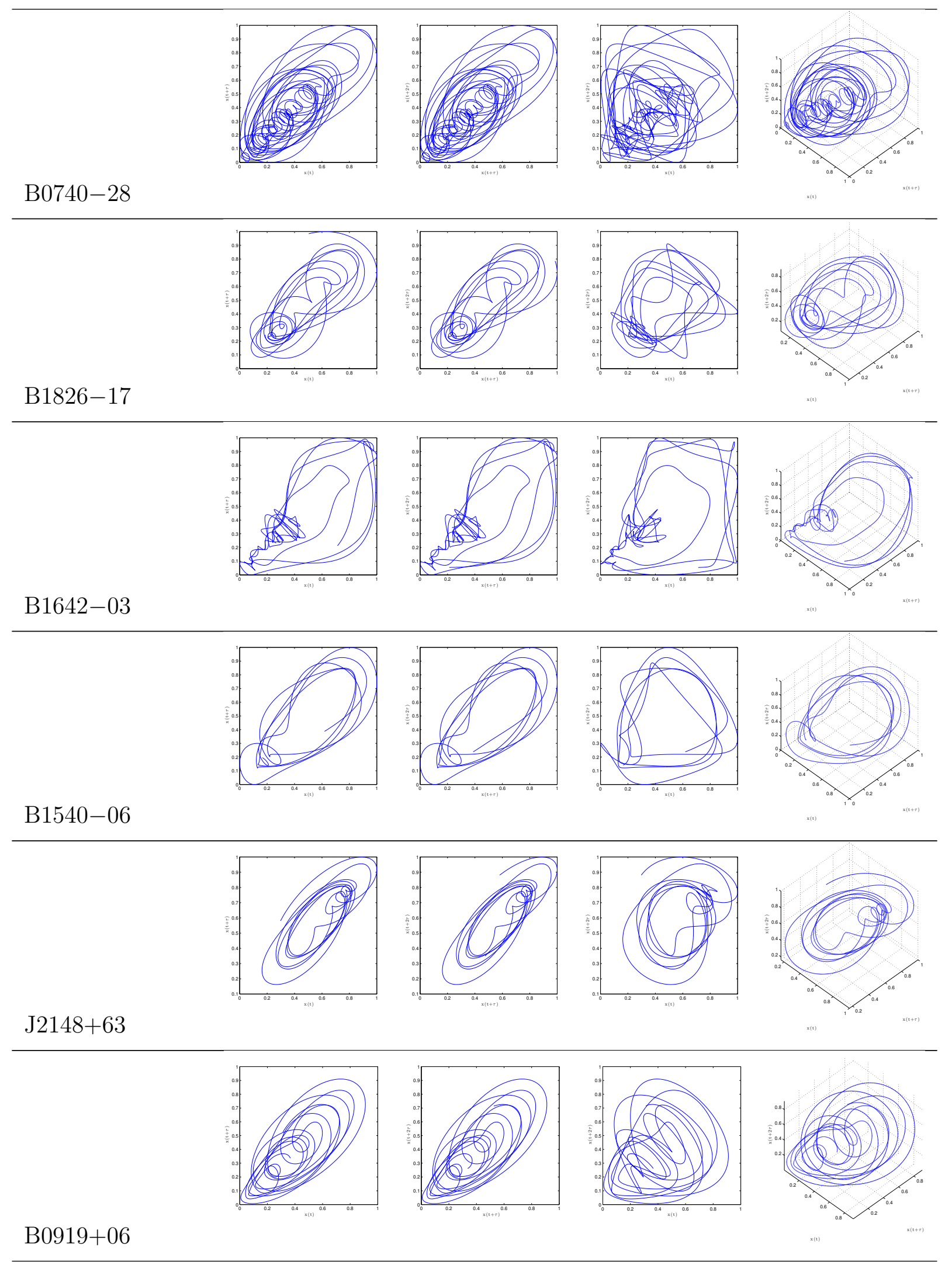




\section{B1714-34}
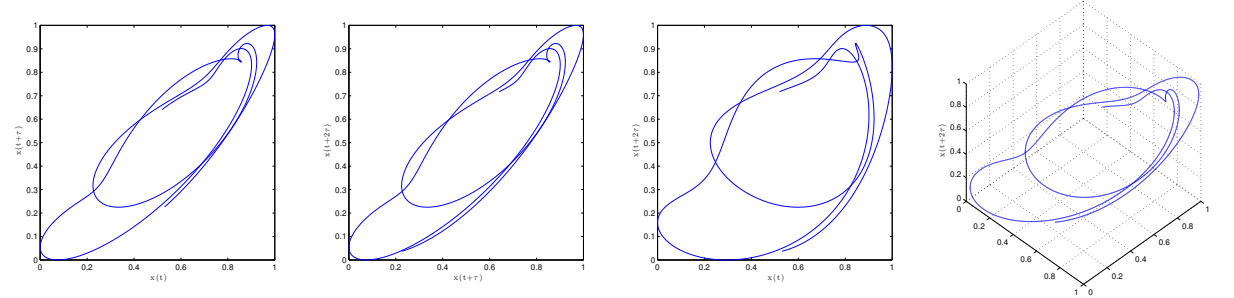

B1818-04
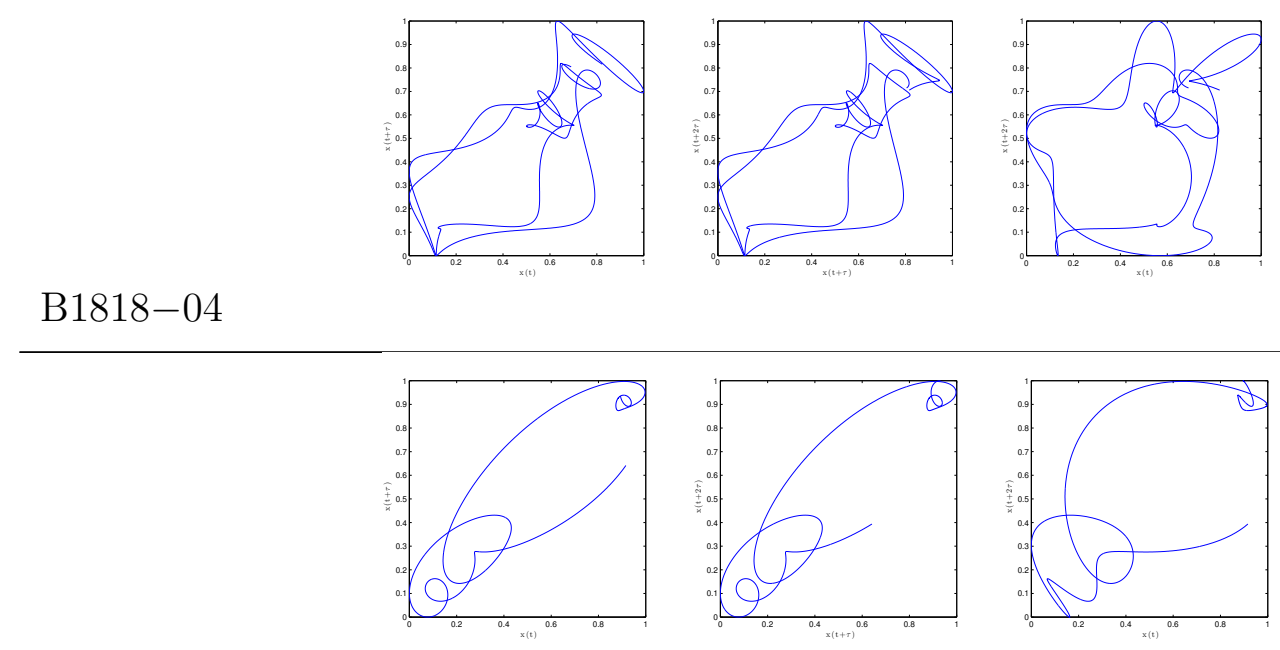

$x^{(t)}$

$\mathrm{J} 2043+2740$
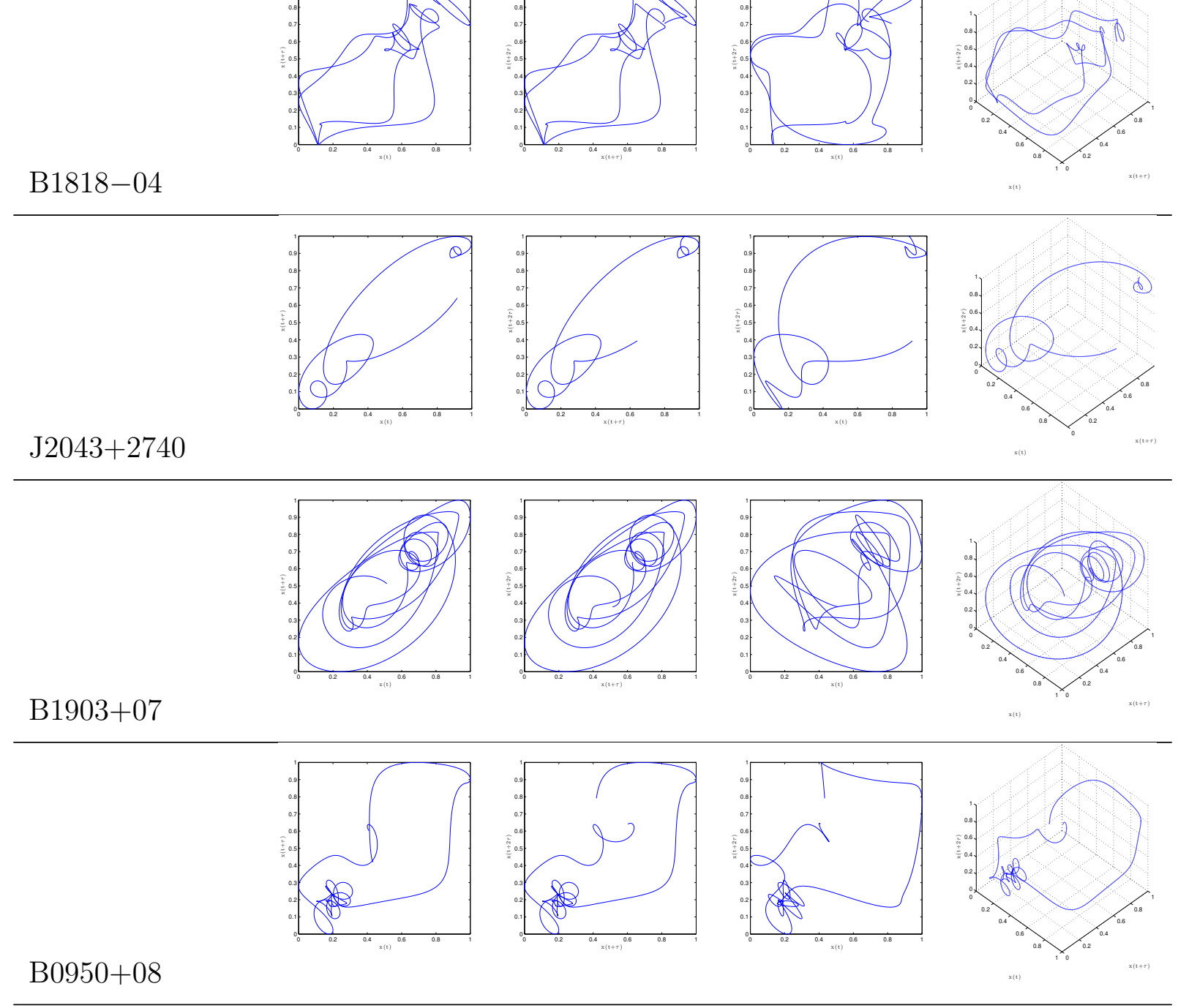
Table D.3: (Right) $\ln C$ versus $\ln R$ for the corresponding pulsar. The dashed horizontal blue lines mark the rough scaling region, $C=\frac{10}{n_{s}}$ to 0.10 . The solid horizontal red lines mark the flattest scaling region that is larger than a third of the rough scaling region. (Left) The flat blue line is the correlation dimension measurements, within the flattest scaling region, versus embedding dimension. The line at $45^{\circ}$ is what a purely random time series would be in that given $m$. The sloped red line is the mean and standard deviation of 10 surrogate data sets (see Chapter 4 ).

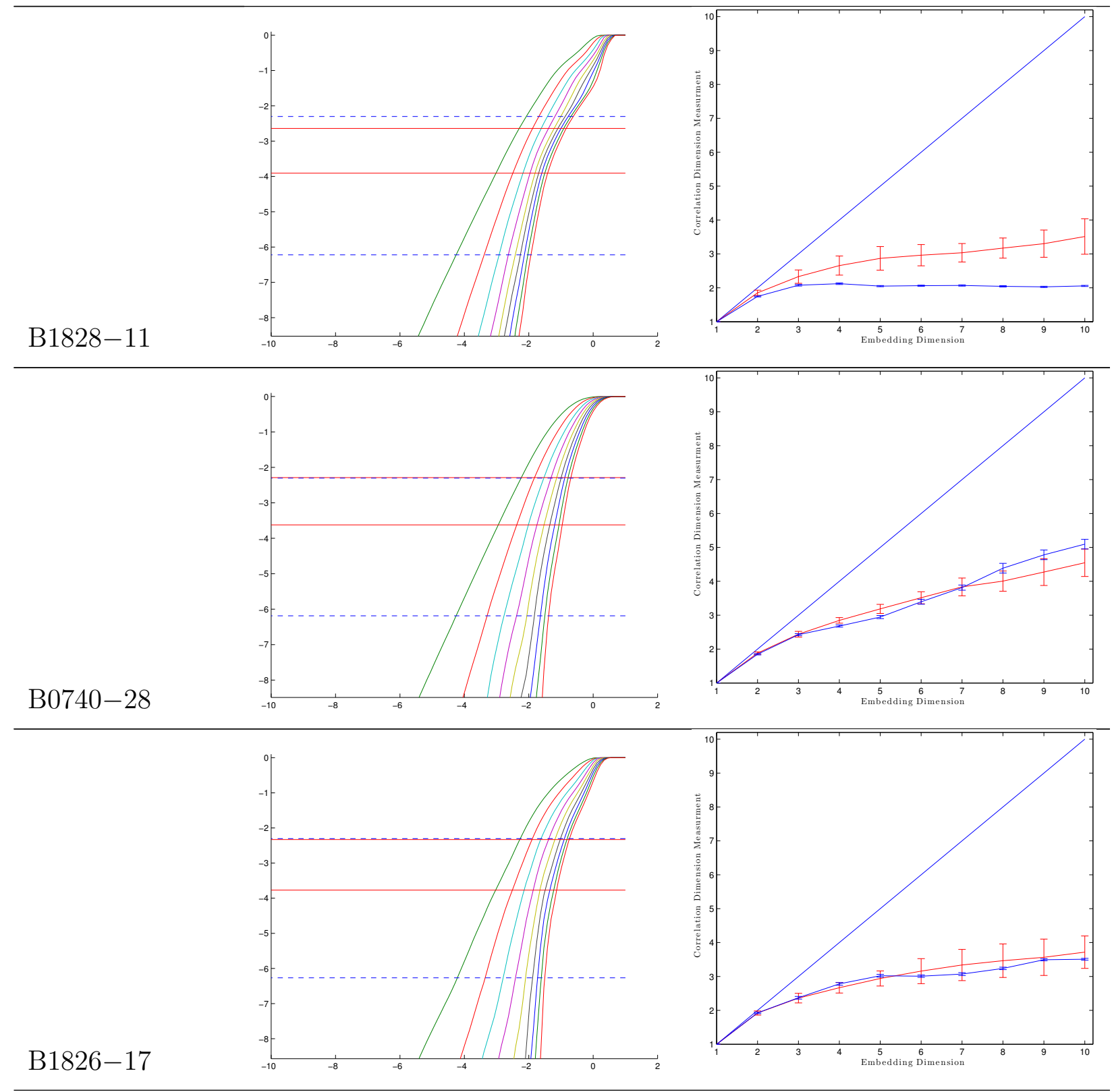




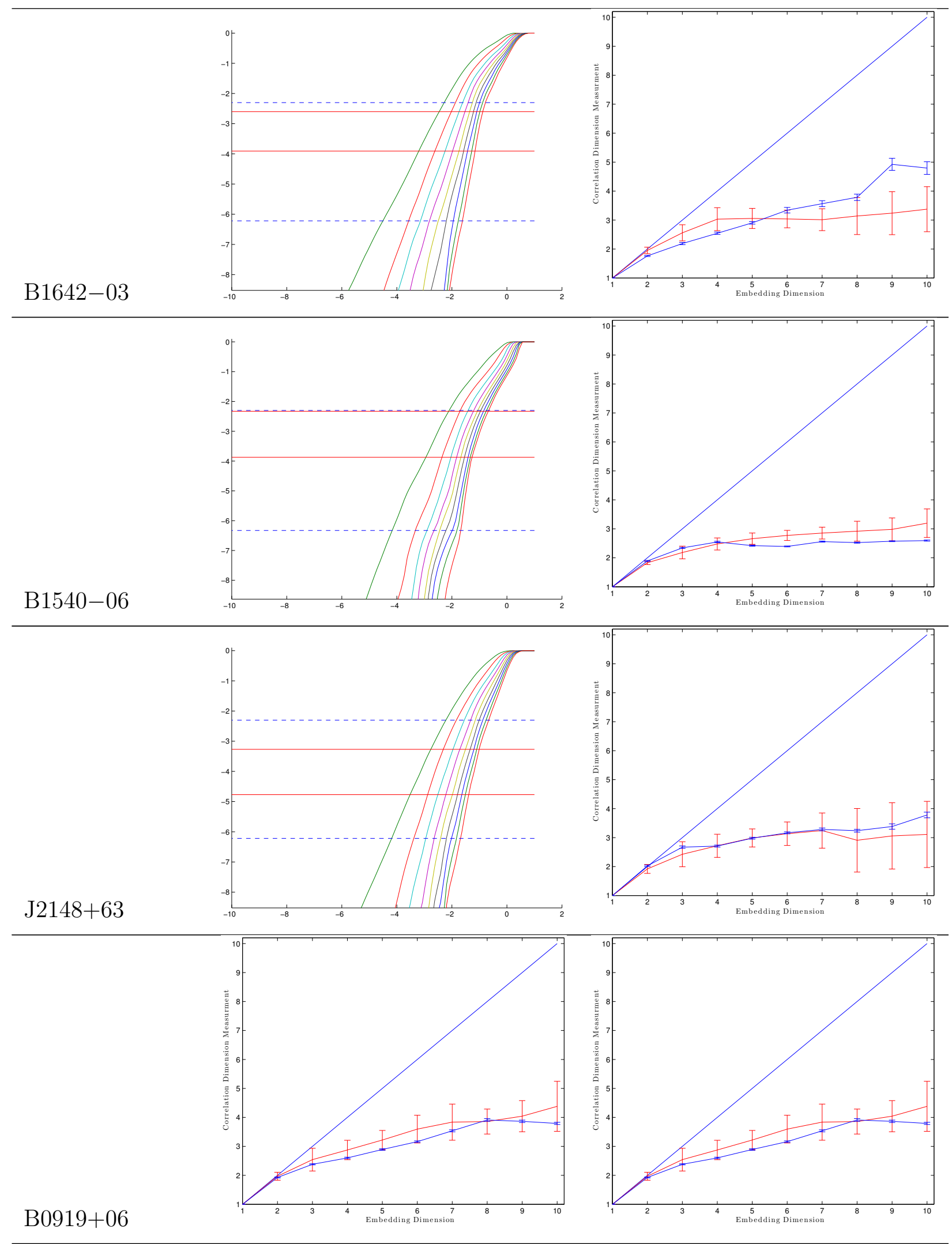




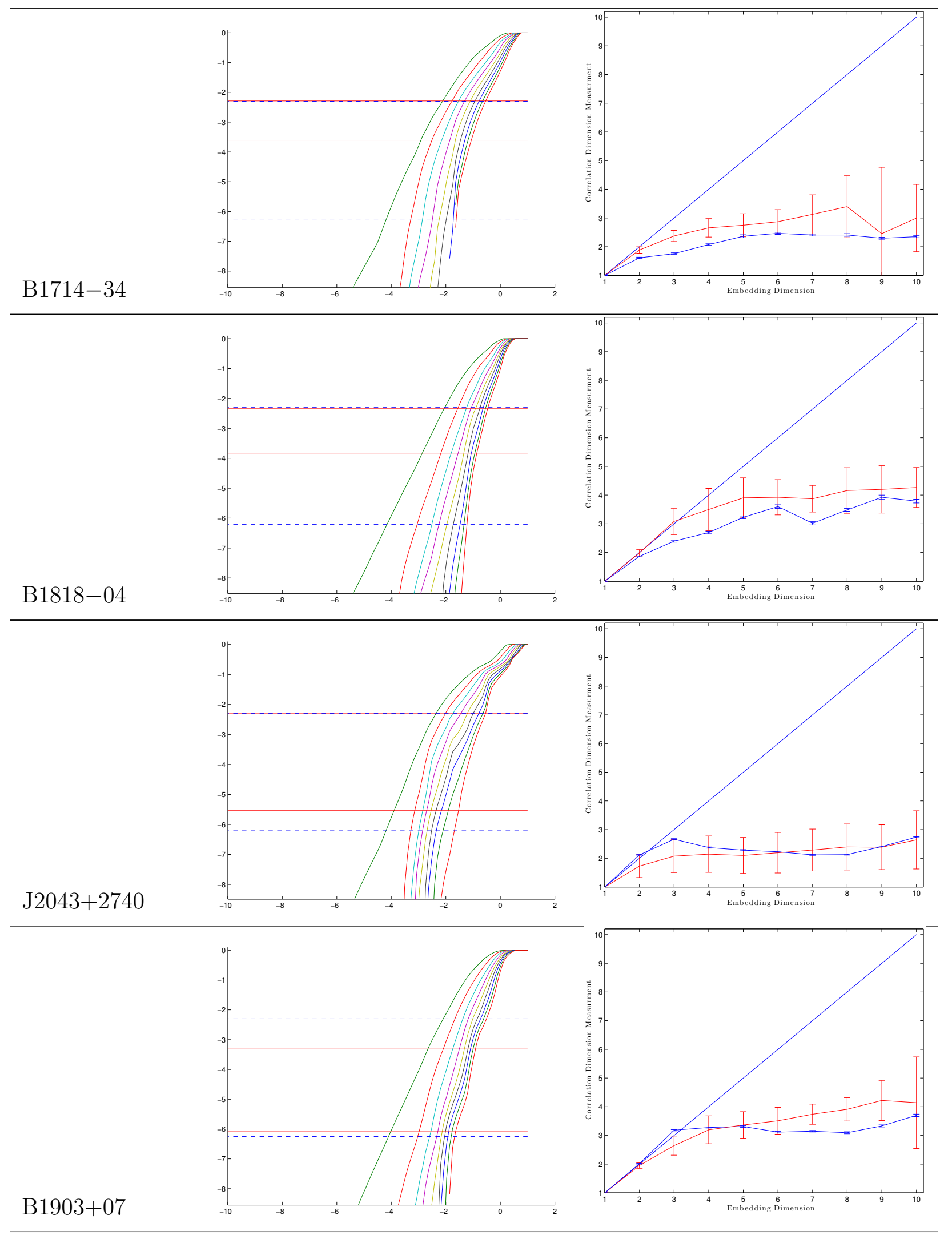




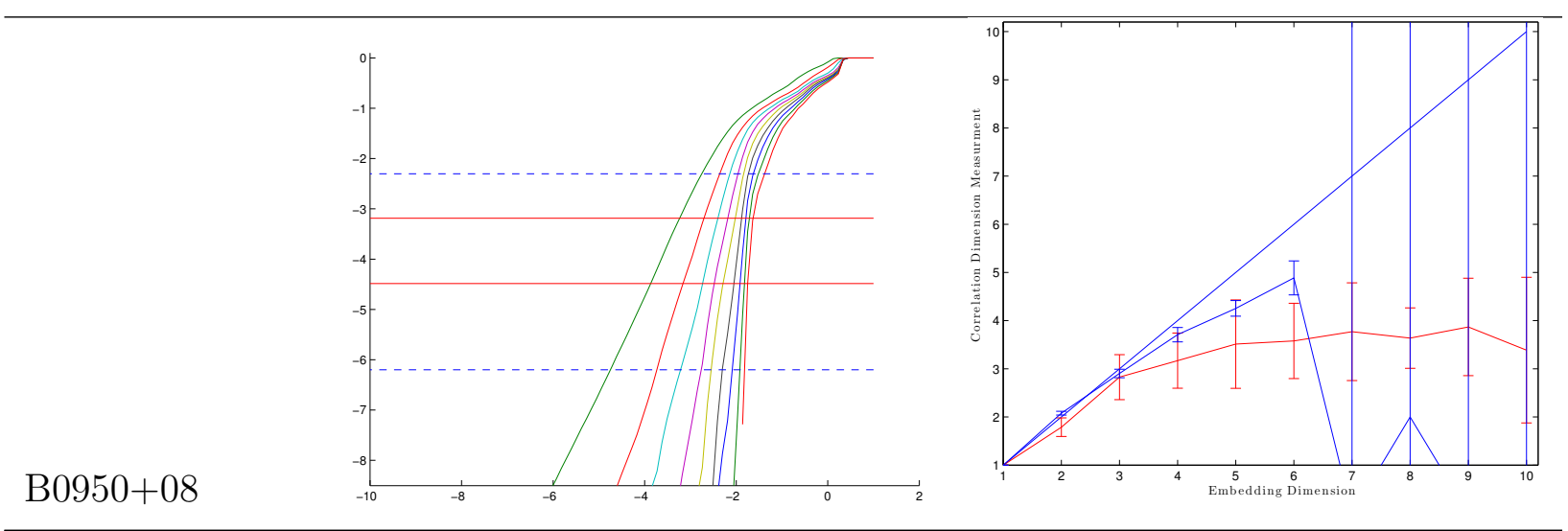

